\title{
A Letter of Intent for a Neutrino Scattering Experiment on the Booster Neutrino Beamline: FINeSSE
}

\author{
March 21, 2005
}

L. Bugel, J. M. Conrad, M. H. Shaevitz, G. P. Zeller

Columbia University, Nevis Labs, Irvington, NY 10533

S. Brice, D. Finley, R. Stefanski

Fermi National Accelerator Laboratory, Batavia, IL 60510

J. C. Peng

University of Illinois at Urbana-Champaign, Urbana, IL 61801

J. Doskow, C. Horowitz, T. Katori, H. O. Meyer, M. Novak, C. Polly, R. Tayloe*, G. Visser Indiana University, Bloomington, IN 47408

C. Green, G. T. Garvey, W. C. Louis, G. McGregor, H. Ray, R. Van de Water Los Alamos National Laboratory, Los Alamos, NM 87545

W. Metcalf, M. O. Wascko

Louisiana State University, Baton Rouge, LA 70803

V. Papavassiliou, S. F. Pate

New Mexico State University, Las Cruces, NM 88003

A. Curioni, B. T. Fleming*

Yale University, New Haven, CT 06520

* Co-spokespersons: B. T. Fleming and R. Tayloe 


\title{
A Letter of Intent for a Neutrino Scattering Experiment on the Booster Neutrino Beamline: FINeSSE
}

\author{
March 21, 2005 \\ L. Bugel, J. M. Conrad, M. H. Shaevitz, G. P. Zeller \\ Columbia University, Nevis Labs, Irvington, NY 10533 \\ S. Brice, D. Finley, R. Stefanski \\ Fermi National Accelerator Laboratory, Batavia, IL 60510 \\ J. C. Peng \\ University of Illinois at Urbana-Champaign, Urbana, IL 61801 \\ J. Doskow, C. Horowitz, T. Katori, H. O. Meyer, M. Novak, \\ C. Polly, R. Tayloe*, G. Visser \\ Indiana University, Bloomington, IN 47408 \\ C. Green, G. T. Garvey, W. C. Louis, G. McGregor, H. Ray, R. Van de Water \\ Los Alamos National Laboratory, Los Alamos, NM 87545 \\ W. Metcalf, M. O. Wascko \\ Louisiana State University, Baton Rouge, LA 70803 \\ V. Papavassiliou, S. F. Pate \\ New Mexico State University, Las Cruces, NM 88003 \\ A. Curioni, B. T. Fleming* \\ Yale University, New Haven, CT 06520 \\ * Co-spokespersons: B. T. Fleming and R. Tayloe
}




\section{Contents}

1 Executive Summary 1

2 Physics Motivation $\quad 5$

$2.1 \Delta s$ Measurement . . . . . . . . . . . . . . . . . . 6

2.1.1 $\Delta s$ : Strange Matrix Element of the Axial Current . . . . . . . 7

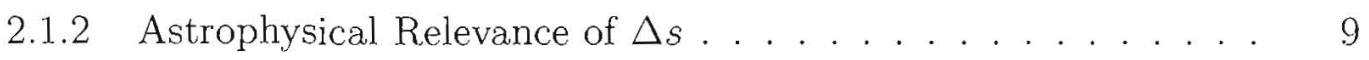

2.1.3 Current Information on $\Delta s \ldots \ldots . . \ldots 11$

2.1.4 Ongoing Experimental Programs Relevant to a Measurement of $\Delta s \ldots \ldots \ldots \ldots \ldots$

2.1.5 A FNAL Measurement of $\Delta s$ Using $\mathrm{NC}$ and $\mathrm{CC} \nu N$ and $\bar{\nu} N$ Scattering ....................... 21

2.2 Neutrino Cross Sections . . . . . . . . . . . . . . . . . 27

2.2.1 Motivation for Improved Cross Section Measurements . . . . . 28

2.2.2 Present Understanding . . . . . . . . . . . . . . 29

2.2.3 Prospects for Measuring Cross Sections: Some Examples . . . 31

2.2.4 Global Importance . . . . . . . . . . . . . . . . . 35

3 The Neutrino Beam and Expected Event Rates 37

3.1 The Booster Neutrino Beam . . . . . . . . . . . . . . . . 37

3.2 Booster Neutrino Beam Production . . . . . . . . . . . . . . 38

3.3 Event Rates . . . . . . . . . . . . . . . . . . . . 40 
4 The Detector $\quad 45$

4.1 Detector Overview ......................... 45

4.2 The Vertex Detector . . . . . . . . . . . . . . . . 46

4.2 .1 Prototype Tests . . . . . . . . . . . . . . . 50

4.2 .2 Neutron Tagging . . . . . . . . . . . . . . 61

4.3 The Muon Rangestack . . . . . . . . . . . . . . . . . . . . . . . 62

4.4 Signal Readout: Phototubes and Electronics . . . . . . . . . . . . 63

4.5 A Possible Future Upgrade: Liquid Argon Time Projection Chamber 64

4.5.1 Detection Technique and Detector Construction . . . . . . . . 65

4.5.2 R\&D program . . . . . . . . . . . . . . 66

5 Physics Sensitivity $\quad 69$

5.1 Introduction to Interactions in the Detector . . . . . . . . . . . 69

5.2 Physics Simulations . . . . . . . . . . . . . . . . . 74

5.2.1 Simulation of the Detector . . . . . . . . . . . . 74

5.2.2 Event Reconstruction . . . . . . . . . . . . . . . . 77

5.3 Physics Sensitivities . . . . . . . . . . . . . . . . . 82

5.3.1 $\Delta s$ Measurement . . . . . . . . . . . . . . . . . 82

5.3.2 Neutrino Cross Section Measurements . . . . . . . . . . . . 90

$\begin{array}{lll}6 & \text { Implementation at Fermilab } & 95\end{array}$

$\begin{array}{llr}7 & \text { Conclusions } & 97\end{array}$

$\begin{array}{lr}\text { Bibliography } & 99\end{array}$ 


\section{Chapter 1}

\section{Executive Summary}

The experiment described in this Letter of Intent provides a decisive measurement of $\Delta s$, the spin of the nucleon carried by strange quarks. This is crucial as, after more than thirty years of study, the spin contribution of strange quarks to the nucleon is still not understood.

The interpretation of $\Delta s$ measurements from inclusive Deep Inelastic Scattering (DIS) experiments using charged leptons suffers from two questionable techniques; an assumption of SU(3)-flavor symmetry, and an extrapolation into unmeasured kinematic regions, both of which provide ample room for uncertain theoretical errors in the results. The results of recent semi-inclusive DIS data from HERMES paint a somewhat different picture of the contribution of strange quarks to the nucleon spin than do the inclusive results, but since HERMES does not make use of either of the above-mentioned techniques, then the results are somewhat incomparable. What is required is a measurement directly probing the spin contribution of the strange quarks in the nucleon.

Neutrino experiments provide a theoretically clean and robust method of determining $\Delta s$ by comparing the neutral current interaction, which is isoscalar plus isovector, to the charged current interaction, which is strictly isovector. A past experiment, E734, performed at Brookhaven National Laboratory, has pioneered this effort. Building on what they have learned, we present an experiment which achieves a measurement to \pm 0.025 using neutrino scattering, and \pm 0.04 using anti-neutrino scattering, significantly better than past measurements. The combination of the neutrino and anti-neutrino data, when combined with the results of the parity-violating electron-nucleon scattering data, will produce the most significant result for $\Delta s$. 
This experiment can also measure neutrino cross sections in the energy range required for accelerator-based precision oscillation measurements. Accurate measurements of cross sections have been identified as a priority of the neutrino community, as determined through the APS Multidisciplinary Study on the Future of Neutrino Physics. From the APS report, the Neutrino Matrix makes its recommendations in context of several assumptions regarding the neutrino program, including:

"Determination of the neutrino reaction and production cross sections required for a precise understanding of neutrino oscillation physics and the neutrino astronomy of astrophysical and cosmological sources. Our broad and exacting program of neutrino physics is built upon precise knowledge of how neutrinos interact with matter."

The experiment described here will provide unique information on cross sections of $\sim 1 \mathrm{GeV}$ neutrinos, in precisely the range explored by present and future long baseline oscillation programs.

Fermi National Accelerator Laboratory is the natural place to perform this experiment. The physics goals proposed here grow the existing program and are necessary ingredients for the next generation oscillation physics measurements in this same energy range. This is a small, cost-effective, and timely experiment which fits well with the growing neutrino program at Fermilab.

\section{Outline}

The experiment is presented in the following order:

- Chapter 2 provides the physics motivation for these measurements, establishing that a new neutrino experiment is necessary;

- Chapter 3 describes the flux and event rates at this near location on the Booster Neutrino Beamline;

- Chapter 4 sets forth detection techniques, demonstrating that an appropriate detector can be built on a reasonable time scale for $\$ 2.8 \mathrm{M}$, including contingency; 
- Chapter 5 examines neutrino interactions in the detector for the physics measurements, showing that a decisive measurement can be achieved;

- Chapter 6 provides some details on implementation at Fermilab;

- Chapter 7 provides a summary and conclusion.

\section{Requests to the Directorate and PAC}

The purpose of this letter of intent is to lay out a program of physics which warrants encouragement from the PAC and Directorate to take the LoI to the proposal stage. We ask for your comments and encouragement. For this LoI and en route to taking FINeSSE to the proposal stage, we have made some assumptions regarding beam rate, funding scenarios and time-line. These are outlined below. We ask for your specific comments on these assurnptions so we may modify them as necessary in preparing a proposal and planning the trajectory of the experiment.

- We have assumed a beam delivery rate of $2 \times 10^{20}$ protons on target (POT) per year based on a letter from Mike Witherell from August, 2004. Our run plan will need to be modified should this POT rate change. We would like to know this "design" POT number as well as a "minimum" possible number of $\mathrm{POT} /$ year in order to prepare a proposal.

- Funding scenarios include pursuing outside funding for detector materials and construction, however, we will request that the lab construct the detector hall to house the experiment and help, if possible, with some of the detector costs.

- Provided funding is aquired in a timely manner, the FINeSSE run will start in 2008 and span approximately 3 years in order to accumulate $6 \times 10^{20}$ POT.

Thank you for your consideration. 


\section{Chapter 2}

\section{Physics Motivation}

This experiment will cleanly measure the strange quark contribution to the nucleon spin, $\Delta s$, as well as a suite of neutrino and anti-neutrino cross sections in the crucial $1 \mathrm{GeV}$ energy region.

The strange quark contribution to the nucleon spin remains unresolved after several decades of work via deep-inelastic scattering experiments. Neutrino neutralcurrent (NC) elastic scattering is uniquely sensitive to the nucleon axial structure and offers the best method for extracting $\Delta s$. Only one previous neutrino scattering experiment to date, BNL E734 [1], has attempted to measure $\Delta s$, albeit with limited precision and sensitivity. A next generation neutrino scattering experiment described here is crucial to cleanly determining $\Delta s$.

In addition to $\mathrm{NC}$ elastic scattering, this experiment can measure a full palette of neutrino and anti-neutrino interactions at low energy $(\approx 1 \mathrm{GeV})$. Cross section data on such reactions are sparse. Improved knowledge of low energy neutrino interaction rates on a nuclear target is becoming increasingly important as neutrino oscillation experiments in this energy range enter the precision era.

This chapter motivates these measurements, describing their history and presenting the improved precision and sensitivity this experiment can achieve. 


\section{$2.1 \Delta s$ Measurement}

From the time that the composite nature of the proton was discovered, physicists have sought to understand its constituents. The study of nucleon spin has grown into an industry, and opened new theoretical frontiers. Deep-Inelastic Scattering (DIS) measurements with polarized beams and/or targets have given us a direct measurement of the spins carried by the quarks in the nucleon. A central mystery has unfolded: in the nucleon, if the $u$ and $d$ valence quarks carry approximately equal and opposite spins, where lies the remainder?

One key contribution that has eluded a definitive explanation is the spin contribution from strange quarks in the nucleon sea. A large strange quark spin component, extracted from recent measurements [2], would be of great theoretical interest, since it would require significant changes to current assumptions. Is this large value of the strange spin due to chiral solitons [3], a misinterpretation of the large gluon contributions coming from the QCD axial anomaly [4, 5], or incorrect assumptions of SU(3) symmetry [6]? In addition, an understanding of the nucleon spin structure is a key input to dark matter searches and to the understanding of core collapse supernovae.

It has been known for some time that low energy (and low- $Q^{2}$ ) neutrino measurements are a theoretically robust technique (as robust as, e.g., the Bjorken sum rule) for isolating the strange quark contribution. Low-energy, intense neutrino beams now make it possible to take greater advantage of this method. The experiment described here, using these beams along with a novel detection technique, will resolve the presently murky experimental picture.

This experiment will measure $\Delta s$ by examining neutral current neutrino-proton scattering; the rate of this process is sensitive to any contributions from strange quarks (both $s$ and $\bar{s}$ ) to the nucleon spin. Specifically, $\Delta s$ is extracted from the ratio of neutral current neutrino-proton $(\nu p \rightarrow \nu p)$ scattering to charged current neutrino-neutron $\left(\nu n \rightarrow \mu^{-} p\right.$ ) scattering. The measurement will be performed at low momentum transfer $\left(Q^{2} \approx 0.2 \mathrm{GeV}^{2}\right)$, in order to unambiguously extract $\Delta s$ from the axial form factor, $G_{A}$. We will improve on the latest measurement of neutral current neutrino-proton scattering (BNL 734) [1] by measuring this process not only at a lower- $Q^{2}$, but also with more events, less background, and lower systematic uncertainty.

In the following sections, we describe previous and current experiments relevant 
to the question of strange quarks in the nucleon. We then describe why neutral current neutrino-nucleon elastic scattering is sensitive to the axial structure of the nucleon. We show a recent analysis of how the data may be analyzed together with PV electron scattering data. We conclude with a summary of the sensitivity of this experiment to $\Delta s$ (detailed more completely in Chapter 5 ).

\subsection{1 $\Delta s$ : Strange Matrix Element of the Axial Current}

In the current experimental program of nucleon structure studies, we find two broad areas of experimentation. First, elastic scattering of electrons from nucleons is used to measure the electroweak and axial form factors of the nucleon over a range of momentum transfer of $0.1<Q^{2}<10 \mathrm{GeV}^{2}$. These experiments have taken place at a variety of laboratories over the years, with the current program focused at MIT-Bates, JLab, and Mainz. The emphasis of the current program has been on determining the strange quark contributions to the electromagnetic form factors, through the exploitation of the interference between photon and $Z$-boson exchange processes. Second, deep-inelastic scattering of muons and electrons from nucleon and nuclear targets, historically responsible for the discovery of the partonic structure of matter, continues to play a role in the exploration of the distribution of quarks and gluons in nucleons. One of the highlights here is the focus, over the last 15 years, on the spin structure of the nucleon. The deep-inelastic exploration of nucleon spin takes place now at both leptonic and hadronic facilities - the spin program at RHIC being the most notable example of an hadronic facility taking on this physics topic.

QCD provides a simple framework in which these two experimental programs are joined together. The asymmetries observed in the polarized deep-inelastic scattering experiments arise from the antisymmetric part of the virtual Compton amplitude, which contains at its heart the nucleon axial current, $\bar{q} \gamma_{\mu} \gamma_{5} q$. In the quark-parton model, inclusive scattering of leptons from nucleon targets measures the nucleon structure function $F_{1}$,

$$
F_{1}(x)=\frac{1}{2} \sum_{q} e_{q}^{2} q(x)
$$

where $e_{q}$ and $q(x)$ are respectively the charge and parton distribution function for quarks of flavor $q$. Inclusive scattering of polarized leptons from polarized nucleon targets measures the spin-dependent nucleon structure function $g_{1}$,

$$
g_{1}(x)=\frac{1}{2} \sum_{q} e_{q}^{2} \Delta q(x)
$$


where now $\Delta q(x)$ is a polarized parton distribution function; it is defined as $\Delta q(x)=$ $q^{\dagger}(x)-q^{\downarrow}(x)$, where $q^{\dagger}(x)$ and $q^{\downarrow}(x)$ are respectively the distributions for quarks to be found parallel or anti-parallel to the proton spin. In QCD, these distribution functions take on a scale dependence: $\Delta q\left(x, Q^{2}\right)$. At the same time, the axial form factors $G_{A}^{q}\left(Q^{2}\right)$ measured in elastic neutrino scattering are themselves matrix elements of the axial current,

$$
{ }_{N}\left\langle p^{\prime}\left|\bar{q} \gamma_{\mu} \gamma_{5} q\right| p\right\rangle_{N}=\bar{u}\left(p^{\prime}\right) \gamma_{\mu} \gamma_{5} G_{A}^{q}\left(Q^{2}\right) u(p)
$$

where the matrix element has been taken between two nucleon states of momenta $p$ and $p^{\prime}$, and $Q^{2}=-\left(p^{\prime}-p\right)^{2}$. The diagonal matrix elements of the axial current are called the axial charges,

$$
{ }_{N}\left\langle p\left|\bar{q} \gamma_{\mu} \gamma_{5} q\right| p\right\rangle_{N}=2 M s_{\mu} \Delta q
$$

where $M$ and $s_{\mu}$ are respectively the mass and spin vector of the nucleon. The quantities $\Delta q$ are called "axial charges" because they are the value of the axial form factors at $Q^{2}=0$; that is to say, for example, $G_{A}^{s}\left(Q^{2}=0\right)=\Delta s$. The connection between the two sets of observables lies in a well-known QCD sum rule for the axial current, namely that the value of the axial form factor at $Q^{2}=0$ is equal to the integral over the polarized parton distribution function measured at $Q^{2}=\infty$. For example,

$$
\Delta s=G_{A}^{s}\left(Q^{2}=0\right)=\int_{0}^{1} \Delta s\left(x, Q^{2}=\infty\right) d x .
$$

In this way, the axial charges $\Delta q$ provide the link between the low-energy elastic neutrino scattering measurements of axial form factors and the high-energy deepinelastic measurements of polarized parton distribution functions.

Of course, there are practical difficulties in the full exploration of this sum rule. No scattering experiment can reach $Q^{2}=0$ or $Q^{2}=\infty$, and no deep-inelastic experiment can ever reach $x=0$. However, the consequences of these difficulties are more severe in some cases than in others. The inability to reach $Q^{2}=\infty$ in the deep-inelastic program means that QCD corrections enter into the sum rule written above. There is much theoretical experience in calculating these corrections. While the low-energy elastic experiments cannot reach $Q^{2}=0$, divergent behavior of the form factors near $Q^{2}=0$ is not expected and so the idea of extrapolating to $Q^{2}=0$ from measurements at low, non-zero $Q^{2}$ is not met with alarm. On the other hand, the limitations imposed by the inability to reach $x=0$ in the deep-inelastic experiments are more problematic. The unpolarized parton distribution functions $q(x)$ 
are all known to increase rapidly as $x \rightarrow 0$ and there is no calculation of the expected behavior near $x=0$ to rely upon for an extrapolation from measurements made at $x \neq 0$. Similar comments apply to the polarized parton distributions $\Delta q(x)$. Unpolarized measurements of the parton distributions at HERA have reached very low values of $x$, nearing $x=3 \times 10^{-5}$, but the corresponding measurements of the polarized distribution functions, from data at SLAC, CERN, and DESY, only reach $x=3 \times 10^{-3}$. Therefore, measurements of the axial charges place important constraints on the behavior of the distributions $\Delta q(x)$ in the unmeasurable low- $x$ region.

As this Letter of Intent will demonstrate, the only way to measure the strange axial charge, $\Delta s$, is by means of low-energy elastic scattering of neutrinos from nucleons.

\subsubsection{Astrophysical Relevance of $\Delta s$}

$\Delta s$ is a fundamental property of the nucleon that is not well-known and will lead to much insight into nucleon structure. In addition, it is a quantity that needs to be better measured to understand two important astrophysical questions.

\section{Core Collapse Supernovae}

Core collapse supernovae (SN) are massive explosions of single stars that are dominated by neutrinos and their weak interactions. Ninety-nine percent of the total energy released is radiated in neutrinos. The mean free path for $\mu$ and $\tau$ neutrinos is dominated by neutrino-nucleon elastic scattering. These neutrinos do not have enough energy for charged current interactions that require producing a charged lepton. The mean free path determines the diffusion time and indeed neutrinos were observed for about 10 seconds from SN1987A. The mean free path also determines the rate of energy transfer from the hot protoneutron star to matter near the shock front. This shock is thought to be energized by neutrino interactions and eventually ejects $90 \%$ of the star to form the supernova. Such calculations are very sensitive to the details of how neutrinos interact with matter. This is because there is so much more energy in the neutrino radiation and a small change in the energy deposition can determine if the shock succeeds in exploding the star or fails and the system collapses to a black hole. A number of theorists are presently performing large scale numerical simulations of SN, see, for example, Ref. [7]. 
Much of the matter behind the shock has been dissociated into nearly free nucleons that are mostly neutrons because of rapid electron capture. Therefore, one of the most important reactions is neutrino-neutron elastic scattering. In a SN, typical neutrino energies are near $25 \mathrm{MeV}$ so momentum transfers are small. This cross section is still uncertain at the approximately $20 \%$ level because of the unknown value of the strange quark axial charge, $\Delta s$. The effect of strange quark uncertainties on neutrino opacities is discussed in Ref. [8]. The measurement described in this Letter will provide better input for such SN simulations. A smaller cross section would imply a smaller diffusion time and, other things being equal, a more rapid transport of energy from the protoneutron star to the region near the shock front.

\section{Dark Matter Searches}

Understanding the spin contribution to the nucleon of the strange quarks is important for certain searches of dark matter [9]. In $R$-parity-conserving supersymmetric models, the lightest supersymmetric particle (LSP) is stable and therefore a darkmatter candidate; in certain scenarios, the relic LSP density is large enough to be of cosmological interest. Experimental searches for cosmic LSPs can be competitive with accelerator-based searches [10].

In the case where the LSP is the neutralino, cosmic LSP can be detected either directly, through elastic neutralino scattering in an appropriate target/detector, or indirectly. The indirect method involves detecting high-energy neutrinos from neutralino annihilations in the center of the sun: neutralinos in the galactic halo can lose energy through elastic scattering with nuclei as they pass through the sun and become gravitationally trapped, sinking to the center. There, they accumulate and can subsequently annihilate into gauge bosons or heavy quarks; a significant fraction of the decay products of those involves high-energy neutrinos. The accumulation rate, and therefore the observed neutrino flux on earth, depends on the neutralino-nucleon scattering cross section (see [11] for a detailed calculation).

The neutralino-nucleus elastic-scattering cross section contains a spin-dependent and a spin-independent part. The spin-dependent part is given by

$$
\sigma=\frac{32}{\pi} G_{F}^{2} m_{r}^{2} \Lambda^{2} J(J+1)
$$

where $G_{F}$ is the Fermi constant, $m_{r}$ the reduced neutralino mass, $J$ the nucleus spin, 
and

$$
\Lambda \equiv \frac{1}{J}\left(a_{p}\left\langle S_{p}\right\rangle+a_{n}\left\langle S_{n}\right\rangle\right)
$$

here $\left\langle S_{p(n)}\right\rangle$ is the average proton (neutron) spin in the nucleus and

$$
a_{p(n)}=\sum_{i} \frac{\alpha_{i}}{\sqrt{2} G_{F}} \Delta q_{i}^{p(n)}
$$

where the sum is over quark flavors and the coefficients $\alpha_{i}$ are functions of the composition of the neutralino in terms of the supersymmetric partners of the gauge bosons. The factors $\Delta q_{i}^{p}$ and $\Delta q_{i}^{n}$ are the quark contributions to the proton or neutron spin.

It is established $[2,12]$ that $\Delta u$ and $\Delta d$ have opposite signs. Therefore from the above, it should be clear that knowledge of $\Delta s$ is not only important for the interpretation of any limits from such dark matter searches, but could also influence the choice of detector material for direct searches [13], making nuclei with either proton- or neutron-spin excess optimal, depending on its value and sign.

\subsubsection{Current Information on $\Delta s$}

The importance of measuring $\Delta s$ has been known for quite some time, yet it is not an exaggeration to say this quantity has never been measured. All information to date on $\Delta s$ is laden with questionable theoretical assumptions coupled to extrapolations of experimental data into unmeasured regions. In this section we show why a robust measurement of $\Delta s$ is long overdue.

\section{$\Delta s$ from E734}

The only extensive neutrino-nucleon elastic scattering data available are from the BNL E734 experiment [1]. This experimental program had many goals, one of which was the extraction of $\Delta s$. The experiment measured the cross sections for $\nu p \rightarrow$ $\nu p$ and $\bar{\nu} p \rightarrow \bar{\nu} p$ elastic NC scattering in seven $Q^{2}$ bins over the kinematic range $0.45<Q^{2}<1.05 \mathrm{GeV}^{2}$. The cross sections were normalized [14] to the event rate for $\nu n \rightarrow \mu^{-} p$ and $\bar{\nu} p \rightarrow \mu^{+} n$ quasi-elastic CC scattering, for which the cross sections were already known [15]. The error on these measured cross sections in the lowest $Q^{2}$ bin was $20 \%(22 \%)$ for the $\nu p \rightarrow \nu p(\bar{\nu} p \rightarrow \bar{\nu} p)$ reaction. 
Elastic neutrino-nucleon scattering is very sensitive to the axial form factor of the nucleon. The cross section for $\nu p$ and $\bar{\nu} p$ elastic scattering is given by [16]

$$
\frac{d \sigma}{d Q^{2}}=\frac{G_{F}^{2}}{2 \pi} \frac{Q^{2}}{E_{\nu}^{2}}\left(A \pm B W+C W^{2}\right)
$$

where the $+(-)$ sign is for $\nu(\bar{\nu})$ scattering, and

$$
\begin{aligned}
W & =4\left(E_{\nu} / M_{p}-\tau\right) \\
\tau & =Q^{2} / 4 M_{p}^{2} \\
A & =\frac{1}{4}\left[\left(G_{A}^{Z}\right)^{2}(1+\tau)-\left(\left(F_{1}^{Z}\right)^{2}-\tau\left(F_{2}^{Z}\right)^{2}\right)(1-\tau)+4 \tau F_{1}^{Z} F_{2}^{Z}\right] \\
B & =-\frac{1}{4} G_{A}^{Z}\left(F_{1}^{Z}+F_{2}^{Z}\right) \\
C & =\frac{1}{64 \tau}\left[\left(G_{A}^{Z}\right)^{2}+\left(F_{1}^{Z}\right)^{2}+\tau\left(F_{2}^{Z}\right)^{2}\right] .
\end{aligned}
$$

Here, $E_{\nu}$ is the neutrino beam energy, and $F_{1}^{Z}, F_{2}^{Z}$, and $G_{A}^{Z}$ are respectively the neutral weak Dirac, Pauli, and axial form factors. At low $Q^{2}$, this cross section is dominated by the axial contribution:

$$
\frac{d \sigma}{d Q^{2}}\left(Q^{2}=0\right)=\frac{G_{F}^{2}}{128 \pi} \frac{M_{p}^{2}}{E_{\nu}^{2}}\left[(-\Delta u+\Delta d+\Delta s)^{2}+\left(1-4 \sin ^{2} \theta_{W}\right)^{2}\right] .
$$

The quantity $\Delta u-\Delta d$ is already known from neutron $\beta$ decay; this is just $g_{A}$, the $Q^{2}=0$ value of the CC part of the axial form factor. So, the only unknown part is the strange axial charge, $\Delta s$. Of course, the measurement cannot be performed at $Q^{2}=0$ so a determination of $\Delta s$ will always involve an extrapolation over some unmeasured interval near $Q^{2}=0$.

In their $\Delta s$ analysis the E734 collaboration use a dipole form for the weak axial form factor,

$$
G_{A}^{Z}\left(Q^{2}\right)=\frac{1}{2} \frac{g_{A}(1+\eta)}{\left(1+\overline{\left.Q^{2} / M_{A}^{2}\right)^{2}}\right.}
$$

where $g_{A}=1.26$ is the $\mathrm{CC}$ coupling constant measured in neutron $\beta$ decay, $M_{A}$ is the "axial mass" parameter used in fitting the $Q^{2}$-dependence of the known (CC) part of the axial form factor, and $\eta$ is a parameter used to measure the size of strange quark contributions to the axial form factor. In our modern notation, $\Delta s=-\eta g_{A}$. It is important to note that while the dipole shape fits the $\mathrm{CC}$ part of the axial form factor nicely, this form has no physical significance and there is no theoretical support for the idea that the strange sea contribution to the form factor should have 


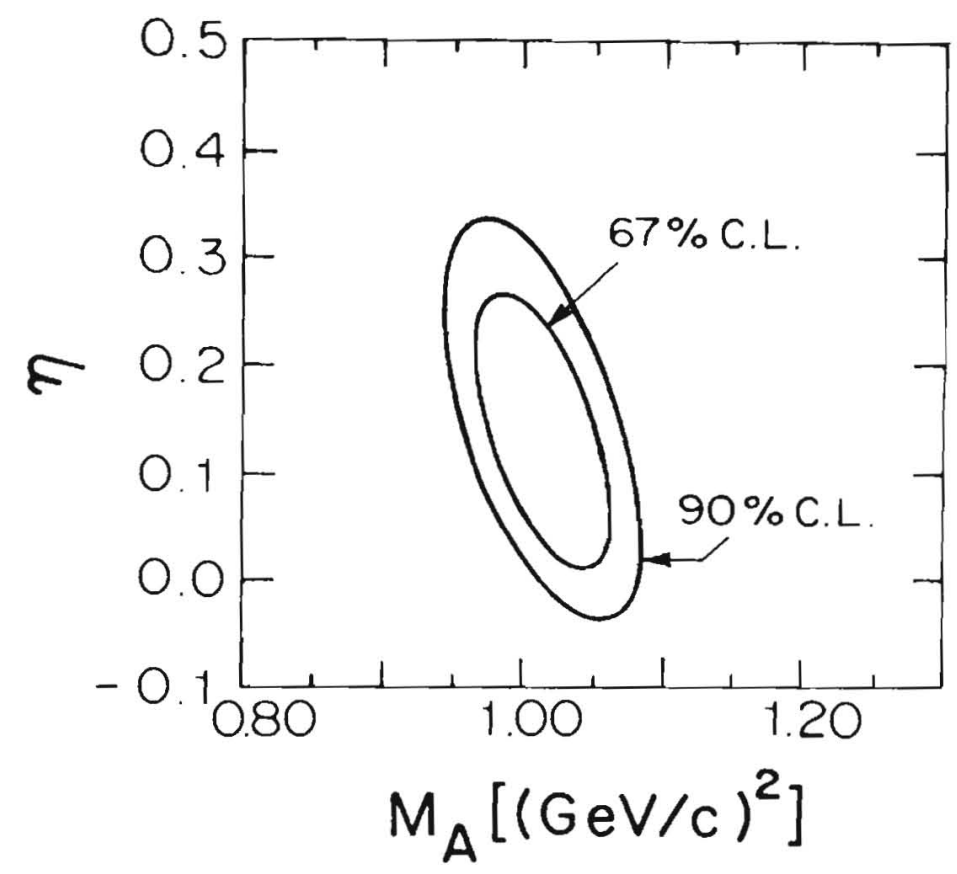

Figure 2.1: Results from a fit of the BNL E734 $\nu p \rightarrow \nu p$ and $\bar{\nu} p \rightarrow \bar{\nu} p$ scattering data [1] indicating the preferred values of $\eta\left(=-\Delta s / g_{A}\right)$ and $M_{A}$. (Figure from Ref. [1].)

the same $Q^{2}$-dependence as the up- and down-quark valence contribution. Fitting their data to extract $\eta$, with $M_{A}$ at the world average at that time, they found a value $\eta=0.12 \pm 0.07$, implying a value of $\Delta s=-0.15 \pm 0.09$. A simultaneous fit to both $\eta$ and $M_{A}$ yields a value of $\eta$ with a substantially larger error as may be seen from the fit results, shown in Figure 2.1. The current world average neutrino scattering data give $M_{A}=1.001 \pm 0.020 \mathrm{GeV}[17,18]$.

Many years later, Garvey, Louis and White [16] performed a reanalysis of the E734 data introducing a number of additional features, compared to the original E734 analysis:

- Allowed for non-zero values of the vector strange form factors, which had been set to zero in the E734 analysis.

- Introduced a floating normalization for each data point, to allow for the uncertainty in the nuclear corrections made in the E734 cross section extraction procedure. 
- Included more recent nucleon form factor values, while continuing to assume the strange and non-strange axial form factors shared the same dipole form with the same parameter $M_{A}$.

Even with these improvements, this analysis resulted in a measurement of $\Delta s$ with large errors due the limited precision of the E734 data and the large uncertainty arising from the extrapolation to $Q^{2}=0$. The results from a simultaneous fit of $\Delta s$, the two strange vector form factors, $\left(F_{1}^{s}\right.$ and $\left.F_{2}^{s}\right)$, and constraining $M_{A}$ to the world-average at that time, yielded $\Delta s=-0.21 \pm 0.10$. However, satisfactory fits were obtained for $\Delta s$ ranging from 0 to -0.21 depending on the details of the $Q^{2}$ evolution of the axial form factor.

More recently, Alberico et al. [19] performed a rather different analysis using the E734 data, again with the goal of extracting the strange form factors of the proton. Instead of a point-to-point analysis and fit of the data, they focused their attention upon the use of several integral ratios reported by E734, namely

$$
\begin{aligned}
& R_{\nu}=\frac{\langle\sigma\rangle_{\nu p \rightarrow \nu p}}{\langle\sigma\rangle_{\nu n \rightarrow \mu^{-} p}}=0.152 \pm 0.007 \text { (stat) } \pm 0.017 \text { (syst) } \\
& R_{\bar{\nu}}=\frac{\langle\sigma\rangle_{\bar{\nu} p \rightarrow \bar{\nu} p}}{\langle\sigma\rangle_{\bar{\nu} p \rightarrow \mu^{+} n}}=0.218 \pm 0.012 \text { (stat) } \pm 0.023 \text { (syst) } \\
& R_{\nu / \bar{\nu}}=\frac{\langle\sigma\rangle_{\nu p \rightarrow \nu p}}{\langle\sigma\rangle_{\bar{\nu} p \rightarrow \bar{\nu} p}}=0.302 \pm 0.019 \text { (stat) } \pm 0.037 \text { (syst) }
\end{aligned}
$$

where each $\langle\sigma\rangle$ is an average over the range $0.5<Q^{2}<1.0 \mathrm{GeV}^{2}$. These three ratios are then combined into a single ratio,

$$
\left\langle A_{p}\right\rangle=\frac{\langle\sigma\rangle_{\nu p \rightarrow \nu p}-\langle\sigma\rangle_{\bar{\nu} p \rightarrow \bar{\nu} p}}{\langle\sigma\rangle_{\nu n \rightarrow \mu^{-} p}-\langle\sigma\rangle_{\bar{\nu} p \rightarrow \mu^{+} n}}=\frac{R_{\nu}\left(1-R_{\nu / \bar{\nu}}\right)}{1-R_{\nu / \bar{\nu}} R_{\nu} / \bar{R}_{\bar{\nu}}} .
$$

This ratio method allows for cancellation of any multiplicative errors associated with nuclear effects in the extraction of the original cross sections in E734. While this analysis pointed out the power of ratios in reducing nuclear model uncertainties, the errors on the E734 data did not allow a precise extraction of $\Delta s$ and the conclusions were consistent with the Garvey, Louis, White reanalysis of the data described above [16], and led to no additional insight on the value of $\Delta s$.

As is evident in this discussion, the BNL E734 data has generated a large amount of interest; this points to growing appreciation of the fact that neutral current neutrino scattering is an excellent probe of $\Delta s$. Unfortunately, however, the BNL E734 data are not precise enough to determine $\Delta s$. 


\section{$\Delta s$ from Charged-Lepton Deep-inelastic Scattering}

As mentioned earlier, the double-spin asymmetries in polarized inclusive leptonic deep-inelastic scattering measure the spin-dependent nucleon structure function $g_{1}$ :

$$
g_{1}(x)=\frac{1}{2} \sum_{q} e_{q}^{2} \Delta q(x)
$$

In leading order QCD, these functions take on a scale dependence:

$$
g_{1}\left(x, Q^{2}\right)=\frac{1}{2} \sum_{q} e_{q}^{2} \Delta q\left(x, Q^{2}\right) .
$$

In next-to-leading order (NLO) QCD, there are significant radiative corrections and the relation between $g_{1}$ and the $\Delta q$ becomes more complex. In the discussion here, we limit our attention to the leading-order QCD analysis because the NLO version of the analysis does not change the result (nor the uncertainty) for $\Delta s$ very much, and the problems to be pointed out exist at all orders, because they are problems stemming from the data itself.

Consider the analysis from the SMC Collaboration [12] as an example. They measured $g_{1}\left(x, Q^{2}\right)$ over a wide kinematic range, $0.003<x<0.70$ and $1.3<Q^{2}<$ $58.0 \mathrm{GeV}^{2}$. This coverage is not a rectangle, i.e. there is a correlation between $x$ and $Q^{2}$ in the acceptance of the experiment, and so for a reasonable analysis it is necessary to use QCD to evolve all the data to a single value of $Q^{2}$, in this case $Q^{2}=10 \mathrm{GeV}^{2}$. In the process of performing this evolution, a fit function for $g_{1}$ is produced. Then, to integrate the distribution $g_{1}$ over $0<x<1$, it is necessary to extrapolate to $x=1$ and $x=0$. The extrapolation to $x=1$ makes use of the fact that $g_{1}$, being a difference of two quark distributions, must go to 0 as $x \rightarrow 1$. This requirement is satisfied by assuming the measured experimental asymmetry to be constant for $x>0.7$. The extrapolation to $x=0$, on the other hand, is not straightforward, as the expected behavior of $g_{1}(x)$ for $x \rightarrow 0$ is unknown. In this analysis, two methods were used. In one, the $\mathrm{QCD}$ evolution fit was simply extrapolated to $x=0$. In another, called the "Regge extrapolation," the value of $g_{1}$ was assumed to be constant for $x<0.003$. The results of these extrapolations may be seen in Fig. 2.2. The two values of the integral of $g_{1}$ from these extrapolations are

$$
\begin{aligned}
\Gamma_{1}=\int_{0}^{1} g_{1}(x) d x & =0.142 \pm 0.017 \quad \text { "Regge" } \\
& =0.130 \pm 0.017 \quad \text { QCD fit. }
\end{aligned}
$$




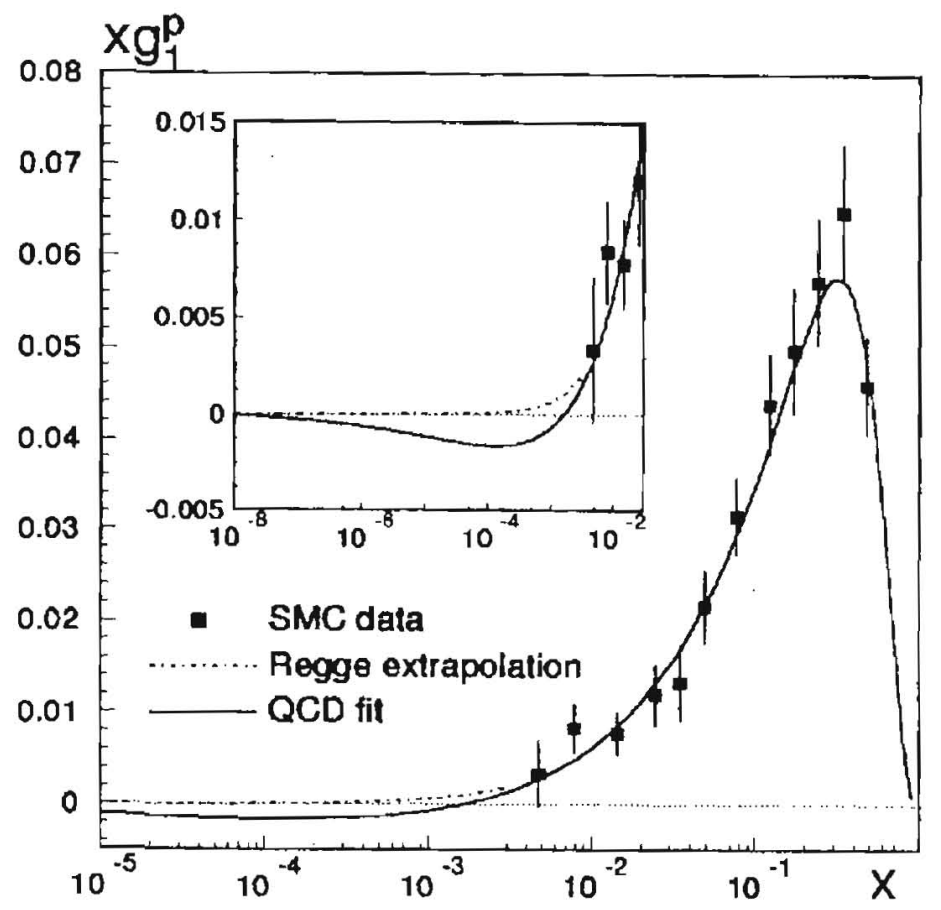

Figure 2.2: Results of the SMC measurements of $g_{1}\left(x, Q^{2}\right)$. Plotted is $x g_{1}$ as a function of $x$. The QCD fit is the continuous line, while the extrapolation assuming Regge behavior is indicated by the dot-dashed line. The inset is a close-up extending to lower values of $x$. (Figure from Ref. [12].)

This integral is related to the axial charges:

$$
\Gamma_{1}=\int_{0}^{1} g_{1}(x) d x=\frac{1}{2} \sum_{q} e_{q}^{2} \int_{0}^{1} \Delta q(x) d x=\frac{1}{2}\left[\frac{4}{9} \Delta u+\frac{1}{9} \Delta d+\frac{1}{9} \Delta s\right] .
$$

Now, assuming that $\mathrm{SU}(3)_{f}$ is a valid symmetry of the baryon octet, and using hyperon $\beta$ decay data, then two other relations between the three axial charges are determined:

$$
\Delta u-\Delta d=g_{A}=F+D \quad \text { and } \quad \Delta u+\Delta d-2 \Delta s=3 F-D
$$

where $g_{A}=1.2601 \pm 0.0025$ and $F / D=0.575 \pm 0.016$ (in 1997). Now one may solve for the axial charges, yielding the results shown in Table 2.1. Of course, the error bars quoted here do not include any estimate of the theoretical uncertainty underlying the assumption of $\mathrm{SU}(3)_{f}$ syrnmetry. They do include an estimate of the uncertainty 
Table 2.1: Results for the axial charges from the SMC analysis [12] of their inclusive DIS data.

\begin{tabular}{l|r|r}
\hline \hline & \multicolumn{1}{|c|}{ "Regge" } & \multicolumn{1}{c}{ QCD fit } \\
\hline$\Delta u$ & $0.84 \pm 0.06$ & $0.80 \pm 0.06$ \\
$\Delta d$ & $-0.42 \pm 0.06$ & $-0.46 \pm 0.06$ \\
$\Delta s$ & $-0.08 \pm 0.06$ & $-0.12 \pm 0.06$ \\
\hline \hline
\end{tabular}

due to the extrapolations, but of course that is only an estimate because the actual behavior of $g_{1}$ is unknown in the $x \rightarrow 0$ region. The only conclusion to be drawn for $\Delta s$ from this analysis is that it may be negative, with a value anywhere in the range from 0 to -0.18 .

\section{$\Delta s(x)$ from Semi-inclusive Leptonic Deep-inelastic Scattering}

In semi-inclusive deep-inelastic scattering, a leading hadron is observed in coincidence with the scattered lepton. This allows a statistical identification of the struck quark, and hence a measurement of the $x$-dependence of the individual $\Delta q(x)$ distributions. (Inclusive scattering only measures the total structure function $g_{1}(x)$.) The HERMES Experiment [20] on the HERA ring at DESY was especially designed to make this measurement. HERMES measured double-spin asymmetries in the production of charged hadrons in polarized deep-inelastic scattering of positrons from polarized targets; specifically, the asymmetry in the production of charged pions on targets of hydrogen and deuterium, and of charged kaons in scattering from deuterium. There is no assumption of $\mathrm{SU}(3)_{f}$ symmetry in their analysis. They extract the following quark polarization distributions, over the range $0.023<x<0.60$ [21]:

$$
\frac{\Delta u}{u}(x) \quad \frac{\Delta d}{d}(x) \quad \frac{\Delta \bar{u}}{\bar{u}}(x) \quad \frac{\Delta \bar{d}}{\bar{d}}(x) \quad \frac{\Delta s}{s}(x)
$$

where $\frac{\Delta s}{s}(x)$ is defined to be the sum of $\frac{\Delta s}{s}(x)$ and $\frac{\Delta \bar{s}}{\bar{s}}(x)$. The results are shown in Fig. 2.3.

It is seen that within the measured uncertainties, and within the measured $x$ region, the valence quarks ( $u$ and $d)$ are polarized and the sea quarks $(\bar{u}, \bar{d}$, and $s)$ are unpolarized. The integral value of the measured polarized strange quark distribution 


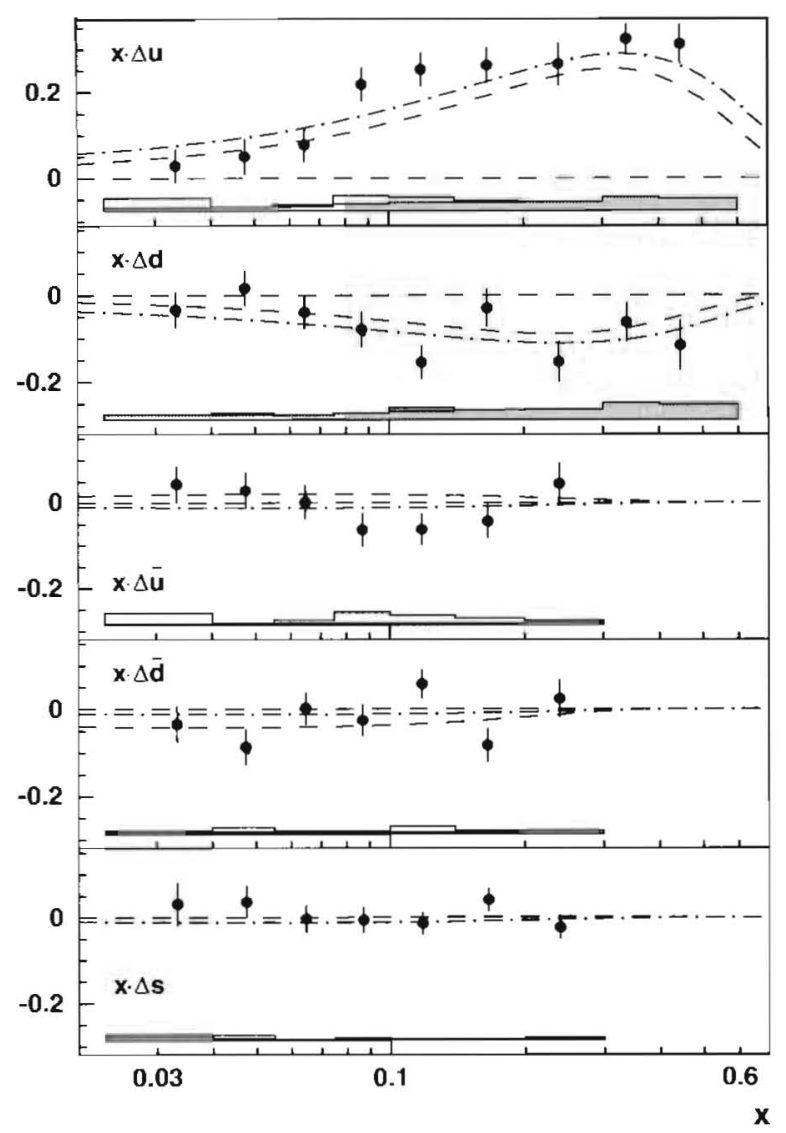

Figure 2.3: HERMES results for polarized parton distribution functions. (Figure from Ref. [21.].)

is

$$
" \Delta s "=\int_{x=0.023}^{0.60} \Delta s(x) d x=+0.028 \pm 0.033 \text { (stat) } \pm 0.009 \text { (syst) } .
$$

Note this would only be the true $\Delta s$ if the integral was over the full range, $0<x<1$.

Given the fact that the inclusive analysis described in the previous section produced a negative value of $\Delta s$, it is natural to ask "where did the negative $\Delta s$ go?" If the analyses of the inclusive and semi-inclusive data are both correct, then all the negative contribution to the value of $\Delta s$ must come from the unmeasured $x$-region, that is from $x<0.023$. That would imply an average value of $\Delta s(x)$ of approximately -5 in the range $x<0.023$, which is not impossible, as $s(x)$ is of order 20-300 in the range $0.001<x<0.01$ [22]. Some very interesting physics indeed would be revealed, if the "turn on" of the strange quark polarization in the low- $x$ region was this dramatic. 
Of course, there are other explanations. The invocation of $\mathrm{SU}(3)_{f}$ symmetry in the analysis of the inclusive data is known to be problematic and the extrapolations to $x=0$ in those analyses do not have firm theoretical support. It is clear that a direct measurement of $\Delta s$ would serve to clarify these issues.

\subsubsection{Ongoing Experimental Programs Relevant to a Mea- surement of $\Delta s$}

\section{Parity-violating $\vec{e} N$ Elastic Scattering}

One of the highlights of the current low- and medium-energy electron scattering program is the measurement of the strange vector form factors of the nucleon via parity-violating $\vec{e} N$ scattering. These measurements are sensitive as well to the nonstrange part of the axial form factor, but rather insensitive to the strange axial form factor due to the relative sizes of kinematic factors multiplying the various form factors that contribute to the asymmetry. To be specific, the parity-violating asymmetry observed in these experiments, when the target is a proton, can be expressed as [23]

$$
A_{p}=\left[\frac{-G_{F} Q^{2}}{4 \pi \alpha \sqrt{2}}\right] \frac{\epsilon G_{E}^{\gamma} G_{E}^{Z}+\tau G_{M}^{\gamma} G_{M}^{Z}-\left(1-4 \sin ^{2} \theta_{W}\right)}{\epsilon\left(G_{E}^{\gamma}\right)^{2}+\tau\left(G_{M}^{\gamma}\right)^{2}} \underline{G_{A}^{e}}
$$

where $G_{E(M)}^{\gamma}$ are the traditional electric (magnetic) form factors of the proton and $G_{E(M)}^{Z}$ are their weak (Z-exchange) analogs, $\tau=Q^{2} / 4 M_{p}^{2}, M_{p}$ is the mass of the proton, $\epsilon=\left[1+2(1+\tau) \tan ^{2}(\theta / 2)\right]^{-1}, \theta$ is the electron scattering angle, and $\epsilon^{\prime}=$ $\sqrt{\tau(1+\tau)\left(1-\epsilon^{2}\right)}$. Lastly, $G_{A}^{e}$ is the effective axial form factor seen in electron scattering:

$$
G_{A}^{e}=-G_{A}^{C C}\left(1+R_{A}^{T=1}\right)+G_{A}^{S}+R_{A}^{T=0}
$$

Here, $G_{A}^{C C}$ is the non-strange (CC) axial form factor, $G_{A}^{s}$ is the strange axial form factor, and the terms $R_{A}^{T=0,1}$ represent electroweak radiative corrections $[23,24,25$, 26]. The presence of these radiative corrections clouds the interpretation of the axial term extracted from these experiments. To solve this problem, the SAMPLE [27] experiment also measured the same asymmetry on a deuterium target, in which case the relative kinematic factors of the non-strange $(T=1)$ and strange $(T=0)$ parts of the axial form factor are changed, allowing a separation of the two. However, one may show that this does not help in identifying the value of $G_{A}^{s}$, because the relative 
size of the kinematic factors for $G_{M}^{s}$ and $G_{A}^{s}$ remain the same for either target:

$$
\frac{\partial G_{M}^{s}}{\partial G_{A}^{s}}=-\left(1-4 \sin ^{2} \theta_{W}\right) \frac{\epsilon^{\prime}}{\tau} \approx-\frac{1}{2} \text { for SAMPLE. }
$$

Therefore, parity-violating $\vec{e} N$ scattering experiments can only establish a relationship between the strange magnetic and axial form factors, they cannot measure them separately.

\section{Polarized Deep-Inelastic Scattering}

The only two deep-inelastic experiments currently running or planned that will have anything to say about $\Delta s(x)$ are HERMES and the new COMPASS Experiment at CERN.

At HERMES, it would be possible to separately measure $\Delta s(x)$ and $\Delta \bar{s}(x)$ (instead of the lumped $s$ and $\bar{s}$ distribution in the present set of results) if the asymmetry in the production of kaons from hydrogen (and not just deuterium) is also measured. However, it does not seem that this measurement will be performed soon (if ever).

The COMPASS Experiment [28], while for the most part focused on a measurement of the gluon polarization, is also equipped to measure the distributions $\Delta s(x)$ and $\Delta \bar{s}(x)$ down to $x=5 \times 10^{-3}$ with the same size of uncertainties as HERMES was able to achieve down to $x=0.023$ (see Fig. 2.3). This will be a very interesting measurement, but of course it will not be able to establish the value of the axial charge, for the usual reason that the extrapolation to $x=0$ contains too many uncertainties. As stated earlier, these sorts of deep-inelastic measurements are complementary to the strange axial charge measurement proposed in this Letter.

\section{Combining $\nu N$ and Parity-Violating $\vec{e} N$ Elastic Data}

A new analysis of the BNL734 data by Pate [29] combines low energy $\nu N$ data and parity violating $\vec{e} N$ elastic scattering data from HAPPEX [30]. Using the known values for the electric, magnetic, and non-strange (CC) axial form factors of the proton and neutron, the difference of the $\nu p$ and $\bar{\nu} p$ elastic cross sections is shown to be a function only of the strange magnetic and axial form factors, $G_{M}^{s}$ and $G_{A}^{s}$. At the same time, the sum of the $\nu p$ and $\bar{\nu} p$ elastic cross sections is shown to be a function

only of the strange electric and magnetic form factors, $G_{E}^{s}$ and $G_{M}^{s}$. Measurements of 
forward-angle parity-violating $\vec{e} p$ elastic scattering are shown to be largely functions only of $G_{E}^{s}$ and $G_{M}^{s}$ as well. This re-analysis uses these three combinations of data to determine all three strange form factors. This results in two possible solutions at $Q^{2}=0.5 \mathrm{GeV}^{2}$ summarized in Table 2.2 where Solution 1 is favored [29]. At the present time, there is only sufficient data at $Q^{2}=0.5 \mathrm{GeV}^{2}$ to make such a determination.

Data from the $G^{0}$ Experiment [31], recently collected and in the process of analysis, will provide additional measurements of $G_{E}^{s}$ and $G_{M}^{s}$ in the range $0.5<Q^{2}<$ 1.0 $\mathrm{GeV}^{2}$ and will allow for the extraction of $G_{A}^{s}$ from the E734 data. However, knowledge of the strange axial form factor over the range $0.5<Q^{2}<1.0 \mathrm{GeV}^{2}$ will not be sufficient for the extrapolation to $Q^{2}=0$ needed for a determination of $\Delta s$. New and more precise neutrino data at lower $Q^{2}$ are crucial for a definitive measurement.

Table 2.2: Two solutions for the strange form factors at $Q^{2}=0.5 \mathrm{GeV}^{2}$ produced from the E734 and HAPPEX data. (Table from Ref. [29].)

\begin{tabular}{c|r|r} 
& \multicolumn{1}{|c|}{ Solution 1 } & \multicolumn{1}{|c}{ Solution 2 } \\
\hline$G_{E}^{s}$ & $0.02 \pm 0.09$ & $0.37 \pm 0.04$ \\
$G_{M}^{s}$ & $0.00 \pm 0.21$ & $-0.87 \pm 0.11$ \\
$G_{A}^{s}$ & $-0.09 \pm 0.05$ & $0.28 \pm 0.10$
\end{tabular}

\subsubsection{A FNAL Measurement of $\Delta s$ Using NC and CC $\nu N$ and $\bar{\nu} N$ Scattering}

To address this need for a quality measurement of $\Delta s$, this Letter proposes to measure the NC to CC neutrino scattering ratio, $R_{N C / C C}=\frac{\sigma(\nu p \rightarrow \nu p)}{\sigma\left(\nu n \rightarrow \mu^{-} p\right)}$, and from it extract the strange axial form factor down to $Q^{2}=0.2 \mathrm{GeV}^{2}$. The numerator in this ratio is sensitive to the full axial form factor, $-G_{A}^{C C}+G_{A}^{s}$, while the denominator is sensitive to only $G_{A}^{C C}$. While the numerator maintains all the dependence on the strange axial form factor, measurement of the ratio reduces systematic uncertainties in neutrino flux, detector efficiency, nuclear target effects, and form factors.

In addition, it is possible to extract $\Delta s$ from a measurement of the $\mathrm{NC}$ to $\mathrm{CC}$ ratio for anti-neutrinos, $\bar{R}_{N C / C C}=\frac{\sigma(\bar{\nu} p \rightarrow \bar{\nu} p)}{\sigma\left(\bar{\nu} p \rightarrow \mu^{+} n\right)}$. This measurement is more challenging 
experimentally, since both the anti-neutrino flux and reaction rates are smaller and there is a substantial fraction of neutrinos in the anti-neutrino beam. However, the anti-neutrino ratio, $\vec{R}_{N C / C C}$, is more sensitive to $G_{A}^{s}$ and has a different $Q^{2}$ dependence as compared to the neutrino ratio, $R_{N C / C C}$. For these reasons, we are proposing to measure both of these quantities, by taking beam in both neutrino and anti-neutrino mode.

To estimate the sensitivity of the proposed experiment to $\Delta s$, a simulation of the generation and analysis of data has been performed (explained in full detail in Chapter 5). In that simulation, it is necessary to assume a $Q^{2}$-dependence for the strange axial form factor; we have assumed a dipole dependence, using the same "axial mass" parameter $M_{A}$ as is seen in the non-strange axial form factor:

$$
G_{A}^{s}=\frac{\Delta s}{\left(1+Q^{2} / M_{A}^{2}\right)^{2}} \text {. }
$$

Since the experiment and analysis will measure $G_{A}^{s}\left(Q^{2}\right)$ at several $Q^{2}$ points, we will observe the actual $Q^{2}$-dependence of $G_{A}^{s}$ with the data we collect, and will modify (if necessary) our assumption about the $Q^{2}$-dependence to complete our extraction of $\Delta s$.

The main idea of the neutrino and anti-neutrino $\mathrm{NC}$ to $\mathrm{CC}$ ratio measurements can be seen in Figures 2.4-2.5. The sensitivity of the NC elastic cross section to $\Delta s$ is shown in Figure 2.4 as a function of $Q^{2}$ for both neutrinos and anti-neutrinos. Note how the NC differential cross sections depend strongly on $\Delta s$. This strong dependence of both $R_{N C / C C}$ and $\bar{R}_{N C / C C}$ on $\Delta s$ is shown explicitly in Figure 2.5 for three $Q^{2}$ bins.

The sensitivity of the NC to CC ratio for both neutrino and anti-neutrino scattering can be quantified in the following manner. As can be seen in Fig. 2.5, the dependence of the $\mathrm{NC}$ to $\mathrm{CC}$ ratio as a function of $\Delta s$ (for small values of $\Delta s$ ) may be written as,

$$
R(\Delta s) \approx a \Delta s+b=b\left(\frac{a}{b} \Delta s+1\right)=b(\mathcal{S} \Delta s+1)
$$

where $\mathcal{S} \equiv \frac{a}{b}$, and $a$ and $b$ are the slope and intercept, respectively, of the function that describes the dependence of the NC to $\mathrm{CC}$ ratio, $R$, for neutrinos or anti-neutrinos. The (absolute) error on $\Delta s$ given an error on the (relative) error on the ratio, $R$, is then given in terms of the "sensitivity", $\mathcal{S}$,

$$
\sigma(\Delta s)=\frac{1}{|\mathcal{S}|} \frac{\sigma(R)}{R}
$$




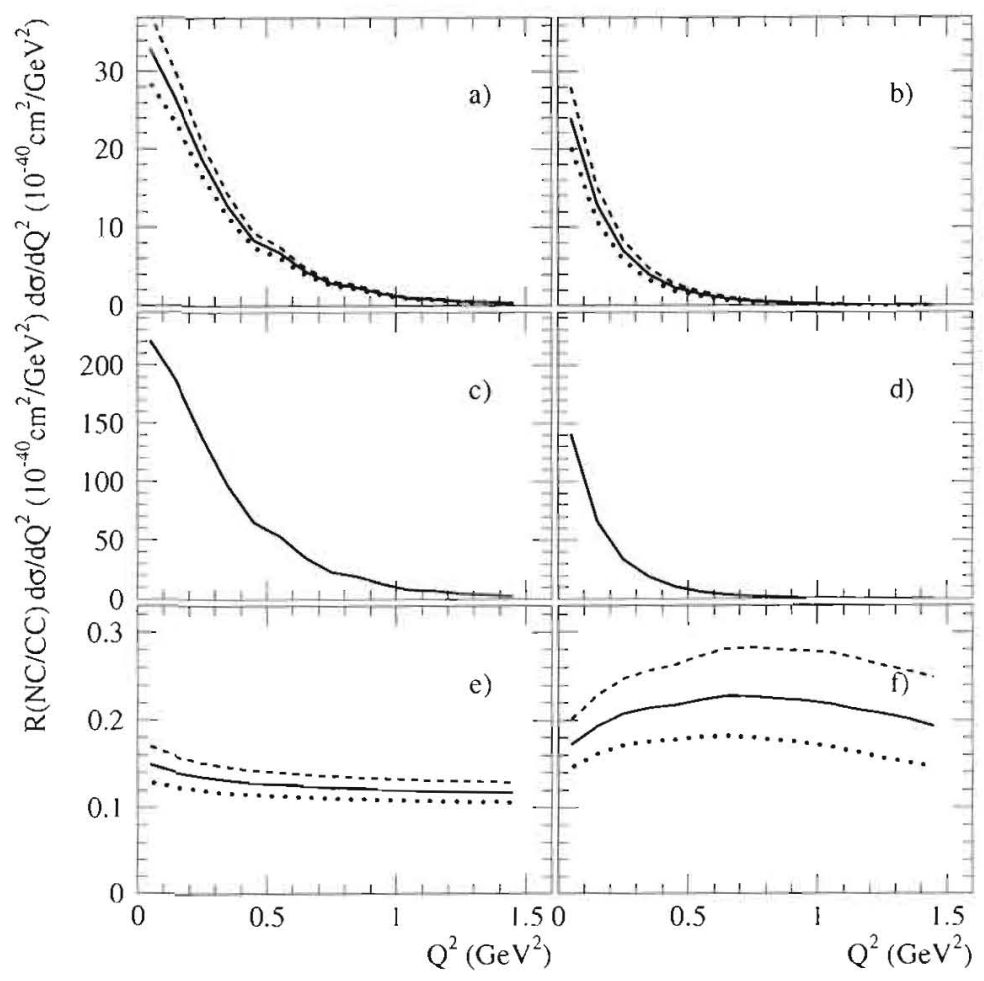

Figure 2.4: Flux-weighted differential cross sections and NC/CC ratios for both neutrino and anti-neutrino scattering as a function of $Q^{2}$. Plots (a)-(d) show the fluxweighted differential cross sections for (a) $\nu_{\mu} p \rightarrow \nu_{\mu} p$, (b) $\bar{\nu}_{\mu} p \rightarrow \overline{\nu_{\mu}} p$, (c) $\nu_{\mu} n \rightarrow \mu^{-} p$, (d) $\overline{\nu_{\mu}} p \rightarrow \mu^{+} n$. The NC/CC ratio for $\nu$ scattering is shown in (e), the NC/CC ratio for $\overline{\nu_{\mu}}$ scattering is shown in (f). These quantities are shown for $\Delta s=0$ (solid), $=-0.1$ (dashed), and $=+0.1$ (dotted). The CC processes do not depend upon $\Delta s$.

where $\frac{\sigma(R)}{R}$ is the relative error on the neutrino or anti-neutrino NC to CC ratio as measured in the experiment. The values for $\mathcal{S}$ as a function of $Q^{2}$ have been determined from plots like that shown in Fig. 2.5. The absolute value of $\mathcal{S}$ as a function of $Q^{2}$ for both neutrinos and anti-neutrinos is shown in Fig 2.6. Note that this plot shows only the sensitivity of the ratios to $\Delta s$. It does not take into account statistical errors or the uncertainty in extrapolating to $Q^{2}=0$.

Several conclusions may be made from these simple arguments based on the behavior of the $\mathrm{NC}$ and $\mathrm{CC}$ ratios:

- $R_{N C / C C}$ is quite sensitive to $\Delta s$. As can be seen from Fig. 2.6, the sensitivity at $Q^{2} \approx 0.25 \mathrm{GeV}^{2}$ is approximately 1.2. This implies that a measurement of 


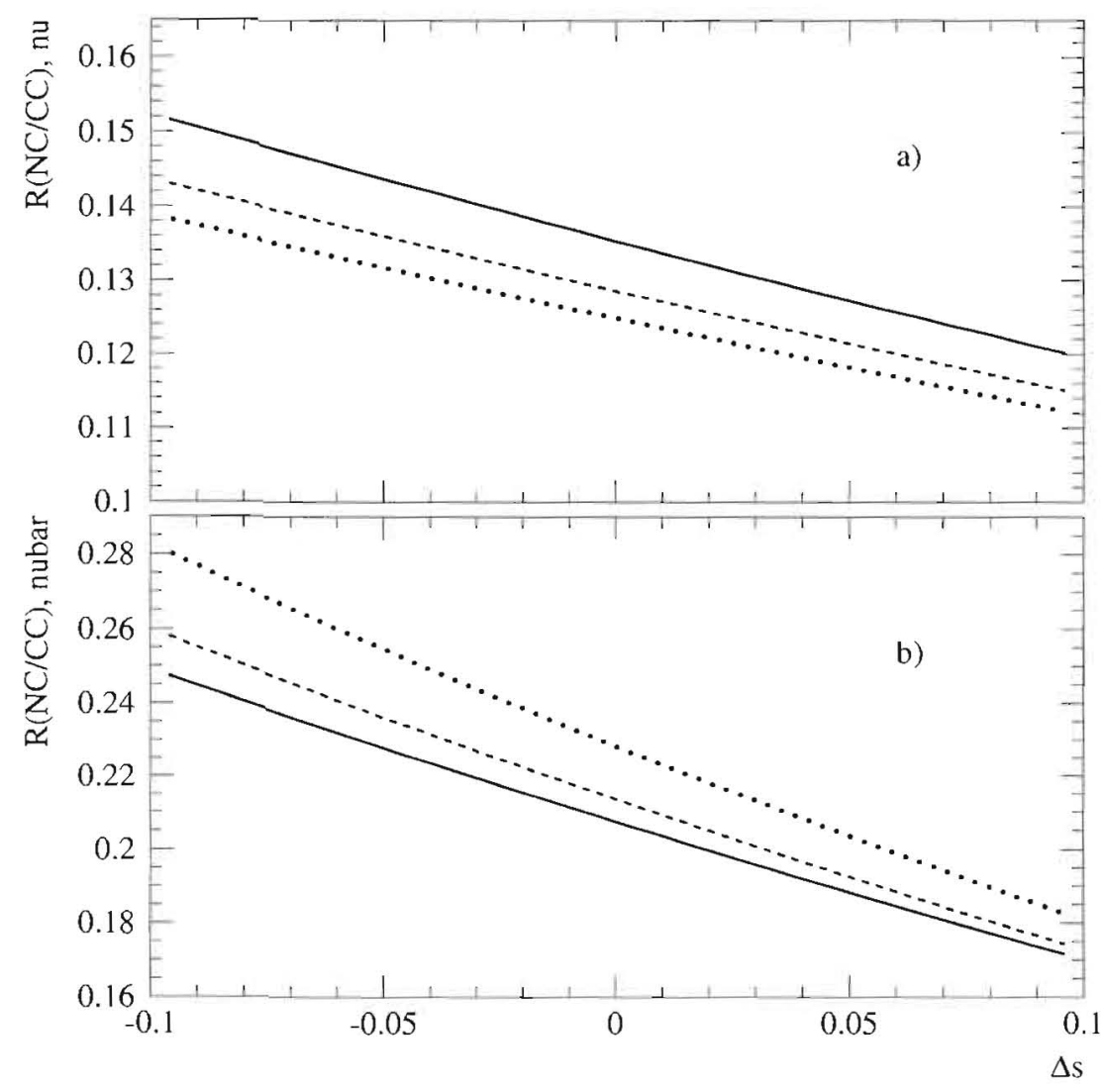

Figure 2.5: NC to $\mathrm{CC}$ ratio of flux-weighted cross sections for (a) neutrinos and (b) anti-neutrinos as a function of $\Delta s$ at $Q^{2}=0.25$ (solid), 0.45 (dashed), and 0.65 (dotted) $\mathrm{GeV}^{2}$.

$R_{N C / C C}$ with $5 \%$ relative error would enable an extraction of $\Delta s$ with an error of \pm 0.04 .

- $\bar{R}_{N C / C C}$ (the NC to CC ratio for anti-neutrinos) is even more sensitive to $\Delta s$. This is because the axial part of the cross section is a larger fraction of the total for anti-neutrino scattering. The sensitivity at $Q^{2} \approx 0.25 \mathrm{GeV}^{2}$ is approximately 1.9 for anti-neutrinos. This implies that a measurement of $\bar{R}_{N C / C C}$ of $5 \%$ would enable an extraction of $\Delta s$ with an error of \pm 0.03 . So the sensitivity is actually larger for anti-neutrinos than for neutrinos. In practice, the anti-neutrino measurement is more difficult due to lower anti-neutrino flux and reaction rates which will result in a larger error on $\bar{R}_{N C / C C}$. However, this will be offset somewhat by the larger sensitivity for anti-neutrinos. 


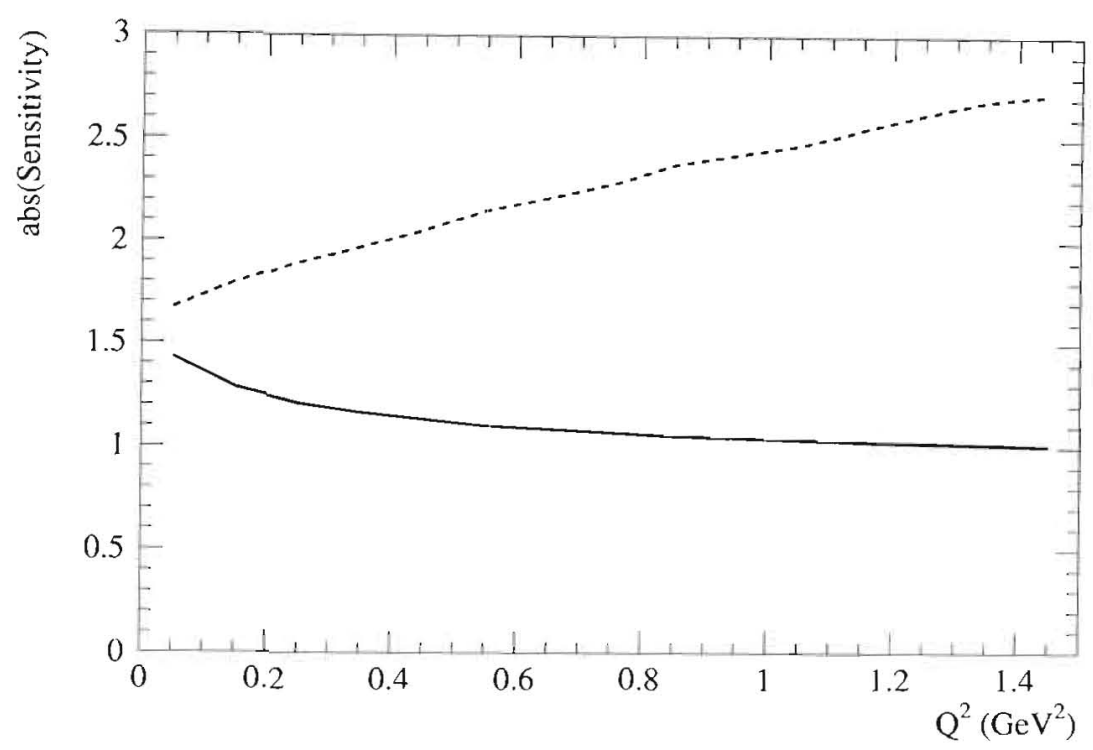

Figure 2.6: Absolute value of the $\mathrm{NC} / \mathrm{CC}$ sensitivity $\mathcal{S}$ to $\Delta s$ (defined in text) as a function of $Q^{2}$ for the neutrinos (solid) and anti-neutrinos (dashed). Note that this plot shows only the sensitivity of the ratios to $\Delta s$. It does not take into account statistical errors or the uncertainty in extrapolating to $Q^{2}=0$.

- The difference in sensitivities and the $Q^{2}$ dependence of $R_{N C / C C}$ and $\bar{R}_{N C / C C}$ is sizable as can be seen in Figures 2.4-2.6. A measurement for both neutrinos and anti-neutrinos will be a powerful constraint on systematic errors and the $Q^{2}$ dependence of the form factors.

\section{$\Delta s$ from $\nu N$ Scattering}

A simple interpretation of Figure 2.5 is that a $5 \%$ measurement of $R_{N C / C C}$ at $Q^{2} \approx$ $0.25 \mathrm{GeV}^{2}$ would enable an extraction of $\Delta s$ with an error of \pm 0.04 . In the actual measurement, of course, all of the data in our range of $Q^{2}$ will be used to extract $\Delta s$, and additional sources of error will demand consideration as well. These issues have been studied and quantified in Chapter 5: the added range in $Q^{2}\left(Q^{2}=0.2-1.0 \mathrm{GeV}^{2}\right)$ and improved statistics offset the additional sources of error. A measurement of $R_{N C / C C}$, as described in this Letter, over a range in $Q^{2}$ allows for a \pm 0.025 measurement of $\Delta s$.

Building on past experience, this measurement will improve on the results of BNL E734, the best neutrino-nucleon elastic scattering measurement to date, in the 
following ways:

- Collecting an order of magnitude more data to reduce the statistical errors;

- Performing the measurement at a lower $Q^{2}$ which reduces the systematic uncertainty in $\Delta s$ in extrapolating to $Q^{2}=0$;

- Reducing the background from other neutrino scattering processes, thereby reducing the systematic error on $R_{N C / C C}$;

- Designing the detector from the outset with the goal of measuring the NC to CC ratio, permitting additional systematic error reductions in detector efficiencies.

These error estimates are competitive with the claims from the DIS experiments. However, the result is not handicapped by assumptions of SU(3) flavor symmetry. Therefore, the experiment described in the Letter will provide the definitive statement on $\Delta s$.

\section{$\Delta s$ from a Global analysis of $\nu N, \bar{\nu} N$, and Parity Violating electron-nucleon Scattering Data.}

The preceding discussion assumes data from either a neutrino beam or an antineutrino beam only. We have demonstrated that a robust measurement of $\Delta s$ can be achieved with either of those data sets alone. The combination of the two data sets is a very important component in the measurement of $\Delta s$, so much so that a different analysis technique can be employed if both of those data sets are available. To make clear what this means, it is necessary to explain some of the details of how the data would be used in each case.

To determine $\Delta s$, it necessary to measure the strange axial form factor $G_{A}^{s}$ at a variety of $Q^{2}$ points, including points as close to $Q^{2}=0$ as possible, and then extrapolate to $Q^{2}=0$ to extract $\Delta s=G_{A}^{s}\left(Q^{2}=0\right)$. There are two other unknowns that come into play, namely the strange nucleon electric and magnetic form factors, $G_{E}^{s}$ and $G_{M}^{s}$. The data on the strange electromagnetic form factors will come from the program of parity-violating $\vec{e} N$ (PVeN) experiments: SAMPLE at MIT-Bates, PVA4 at Mainz, and $G^{0}$ and HAPPEX at Jefferson Lab. Because of complications arising from radiative corrections to the axial form factor that occur in PVeN experiments (but not in neutrino scattering), a complete measurement of $G_{E}^{s}$ and $G_{M}^{s}$ requires 
three observations: forward angle scattering on a proton target, and backward angle scattering on both proton and deuteron targets. Complete measurements of this type will only be available at four values of $Q^{2}: 0.1,0.3,0.5$, and $0.8 \mathrm{GeV}^{2}$. Only the three higher points are within the $Q^{2}$ range of FINeSSE. If FINeSSE only measures with neutrino beams, then we will have only these three points available to us for data on $G_{E}^{s}$ and $G_{M}^{s}$.

On the other hand, if FINeSSE makes two measurements of NC and CC scattering, using both neutrino and anti-neutrino beams, then only one additional piece of data would be needed from the PVeN measurements. It happens that in forward scattering PVeN measurements, the contribution from the axial term is strongly suppressed, and the complications mentioned above can be largely ignored. Then one may simple combine the numerous forward scattering PVeN data in the range $0.2<Q^{2}<0.8 \mathrm{GeV}^{2}$ with the FINeSSE data and extract $G_{A}^{s}$ at a much larger set of points that would have been possible otherwise. This will enable a much more robust extraction of $\Delta s$. This is essentially the same analysis technique used in Ref. [29] extended to lower $Q^{2}$ and using the better FINeSSE data. A complete simulation of the benefit of the anti-neutrino data to the $\Delta s$ measurement will be made available by the time of the PAC presentation of this Letter.

\subsection{Neutrino Cross Sections}

Reliable measurement of CC and NC neutrino cross sections at low energy $(0.1<$ $E_{\nu}<2 \mathrm{GeV}$ ) where the existing data are sparse to nonexistent, would be of great theoretical and practical value. From a practical standpoint, a quantitative understanding of these reactions is critical for both present and future neutrino oscillation experiments. In particular, improved cross section measurements would provide valuable input to atmospheric and accelerator-based neutrino oscillation searches which probe these same energies in order to reach their maximum sensitivity. From a theoreti-

cal standpoint, neutrino interactions on nuclear targets (having only been marginally studied in the past) would allow greater insight into nuclear dynamics, shadowing, and coherent pion production processes.

The motivation for measuring low energy neutrino cross sections is described here, while the sensitivity of our apparatus to these processes is outlined in Chapter 5. 


\subsubsection{Motivation for Improved Cross Section Measurements}

Current oscillation experiments model neutrino interactions in a regime that is poorly constrained by experimental data. Although accelerator-based neutrino beams have existed for over three decades, our primary knowledge of neutrino interactions at low energy comes almost entirely from bubble chamber measurements made decades ago at ANL, BNL, CERN, and FNAL, all of which were limited by low statistics and large neutrino flux systematics. In addition to these large uncertainties of typically $10-40 \%$, the experimental results often conflict and are difficult to interpret, mainly because of complications due to nuclear corrections and exclusive final state ambiguities. These data, while not as precise as DIS measurements, nonetheless are what presently constrain the Monte Carlo predictions used by many running neutrino experiments.

Improved knowledge of low energy neutrino cross sections will become increasingly important as experiments move from discovery to precision measurements of oscillation parameters. Consider the following examples:

- Present atmospheric constraints on $\Delta m_{23}^{2}$ and $\theta_{23}$ are already limited by flux and cross section systematics.

- Uncertainties on NC $\pi^{0}$ production cross sections currently restrict the ability to discriminate between $\nu_{\mu} \rightarrow \nu_{\tau}$ and $\nu_{\mu} \rightarrow \nu_{s}$ transitions in studies of enriched $\mathrm{NC}$ samples in atmospheric neutrino data.

- $\nu_{\mu} \rightarrow \nu_{e}$ appearance searches are limited by statistical and systematic errors related to background subtraction, most notably those associated with NC $\pi^{0}$ interactions where the final state photoconversion is mis-identified as an electron. Both the kinematics and rate of $\mathrm{NC} \pi^{0}$ production are less precisely known than most other reaction channels, because of the need to model resonant and coherent contributions in addition to potential feed-down from inelastic channels.

- $\nu_{\mu}$ disappearance measurements could profit from improved knowledge on both $\mathrm{QE}$ interactions on nuclear targets and CC single $\pi^{+}$production backgrounds.

More precise cross section measurements are not only important for ensuring the success of neutrino oscillation measurements, but are uniquely relevant for other searches. 
Resonant cross sections are a necessary input to $p \rightarrow \nu K^{+}$proton decay searches, because poorly measured atmospheric neutrino interactions such as $\nu_{\mu} n \rightarrow \mu^{-} K^{+} \Lambda$ and $\nu_{\mu} p \rightarrow \nu_{\mu} K^{+} \Lambda$ present significant backgrounds and hence large resultant systematics. In these regards, both present and future neutrino experiments could clearly benefit from improved knowledge of low energy neutrino cross sections.

\subsubsection{Present Understanding}

Figure 2.7 shows the contributing neutrino cross sections in the region of interest for atmospheric and terrestrial based neutrino oscillation experiments. At energies near $\sim 1 \mathrm{GeV}$, neutrino interactions include quasi-elastic (QE), resonant and coherent single pion production, and deep inelastic scattering (DIS) processes. Although DIS interactions have been measured with impressive precision $(<2 \%)$ at high energies, the cross sections of the various contributing processes at low energy are much less well-known (typically to $10-40 \%)$ and largely come from light target $\left(H_{2}, D_{2}\right)$ neutrino bubble chamber data. It is particularly challenging to measure and model neutrino interactions at low energies where there is substantial overlap between these various poorly-understood contributing processes. In this regard, the low energy regime is truly unique. The ability to successfully disentangle the various channels (QE, resonance, coherent, DIS, etc.) necessitates use of a fine-grained detector such as that being proposed here.

In contrast to many of these early low energy neutrino experiments, modern neutrino oscillation detectors employ heavy target materials (such as $\mathrm{C}, \mathrm{O}, \mathrm{Ar}, \mathrm{Fe}, \mathrm{Pb}$ ) in order to achieve their desired event rates. Complex targets add additional complications, such as the effects of Pauli blocking, Fermi motion of the target nucleons, and final state interactions (i.e. careful accounting for the fact that the outgoing hadron may re-interact before exiting the target nucleus). Final state effects (nuclear re-interactions, $\pi$ absorption, and charge exchange) often dominate; they can vary depending on the neutrino process, and their contributions certainly have not been disentangled experimentally. Nuclear effects significantly impact both the rate and kinematics of the neutrino reaction, as well as the observed final state event composition and multiplicity. Although nuclear effects have been studied extensively using muon and electron beams, no comparable effort has been made using neutrinos. Neutrino cross sections have been measured on nuclear targets in the past ${ }^{1}$, but these

\footnotetext{
${ }^{1}$ Gargamelle $\left(\mathrm{C}_{3} \mathrm{H}_{8} \mathrm{CF}_{3} \mathrm{Br}\right)$, SKAT $\left(\mathrm{C}_{3} \mathrm{H}_{8} \mathrm{CF}_{3} \mathrm{Br}\right)$, FNAL (Ne), CHARM and CHARM II (marble,
} 


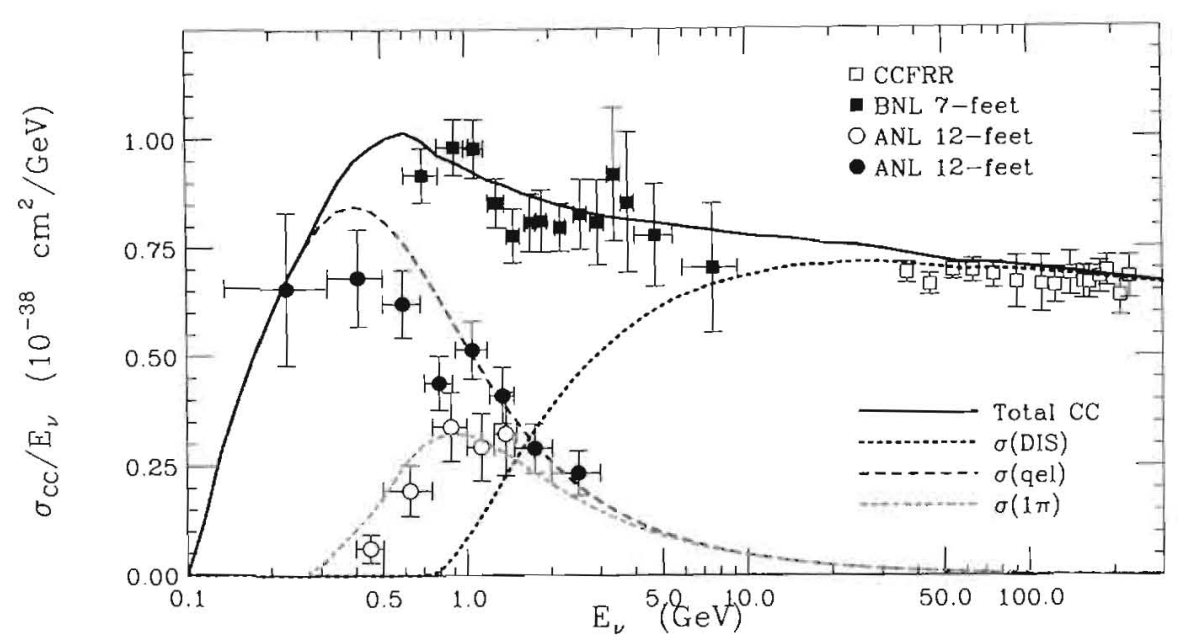

Figure 2.7: Charged current neutrino cross section contributions for an isoscalar target as a function of neutrino energy including quasi-elastic (dashed), single pion (dotdash), and deep inelastic scattering (dotted) processes. Plot is from reference [32].

experiments suffered from low statistics and typically published only free nucleon cross sections. By making dedicated, high statistics measurements of neutrino interaction cross sections on a scintillator-based target, this high precision, high statistics experiment could greatly improve the current experimental situation.

Several efforts are already underway to more precisely measure neutrino interactions on nuclei at low energy. Measurements of $\mathrm{NC} \pi^{0} / \mathrm{QE}$ and inelastic/QE event ratios have been recently performed in the $\mathrm{K} 2 \mathrm{~K}$ water Čerenkov and scintillator-based fine grain near detectors [33]. These measurements exhibit $\sim 10 \%$ accuracy based on samples of roughly 5,000-10,000 events [34]. MiniBooNE can additionally offer improved cross section constraints, with increased statistics over the $\mathrm{K} 2 \mathrm{~K}$ near detector ensemble. Such Čerenkov-based detection methods are nonetheless inherently limited in their capabilities. The rest of this section highlights the value of combining large event samples with fine-grained detection.

glass), and Serpukhov (Al) are several examples. 


\subsubsection{Prospects for Measuring Cross Sections: Some Exam- ples}

The following subsections outline prospects for several exclusive cross section measurements. This includes improved constraints on $\mathrm{NC} \pi^{0}$ production which would help improve the sensitivity of neutrino oscillation experiments looking for $\nu_{e}$ appearance, as well as important measurements of anti-neutrino cross sections.

\section{Neutral Current $\pi^{0}$ Production}

The dominant backgrounds to $\nu_{\mu} \rightarrow \nu_{e}$ appearance searches result from two principal sources: the intrinsic $\nu_{e}$ component in the beam and $\mathrm{NC} \pi^{0}$ production where the final state photoconversion is misclassified as an electron. Current experiments rely almost entirely on Monte Carlo simulations to estimate their $\pi^{0}$ backgrounds. Such simulations must model several mechanisms for producing a single $\pi^{0}$ : resonant and non-resonant production, coherent single pion production, and deep inelastic scattering in which additional hadrons are absorbed in the nuclear medium before being detected. The dominant means of single pion production at low energy arises through this first production mechanism: excitation of baryon resonances $(\Delta, N)$ that decay as:

$$
\begin{array}{ll}
\nu_{\mu} N \rightarrow l \quad N^{*} & \\
& N^{*} \rightarrow \pi N^{\prime} .
\end{array}
$$

There are seven such resonant neutrino reaction channels: three charged current and four neutral current:

$$
\begin{aligned}
& \nu_{\mu} p \rightarrow \mu^{-} p \pi^{+} \\
& \nu_{\mu} n \rightarrow \mu^{-} n \pi^{+} \nu_{\mu} n \pi^{+} \\
& \nu_{\mu} n \rightarrow \nu_{\mu} p \pi^{0} \\
& \nu_{\mu} n \rightarrow \mu^{-} p \pi^{0} \nu_{\mu} n n \pi^{0} \\
& \nu_{\mu} n \rightarrow \nu_{\mu} p \pi^{-}
\end{aligned}
$$

Traditionally, Monte Carlo simulations covering the low energy region have used theoretical calculations by Rein and Sehgal [35] to predict the rate and kinematics of 
neutrino resonance production. Such models are tuned to reproduce neutrino single pion data, but remain poorly constrained, because of the limited availability and large uncertainties in existing experimental data. As an example, Figure 2.8 shows the experimental constraints on the three $\mathrm{CC}$ resonant single pion production channels. Note that some of the data are conflicting and all of the data at low energy $\left(E_{\nu}<2 \mathrm{GeV}\right)$ were collected from light targets.
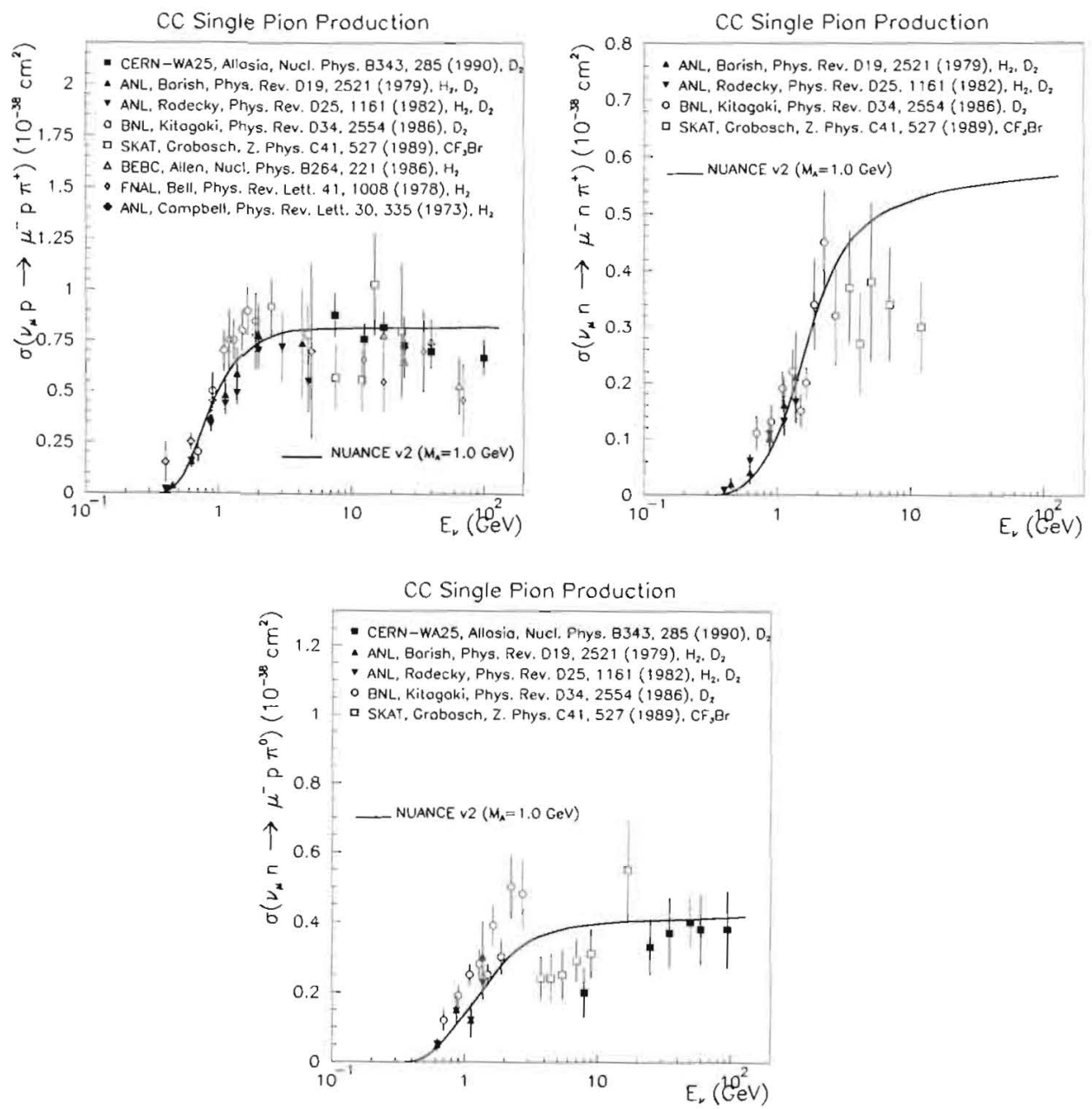

Figure 2.8: Experimental data on the three $\mathrm{CC}$ resonant single pion production cross sections $\sigma\left(\nu_{\mu} p \rightarrow \mu^{-} p \pi^{+}\right), \sigma\left(\nu_{\mu} n \rightarrow \mu^{-} n \pi^{+}\right)$, and $\sigma\left(\nu_{\mu} n \rightarrow \mu^{-} p \pi^{0}\right)$. Also shown in each case is the Rein and Sehgal-based prediction from the NUANCE Monte Carlo [36].

In addition to resonance production, neutrinos can also coherently produce single pion final states. In coherent interactions, neutrinos scatter off the entire nucleus 
rather than its individual constituents. Because of the negligible energy transfer to the target nucleus $(A)$, such processes distinctly produce a single forward-scattered pion. Like in the resonant case, both $\mathrm{NC}$ and $\mathrm{CC}$ processes are possible:

$$
\begin{aligned}
& \nu_{\mu} A \rightarrow \nu_{\mu} A \pi^{0} \\
& \nu_{\mu} A \rightarrow \mu^{-} A \pi^{+}
\end{aligned}
$$

Figure 2.9 shows the only available experimental measurements of resonant and coherent $\mathrm{NC} \pi^{0}$ absolute cross sections at low energy. No published measurements exist below $2 \mathrm{GeV}$ for either process. In the case of resonant $\mathrm{NC} \pi^{0}$ production, there are only two reported measurements, one from a recent re-analysis of Gargamelle bubble chamber data [37] and the other appearing as a footnote in an early spark chamber paper [38]. Data on coherent NC $\pi^{0}$ production are more copious, especially at higher energies, but at low energy, there are only two reported measurements. As a result, theoretical predictions for coherent rates below $2 \mathrm{GeV}$ vary widely. Almost all Monte Carlo simulations in current use base their predictions on Rein and Sehgal's original calculation [39] of coherent pion production cross sections and kinematics. More recent calculations [40] yield a factor of 2-20 less coherent pion production at, these energies than the earlier Rein and Sehgal predictions [39]. Because of the lack of low energy experimental data and the existence of several conflicting theoretical predictions, oscillation experiments typically assign a $100 \%$ uncertainty to coherent processes. This large uncertainty is especially important because coherent production may comprise up to $20 \%$ of the overall $\mathrm{NC} \pi^{0}$ rate. The ability to further constrain $\mathrm{NC} \pi^{0}$ production at low energies would thus be of great use in achieving increased sensitivity to $\nu_{\mu} \rightarrow \nu_{e}$ oscillations, and in placing more stringent limits on the oscillation of standard neutrinos to sterile states.

With its superior ability to identify and isolate $\pi^{0}$ interactions, this experiment could more precisely measure both resonant and coherent $\mathrm{NC} \pi^{0}$ production cross sections on carbon at these energies $\left(\left\langle E_{\nu}\right\rangle \sim 0.7 \mathrm{GeV}\right)$, thus providing an important constraint to accelerator- and atmospheric-based $\nu_{e}$ appearance experiments employing heavy nuclear targets. Such efforts also complement higher energy scintillator-based fine-grained analyses currently underway at the $\mathrm{K} 2 \mathrm{~K}$ near detector site $\left(\left\langle E_{\nu}\right\rangle \sim 1.3\right.$ $\mathrm{GeV})$ or being planned using the NuMI low energy beam $\left(\left\langle E_{\nu}\right\rangle \sim 10 \mathrm{GeV}\right)$. 

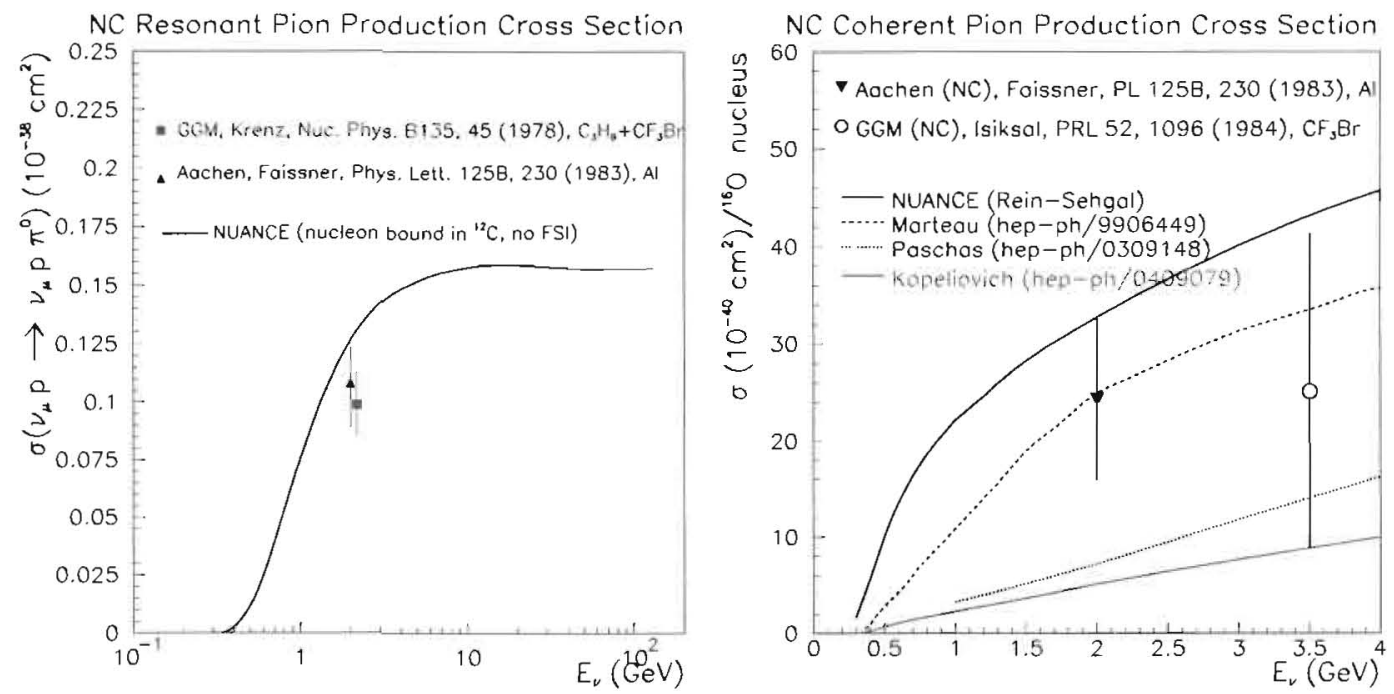

Figure 2.9: Resonant (left) and coherent (right) NC $\pi^{0}$ measurements at low energy. The NUANCE [36] curve, as plotted, does not include the effects of $\pi^{0}$ absorption in the target which would further reduce the predicted cross section. Also shown in the coherent case are the predictions from several more recent theoretical calculations [41].

\section{Anti-neutrino Cross Sections}

While there is not much published data on low energy neutrino cross sections, measurements of anti-neutrino cross sections are even more scarce. Figure 2.10 shows the existing experimental constraints on the anti-neutrino QE scattering cross section. All come from bubble chamber measurements that were made on a variety of targets decades ago. Combined, the entire QE data set from all experiments shown is under 2,000 events.

Additional anti-neutrino data on heavy nuclear targets are sorely needed so that interaction spectra and background rates for future anti-neutrino oscillation experiments can be estimated with confidence. Moreover, to ensure the robustness of future CP violation measurements, one would prefer to avoid relying heavily on Monte Carlo predictions and extrapolations into regions where no data exist. This experiment could be the first to produce high statistics, fine-grained $\overline{\nu_{\mu}}$ cross section measurements below $1 \mathrm{GeV}$, and hence anchor the theoretical predictions in this energy regime. 


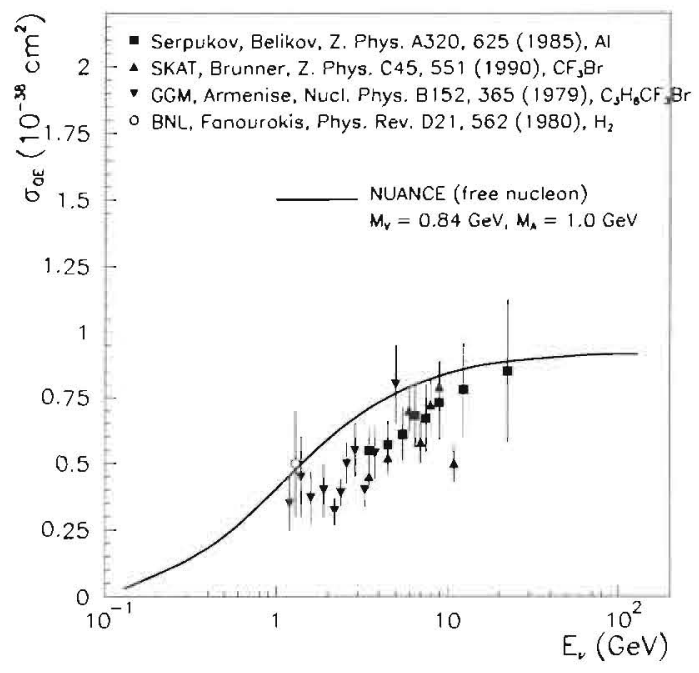

Figure 2.10: Experimental measurements of the $\overline{\nu_{\mu}} \mathrm{QE}$ cross section, $\sigma\left(\overline{\nu_{\mu}} p \rightarrow \mu^{+} n\right)$. Also shown is the free nucleon prediction from the NUANCE Monte Carlo [36].

\subsubsection{Global Importance}

The importance of low energy neutrino and anti-neutrino cross section measurements has been recognized in the wider neutrino community. The 2004 APS Multidisciplinary Study on the Future of Neutrino Physics cites as a high priority the need to better measure neutrino cross sections in the $\mathrm{MeV}$-to-GeV range [42]. With the measurements described here, this experiment would satisfy this need and thereby provide a major missing piece to the global neutrino program. 


\section{Chapter 3}

\section{The Neutrino Beam and Expected Event Rates}

\subsection{The Booster Neutrino Beam}

This section discusses the proton beam rate for the Booster Neutrino Beam and the beamline configuration ideal for the FINeSSE experimental run.

The August 6, 2004 memo "Prospects for the Booster Neutrino Beam" by Fermilab Director Mike Witherell states:

"Collaborations proposing experiments to run in the Booster neutrino beam in FY 2006 and beyond should plan their physics program on the basis of $1-2 \times 10^{20}$ protons on (the Booster Neutrino Beamline) target per year."

The beam requirements of the experiment are consistent with the upper end of this proton beam rate.

The FINeSSE run plan is 1 year of neutrino running followed by 2 years of antineutrino running. Thus, assuming $2 \times 10^{20}$ POT per year, the experiment would collect a total of $6 \times 10^{20}$ POT. With the detector located 80 meters from the target and with a $50 \mathrm{~m}$ decay length, 1 year of neutrino running is all that is necessary to collect the needed statistics to measure $\Delta s$ and neutrino cross sections in neutrino 
mode. Additional anti-neutrino running would significantly improve the $\Delta s$ determination as well as providing the largest, high-resolution sample to date for cross section measurements. The 2-year length of the anti-neutrino run was determined by balancing physics gain and run time. The total overall running time does not exceed 3 years or $6 \times 10^{20}$ POT. Details on this beamline configuration can be found in Chapter 6.

All other aspects of the beam line are identical to the present (March 2005) running of the MiniBooNE experiment (E898), except for the anti-neutrino running which requires a downtime of about a week to reverse the polarity of the horn.

\subsection{Booster Neutrino Beam Production}

\section{The Neutrino Flux}

The neutrino beam is produced by the $8 \mathrm{GeV}$ Fermilab Booster which currently feeds the MiniBooNE experiment. Protons from the Booster strike a $71 \mathrm{~cm}$ beryllium target inserted in a magnetic focusing horn. Protons arrive at this target in $1.6 \mu$ s long Booster spills. The timing structure within each spill delivers 842 ns wide bunches of beam, each separated by 18 ns. Secondary short-lived hadrons (primarily pions) produced in the target are focussed by the horn and enter a decay region. In normal MiniBooNE operation this decay region is $50 \mathrm{~m}$ long, followed by a beam absorber to stop hadrons and low energy muons.

The neutrino flux resulting from this design was simulated with the same tools used by the MiniBooNE collaboration [43]. The beam simulation utilizes GEANT 4 transport code [44], and the MiniBooNE JAM pion production model [43] which includes all beamline elements (horn, shielding, absorbers, etc.) and $\pi^{ \pm}, K^{ \pm}, K^{0}$ production from proton interactions on beryllium. To better reproduce the energy distribution of neutrino events observed in the MiniBooNE detector, pion spectra were input from a Sanford-Wang-based global fit [45] to pion production data in the relevant energy range in a procedure similar to that adopted by K2K. Figure 3.1 shows the resultant muon neutrino flux expected from a $50 \mathrm{~m}$ decay length beam produced at an $80 \mathrm{~m}$ FINeSSE detector site. In this configuration, $56.6 \times 10^{-9}$ muon neutrinos per POT per $\mathrm{cm}^{2}$ are anticipated with a mean energy of $\sim 700 \mathrm{MeV}$. The neutrino flux is roughly 70 times larger than that expected in a comparable volume at 


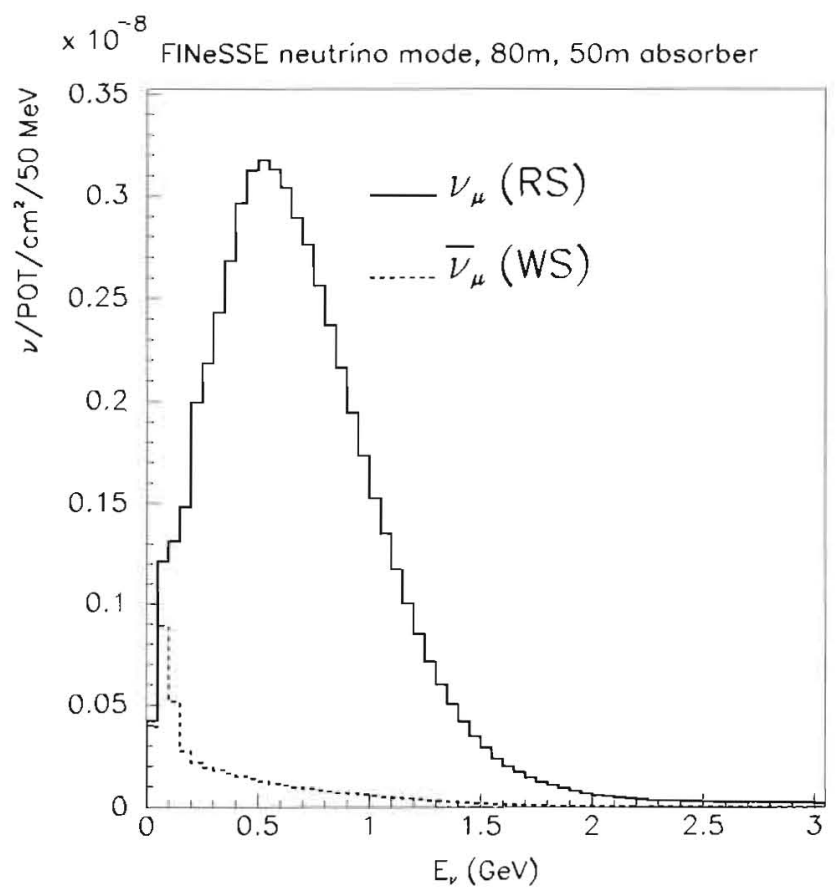

Figure 3.1: Expected neutrino mode $\nu_{\mu}$ (solid) and $\overline{\nu_{\mu}}$ (dashed) fluxes at an $80 \mathrm{~m}$ detector site assuming a $50 \mathrm{~m}$ decay length.

MiniBooNE. Antineutrinos comprise roughly $7 \%$ of the total flux in neutrino mode.

The polarity of the MiniBooNE horn can be reversed, allowing data to be taken in antineutrino mode. Figure 3.2 shows the resultant flux of antineutrinos expected at the FINeSSE detector. In this configuration, $33.2 \times 10^{-9}$ antineutrinos per POT per $\mathrm{cm}^{2}$ are anticipated with a mean energy of $\sim 600 \mathrm{MeV}$. The $\overline{\nu_{\mu}}$ flux in antineutrino running is a factor 1.7 lower than the $\nu_{\mu}$ flux expected in neutrino running. In this mode of running, there is also a larger expected "wrong-sign" background: neutrinos comprise roughly $16 \%$ of the total flux in antineutrino mode. This neutrino background in anti-neutrino running can be cosntrained to $7 \%$ of itself using the same techniques as developed for MiniBooNE Phase II running [46].

Better knowledge of the incoming neutrino beam flux enables more precise cross section measurements at both MiniBooNE and FINeSSE. The Booster neutrino flux will be much more precisely known than the fluxes reported in previous low energy neutrino cross section measurements well in advance of FINeSSE's commissioning. This improved knowledge comes from two sources: data from the Brookhaven E910 experiment [47] and from the CERN HARP experiment [48]. Analysis that is already underway of E910 proton-beryllium data taken at 6,12 , and $18 \mathrm{GeV}$ beam energies 


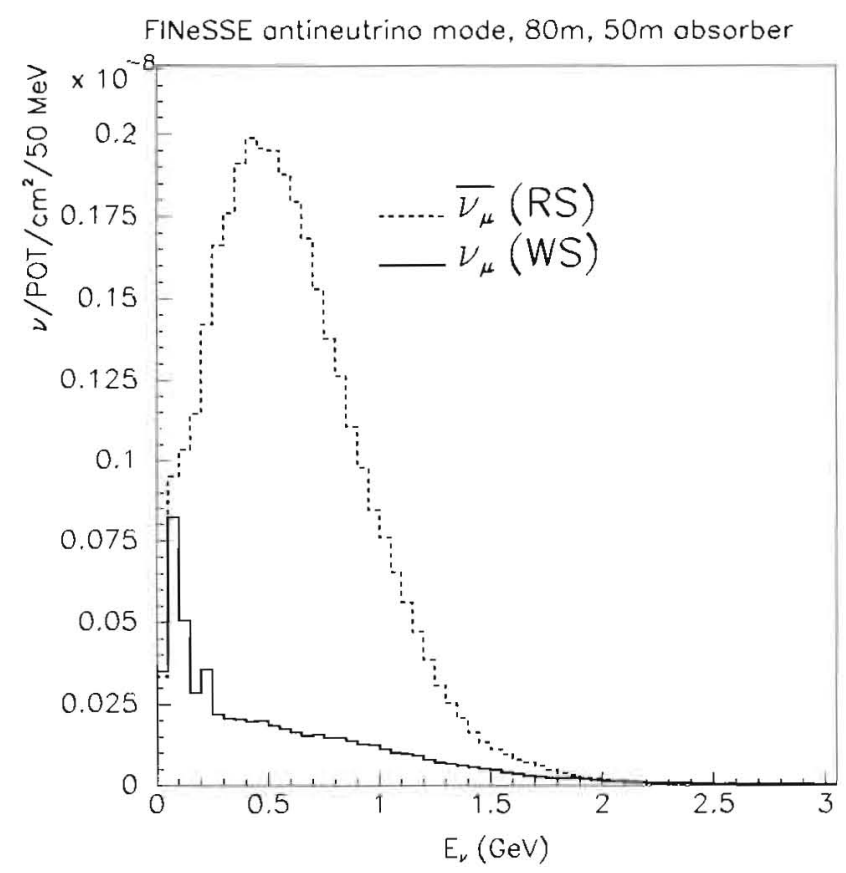

Figure 3.2: Expected antineutrino mode $\overline{\nu_{\mu}^{-}}$(dashed) and $\nu_{\mu}$ (solid) fluxes at an $80 \mathrm{~m}$ detector site assuming a $50 \mathrm{~m}$ decay length.

will be instrumental in verifying the extrapolation of the Sanford-Wang parametrization [45] to the $8 \mathrm{GeV}$ Booster beam energy. More importantly, HARP data taken at $8 \mathrm{GeV}$ on the Booster neutrino production target slugs will provide a tighter constraint on the flux. The high statistics HARP data will provide a statistical precision of $\sim 2 \%$ [49] on $\pi^{+}$production, which is the main source of muon neutrinos at both the FINeSSE and MiniBooNE detectors. Therefore, with these additional inputs, the overall muon neutrino flux at FINeSSE should be known to roughly 5\% [43].

\subsection{Event Rates}

The number of neutrino events expected in the FINeSSE Vertex Detector is calculated using the NUANCE Monte Carlo [36] to generate neutrino interactions on $\mathrm{CH}_{2}$. NUANCE is open-source code originally developed for simulating atmospheric neutrino interactions in the IMB detector. NUANCE has since been further developed and is now used by the K2K, Super-K, SNO, MiniBooNE, and MINERvA collaborations. The neutrino interaction cross sections in NUANCE have been extensively checked against published neutrino data and other available Monte Carlo event gen- 
erators. In addition, the full NUANCE simulation has been recently shown to provide a good description of events in both the MiniBooNE detector and $\mathrm{K} 2 \mathrm{~K}$ near detector ensemble.

For this specific use, NUANCE was modified to include the FINeSSE detector composition and geometry, as well as the incident neutrino flux at the $80 \mathrm{~m}$ detector site. Using the input neutrino flux distribution, NUANCE predicts event rates, kinematics, and final state particle topologies that can subsequently feed hit-level GEANT detector simulations, or, as in this case, simply estimate the type and number of neutrino interactions expected at FINeSSE.

Table 3.1 lists the expected neutrino mode event populations at the $80 \mathrm{~m}$ FINeSSE detector site assuming a $50 \mathrm{~m}$ decay length. The table provides the expected $\nu_{m u}$ rates per ton detector for $1 \times 10^{20}$ POT as well as the expected background from the $\overline{\nu_{\mu}}$ content in the beam. In all cases, the event rates have been normalized to the number of contained neutrino events observed in the MiniBooNE detector [43]. Roughly 1.6\% of the total neutrino events result from $\overline{\nu_{\mu}}$ interactions in the detector. The dominant contributions to the total event rate result from quasi-elastic and resonant processes: $42 \%$ of the $\nu_{\mu}$ events are CC quasi-elastic $\left(\nu_{\mu} n \rightarrow \mu^{-} p\right), 17 \%$ are NC elastic $\left(\nu_{\mu} N \rightarrow\right.$ $\left.\nu_{\mu} N ; N=n, p\right)$, and $33 \%$ resonant single pion production $\left(\nu_{\mu} N \rightarrow \mu^{-}\left(\nu_{\mu}\right) N \pi\right)$ channels.

A total of approximately 435,000 neutrino interactions can be expected at FINeSSE for the full request of $2 \times 10^{20} \mathrm{POT}$ in neutrino mode. This raw estimate assumes a 9 ton fiducial detector and 100\% detection/reconstruction efficiency.

Similarly, Table 3.2 lists anticipated antineutrino mode event populations. In this case, a larger fraction of events result from wrong-sign contamination: roughly $30 \%$ of the total events are $\nu_{\mu}$ interactions in the detector. In all, a total of approximately $170,000(75,000)$ antineutrino (neutrino) interactions can be expected at FINeSSE for the full request of $4 \times 10^{20} \mathrm{POT}$ in antineutrino mode. This raw estimate assumes a 9 ton fiducial detector and $100 \%$ detection/reconstruction efficiency. 


\begin{tabular}{|l|c|c|c|}
\hline Reaction & $\begin{array}{c}\nu_{\mu}(\mathrm{RS}) \\
10^{20} \mathrm{POT} \\
1 \text { ton }\end{array}$ & $\begin{array}{c}\overline{\nu_{\mu}}(\mathrm{WS}) \\
10^{20} \mathrm{POT} \\
1 \text { ton }\end{array}$ & $\begin{array}{c}\nu_{\mu}(\mathrm{RS}) \\
2 \times 10^{20} \mathrm{POT} \\
9 \text { ton }\end{array}$ \\
\hline \hline CC quasi-elastic & 10,107 & 181 & 181,930 \\
\hline NC elastic & 4,126 & 78 & 74,275 \\
\hline CC resonant $1 \pi^{+}$ & 4,990 & 0 & 89,827 \\
\hline CC resonant $1 \pi^{-}$ & 0 & 42 & 0 \\
\hline CC resonant $1 \pi^{0}$ & 928 & 13 & 16,704 \\
\hline NC resonant $1 \pi^{0}$ & 1,301 & 19 & 23,414 \\
\hline NC resonant $1 \pi^{+}$ & 458 & 8 & 8,237 \\
\hline NC resonant $1 \pi^{-}$ & 357 & 5 & 6,422 \\
\hline CC DIS & 253 & 2 & 4,550 \\
\hline NC DIS & 91 & 0 & 1,642 \\
\hline NC coherent $1 \pi^{0}$ & 365 & 14 & 6,566 \\
\hline CC coherent $1 \pi^{+}$ & 603 & 0 & 10,858 \\
\hline CC coherent $1 \pi^{-}$ & 0 & 24 & 0 \\
\hline other (multi- $\pi$, etc.) & 621 & 18 & 11,174 \\
\hline \hline total & 24,200 & 403 & 435,600 \\
\hline
\end{tabular}

Table 3.1: Number of neutrino mode events expected at $80 \mathrm{~m}$ with a $50 \mathrm{~m}$ decay length for $1 \times 10^{20}$ POT per ton detector and for the full requested FINeSSE running and detector (rightmost column). These predictions do not include final state effects in ${ }^{12} \mathrm{C}$ and assume $100 \%$ detection efficiency. 


\begin{tabular}{|c|c|c|c|}
\hline Reaction & $\begin{array}{c}\overline{\nu_{\mu}}(\overline{\mathrm{RS}}) \\
10^{20} \mathrm{POT} \\
1 \text { ton }\end{array}$ & $\begin{array}{c}\nu_{\mu}(\mathrm{WS}) \\
10^{20} \mathrm{POT} \\
1 \text { ton }\end{array}$ & $\begin{array}{c}\overline{\nu_{\mu}}(\mathrm{RS}) \\
4 \times 10^{20} \mathrm{POT} \\
9 \text { ton }\end{array}$ \\
\hline $\mathrm{CC}$ quasi-elastic & 2,219 & 787 & 79,892 \\
\hline NC elastic & 922 & 323 & 33,179 \\
\hline $\mathrm{CC}$ resonant $1 \pi^{+}$ & 0 & 470 & 0 \\
\hline $\mathrm{CC}$ resonant $1 \pi^{-}$ & 419 & 0 & 15,092 \\
\hline $\mathrm{CC}$ resonant $1 \pi^{0}$ & 130 & 93 & 4,666 \\
\hline $\mathrm{NC}$ resonant $1 \pi^{0}$ & 230 & 118 & 8,294 \\
\hline NC resonant $1 \pi^{+}$ & 83 & 43 & 2,996 \\
\hline NC resonant $1 \pi^{-}$ & 59 & 35 & 2,132 \\
\hline CC DIS & 3 & 30 & 116 \\
\hline NC DIS & 2 & 11 & 58 \\
\hline $\mathrm{NC}$ coherent $1 \pi^{0}$ & 184 & 30 & 6,624 \\
\hline $\mathrm{CC}$ coherent $1 \pi^{+}$ & 0 & 51 & 0 \\
\hline $\mathrm{CC}$ coherent $1 \pi^{-}$ & 298 & 0 & 10,714 \\
\hline other (multi- $\pi$, etc.) & 157 & 93 & 5,644 \\
\hline total & 4,706 & 2,086 & 169,402 \\
\hline
\end{tabular}

Table 3.2: Number of antineutrino mode events expected at $80 \mathrm{~m}$ with a $50 \mathrm{~m}$ decay length for $1 \times 10^{20}$ POT per ton detector and for the full requested FINeSSE running and detector (rightmost column). These predictions do not include final state effects in ${ }^{12} \mathrm{C}$ and assume $100 \%$ detection efficiency. 


\section{Chapter 4}

\section{The Detector}

A novel detection technique, described here, accurately measures shorttrack neutral current events, at all angles, particularly high angle, low $Q^{2}$ events most important for the $\Delta s$ analysis. Low energy threshold allows for good neutron tagging in the detector, crucial for the $\Delta s$ analysis. This fine-grained detector can also well measure single pion channels and quasielastic events.

The physics goals for this experiment require the ability to identify and track particles in $0.1-2.0 \mathrm{GeV}$ neutrino interactions. These reactions include neutralcurrent (NC) elastic scattering $\left(\nu_{\mu} p \rightarrow \nu_{\mu} p, \nu_{\mu} n \rightarrow \nu_{\mu} n\right)$, charged-current (CC) quasielastic scattering $\left(\nu_{\mu} n \rightarrow \mu^{-} p\right)$, as well as neutral- and charged-current production of pions $\left(\nu_{\mu} p \rightarrow \nu_{\mu} \pi X, \nu_{\mu} n \rightarrow \mu^{-} \pi X\right)$. It is necessary to measure both the charged and neutral hadrons and charged leptons in these reactions. The detector must also provide a large target volume in order to achieve the event rates needed to gather sufficiently large event samples.

\subsection{Detector Overview}

The detector will consist of a 13 ton (9 ton fiducial) liquid-scintillator, wavelengthshifting (WLS) fiber Vertex Detector with a downstream Muon Rangestack. The Vertex Detector will track particles as they emerge from the neutrino interaction vertex as well as measure the energy of the final state hadrons. The Muon Rangestack, 


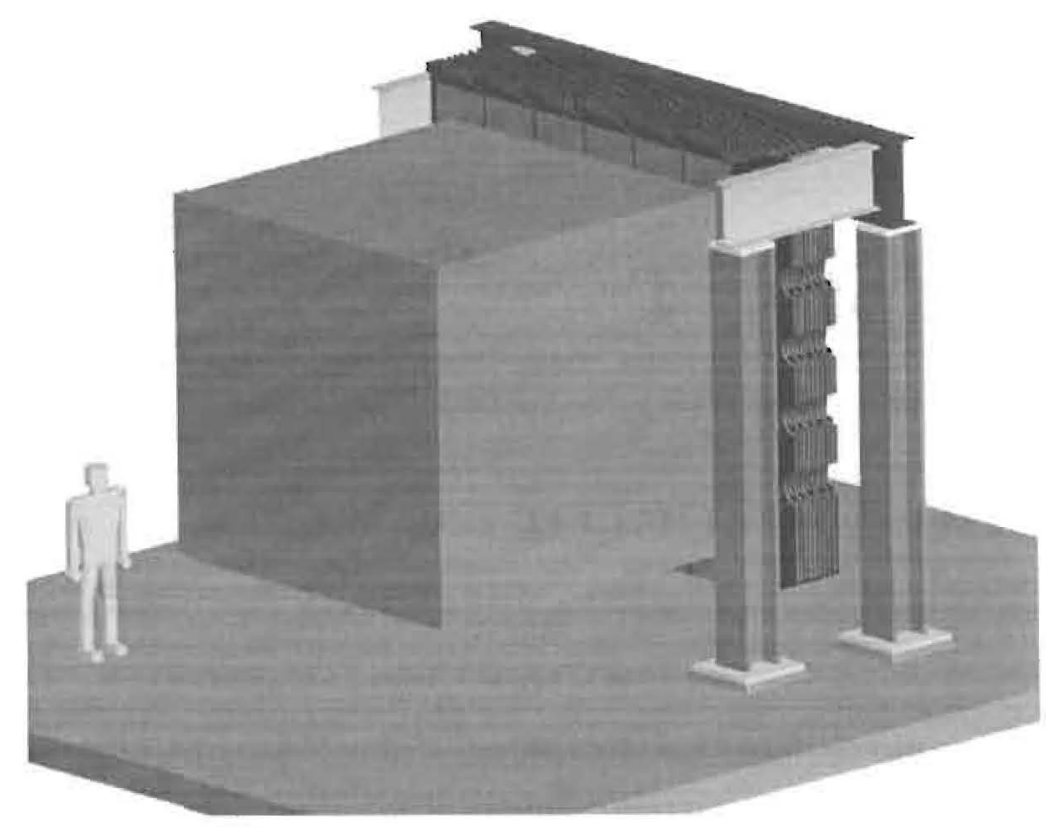

Figure 4.1: A schematic drawing of the detector. The cubic volume is the $(3.5 \mathrm{~m})^{3}$ Vertex Detector. The $(2.4 \mathrm{~m})^{3}$ signal region is surrounded by a veto, filled with liquid scintillator. The volume downstream consisting of multiple layers is the Muon Rangestack.

consisting of alternating planes of scintillator and iron, will range out and, therefore, measure the energy of the muons produced in CC reactions. This detector arrangement is shown in Figure 4.1. The cost estimate for this detector, with contingency, is $\$ 2.8 \mathrm{M}$.

An option to add an approximately 20 ton liquid Argon time projection chamber just upstream of the Vertex Detector is currently under study. This detector is not required to achieve the physics goals quoted in this Letter, but could greatly enhance the program in the future.

\subsection{The Vertex Detector}

The FINeSSE physics goals require a combination of high-efficiency tracking and good energy reconstruction for protons down to a kinetic energy of $100 \mathrm{MeV}$ as well as neutron tagging for final state neutrons. This necessitates a detector that has both 
fine segmentation and good calorimetry. As the name suggests, the Vertex Detector is the primary tracking detector, a fully instrumented neutrino target. The Vertex Detector precisely tracks the final-state particles in order to accurately determine the vertex location and event kinematics. The appropriate choice of scintillator oil and wavelength shifting fiber maximize the detector's tracking capability, as described in Section 4.2.1. The detector measures energy loss (" $d E / d x$ ") to help in separating proton and muon tracks as well as tagging final state neutrons when they capture via the process $n+p \rightarrow d+\gamma(2.2 \mathrm{MeV})$.

The Vertex Detector uses a technique called "scibath" which consists of WLS fibers immersed in a volume of liquid scintillator. The central liquid scintillator volume has dimensions of $2.4 \times 2.4 \times 2.4 \mathrm{~m}^{3}$. Light generated by ionizing particles traversing the scintillator is picked up by $1.5 \mathrm{~mm}$ diameter WLS fibers, submerged throughout the sensitive volume. There is no optical separation between the fibers. The fibers are mounted on a support frame, and are connected on one end to multianode photomultipliers, mounted to the outside of that frame. The fiber frame, photomultipliers, and associated electronics form a unit; this unit is immersed in the liquid scintillator, which is contained in a cubic tank, $3.5 \mathrm{~m}$ on a side. The volume between the fiber structure and the tank wall is instrumented with 2 layers of fibers with a spacing to be determined in order to detect and reconstruct charged particles entering and exiting from the tracking volume ("veto region"). The photomultiplier signals are processed in situ and transmitted on a data bus (Ethernet or similar) to the outside of the tank, thus minimizing the number of cables that penetrate the tank wall. A schematic drawing of the tracking detector is shown in Fig. 4.2. Cables penetrate the tank wall above the oil level to simplify leak-prevention.

Particle tracks can be reconstructed because the relative amount of light detected at the end of a fiber from a given source inside the detector is a known, continuous function of the distance between the source and the fiber.

The arrangement of the WLS fibers is shown schematically in Fig. 4.3. There are three sets of fibers, running parallel to the axes of a Cartesian coordinate system. Except for a rotation in space and an offset, the three fiber sets are identical, consisting of fibers that intercept the wall at the vertices of a quadrate grid. The distance between grid points is $30 \mathrm{~mm}$. Thus, the closest distance between any two fibers in the full assembly is $15 \mathrm{~mm}$. The resulting arrangement is invariant with respect to a rotation by $90^{\circ}$ about any major axis. For the given dimensions, there are a total of 


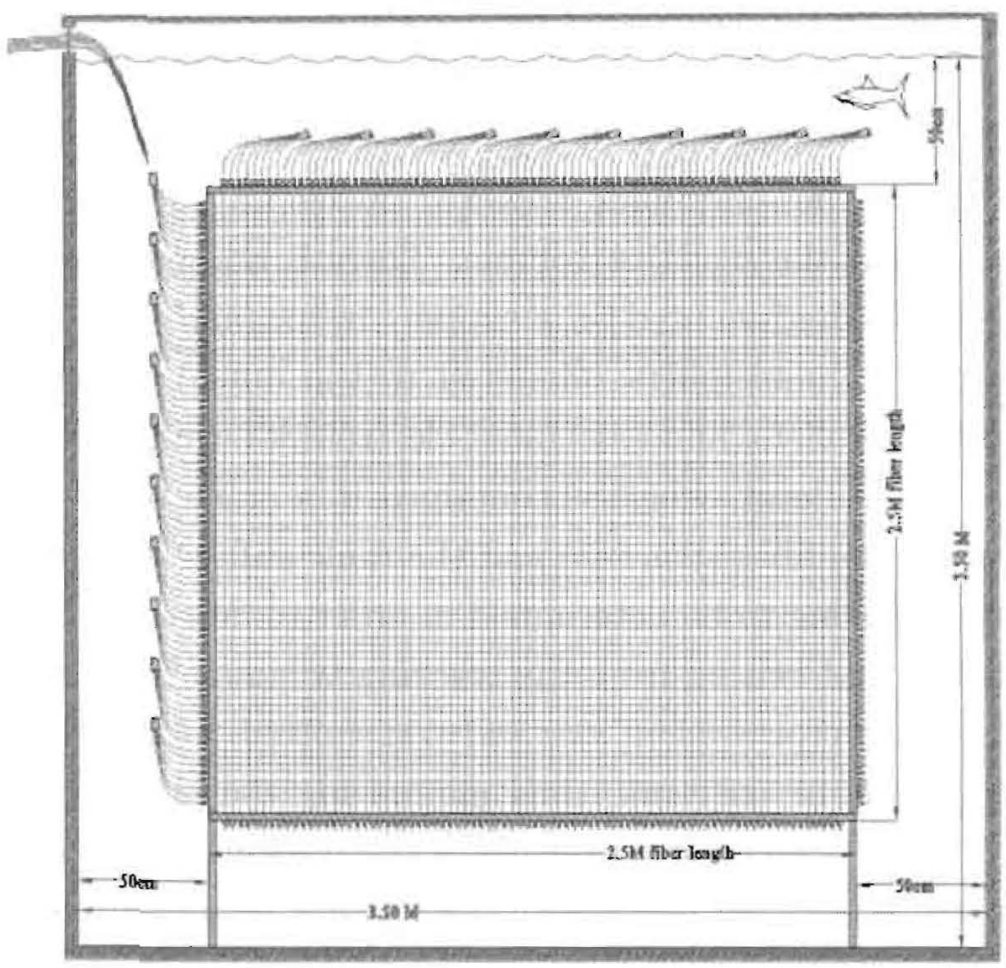

Figure 4.2: A schematic projection view of the Vertex Detector. 


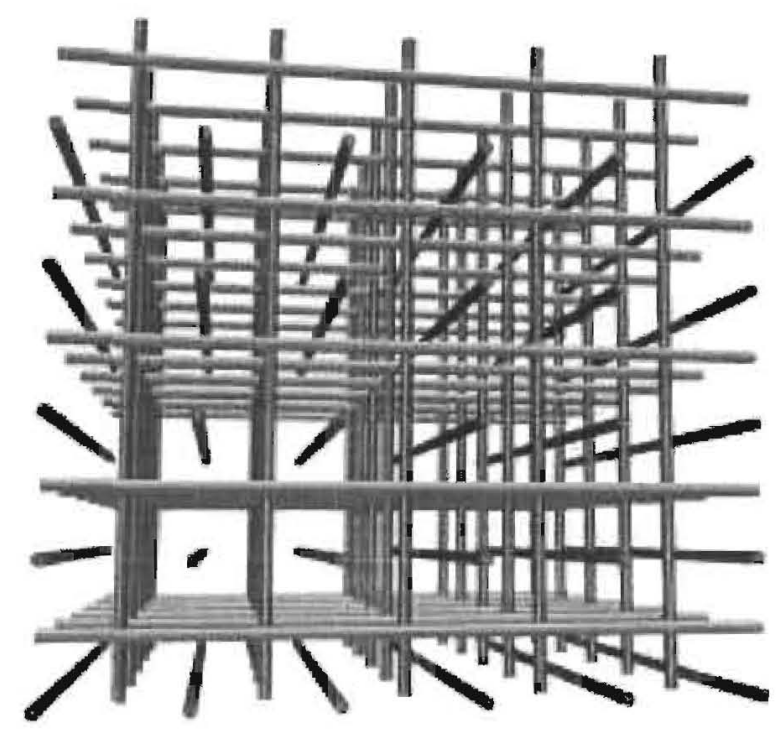

Figure 4.3: The geometrical arrangement of WLS fibers inside the Vertex Detector. The arrangement consists of three orthogonal sets of parallel fibers. The geometry is symmetric with respect to a rotation by $90^{\circ}$ about any of the three major axes.

$80 \times 80 \times 3=19200$ fibers.

This unique arrangement of fibers in the liquid scintillator volume offers several advantages over other detectors used in similar applications. There is no optical separation between the WLS fibers as is commonly employed in solid scintillator neutrino detectors [50]. This allows for greater sampling of the light produced by charged particle tracks which results in better tracking precision, especially for the short proton tracks that are of major interest in these physics measurements. A $100 \mathrm{MeV}$ proton travels only about $10 \mathrm{~cm}$ in liquid scintillator. In a solid scintillator bar detector, these tracks may only leave light in a few fibers which does not allow for good reconstruction of the track. However, in the "scibath" detector, these proton tracks will create light that is intercepted by several dozen fibers. This greater sampling allows for superior reconstruction, even for short tracks.

The arrangement of fibers in this detector with a grid parallel to each of the three major axes allows for three simultaneous track projections to be reconstructed. This is not possible in solid scintillator detectors or liquid scintillator detectors with optical separation between fibers. This technique results in better efficiency for tracks at $90^{\circ}$ to the beam direction. These tracks frequently produce light in a small number of fibers as they are parallel to one of the fiber directions. The scibath method avoids 
this problem as the tracks are at large angles with at least two of the three fiber directions.

\subsubsection{Prototype Tests}

In order to test the efficacy of this tracking method, a small prototype was constructed and tested with $200 \mathrm{MeV}$ protons at the Indiana University Cyclotron Facility. Tracking and light output were tested for several different scintillator oils and wave-length shifting fiber configurations.

\section{Prototype Setup}

The prototype device consisted of an anodized aluminum chamber of inside dimensions $30.0 \times 14.0 \times 12.6 \mathrm{~cm}^{3}$. A $6 \times 5$ grid of $1.5 \mathrm{~mm}$ diameter wavelength-shifting (WLS) fibers on $2 \mathrm{~cm}$ spacing penetrated the walls through an oil-tight seal with the long dimension of the fiber along the long $(30 \mathrm{~cm})$ dimension of the scintillator chamber. This arrangement of fibers allowed for tracking in one dimension. An assembly drawing of the detector is shown in Figure 4.4.

One end of the WLS fibers was routed to individual anodes of two $4 \times 4$ multianode Hamamatsu H8711 photomultiplier tubes (MAPMTs) [51]. The other end of the WLS fiber was terminated with a polished end. The entire assembly was made light-tight with metal covers.

The prototype detector was placed at the end of Beam Line I of the Radiation Effects Research Program (RERP) test station at IUCF [52]. A low-intensity ( $\approx$ $10 \mathrm{kHz}$ ), $200 \mathrm{MeV}$ proton beam with a $6 \times 6 \mathrm{~mm}^{2}$ profile (defined by two trigger scintillators) impinged on the detector. The detector could be moved vertically ( $y$ direction) and horizontally (x-direction). The box could also be rotated about the vertical $y$-axis (yaw) or the horizontal $x$-axis parallel to fiber direction (tilt). The PMT signals from each of the 30 fibers were integrated, digitized, and stored using CAMAC electronics upon a coincidence signal from the two $6 \times 6 \mathrm{~mm}^{2}$ trigger scintillators.

\section{Simulation}

In order to verify our understanding of the light production and transport processes in the prototype, a simulation of the device was created. The prototype was modeled 
using the GEANT detector simulation package [53]. The liquid scintillator and WLS fibers were constructed within the simulation. Scintillation light is modeled as an isotropic source along the proton track with a strength of 2500 photons per $1 \mathrm{MeV}$ deposited energy. Čerenkov light is also generated for particles above threshold, however, it is negligible compared to light from scintillation. The scintillation photons are tracked until they impinge on a fiber or are absorbed by the black walls of the detector. If a scintillation photon intersects a fiber it is considered detected with a $1 \%$ detection probability (the product of a $5 \%$ fiber capture efficiency with a $20 \%$ PMT quantum efficiency). In order to more efficiently simulate the data, the corrections were applied at the time of photon generation. By simulating individual photons, the statistics of individual photon collection are correctly handled. When comparing the simulation results to data, the overall light emission strength was adjusted slightly to match the data.

\section{Prototype Tests}

Two separate test sessions were conducted, each with a different liquid scintillator and different WLS fibers. The first session (data taken in September 2003) used Saint-Gobain [54] BCS517H liquid scintillator and BCF-91A WLS fibers. A second configuration was tested (data taken in May 2004) with Eljen [55] EJ-321L-NS scintillator and two different types of Saint-Gobain BCF-99 WLS fibers. The BCS517H liquid scintillator contains a wavelength shifter which results in an emission spectrum that peaks at $425 \mathrm{~nm}$. This scintillator has a long attenuation length for emitted light. In the second configuration, the scintillator contained no wavelength-shifting component and had a peak emission wavelength of $365 \mathrm{~nm}$ and a shorter attenuation length. This resulted in improved light localization for individual tracks. The scintillator properties for oil and WLS fibers used for each of these tests (labeled test session I and II) is summarized in Table 4.1.

In each of the test sessions I and II, a vertical scan of the detector was conducted by taking a series of runs with the beam location centered in the x-direction (see Fig. 4.4) and moved in the y-direction in $0.5 \mathrm{~cm}$ increments from the bottom to the top of the detector. This allowed for a determination of the light reduction with increasing distance from the fiber. For test session I, it also allowed for a calibration of each channel of the detector. 


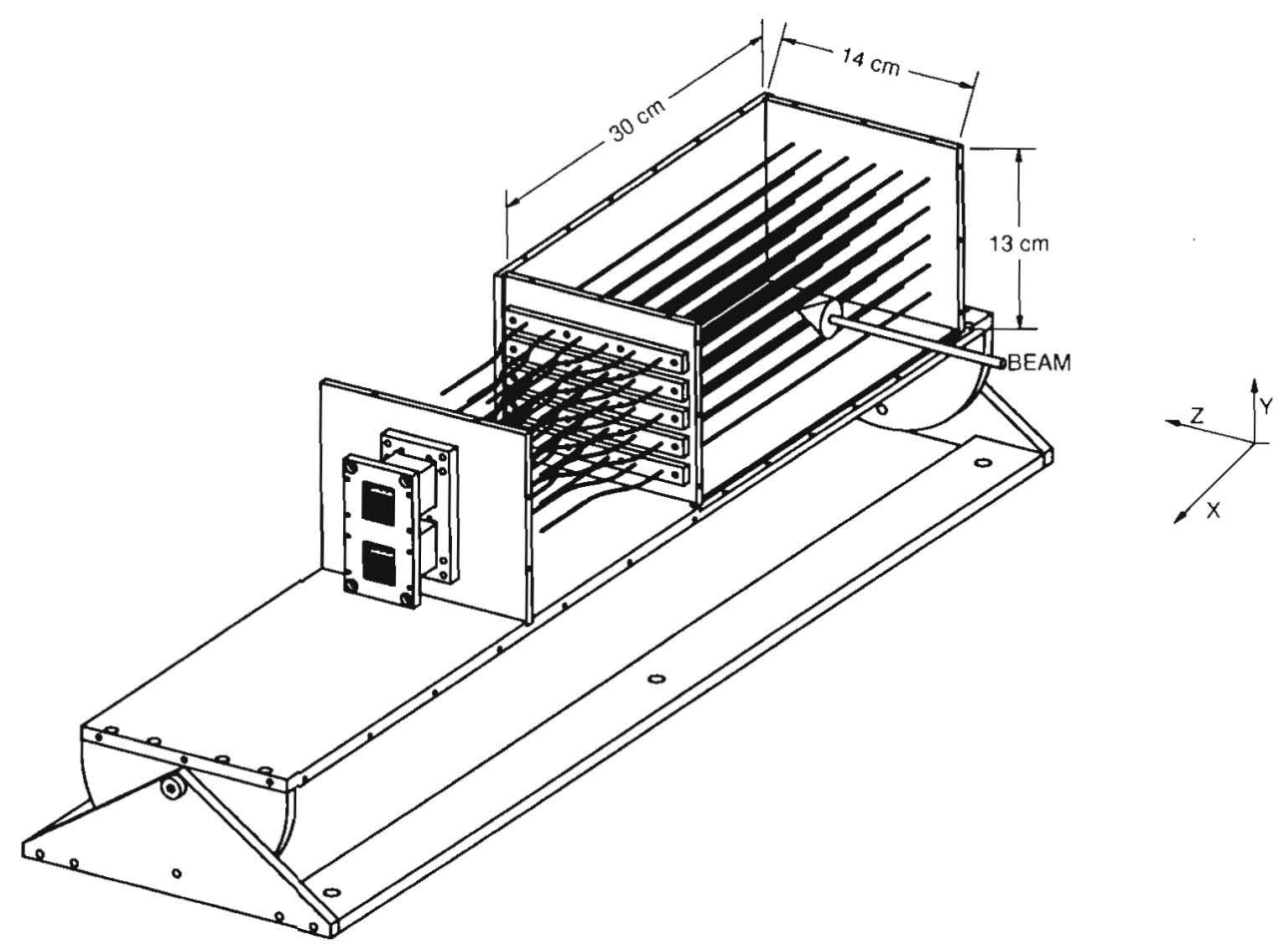

Figure 4.4: An assembly drawing of the scibath prototype detector. There are 5 rows of 6 fibers for a total of 30 wavelength-shifting fibers. The beam was incident along the z-axis.

\begin{tabular}{|c|c|c|c|c|c|c|}
\hline \multirow[b]{2}{*}{$\begin{array}{c}\text { test } \\
\text { session }\end{array}$} & \multirow[b]{2}{*}{$\begin{array}{l}\text { test } \\
\text { date }\end{array}$} & \multicolumn{2}{|c|}{ scintillator } & \multicolumn{3}{|c|}{ WLS fiber } \\
\hline & & type & $\begin{array}{c}\text { emission } \\
\text { peak }(\mathrm{nm})\end{array}$ & type & $\begin{array}{l}\text { absorption } \\
\text { peak (nm) }\end{array}$ & $\begin{array}{c}\text { emission } \\
\text { peak }(\mathrm{nm})\end{array}$ \\
\hline I & $09 / 03$ & BCS517H & 425 & BCF-91A (BG) & 420 & 494 \\
\hline II & $05 / 04$ & EJ-321L-NS & 365 & BCF-99 (UB) & 345 & 435 \\
\hline$"$ & $"$ & $"$ & $"$ & BCF-99 (UG) & 350 & 530 \\
\hline
\end{tabular}

Table 4.1: Summary of the scintillator and wavelength-shifting fibers used in the two prototype test sessions. 


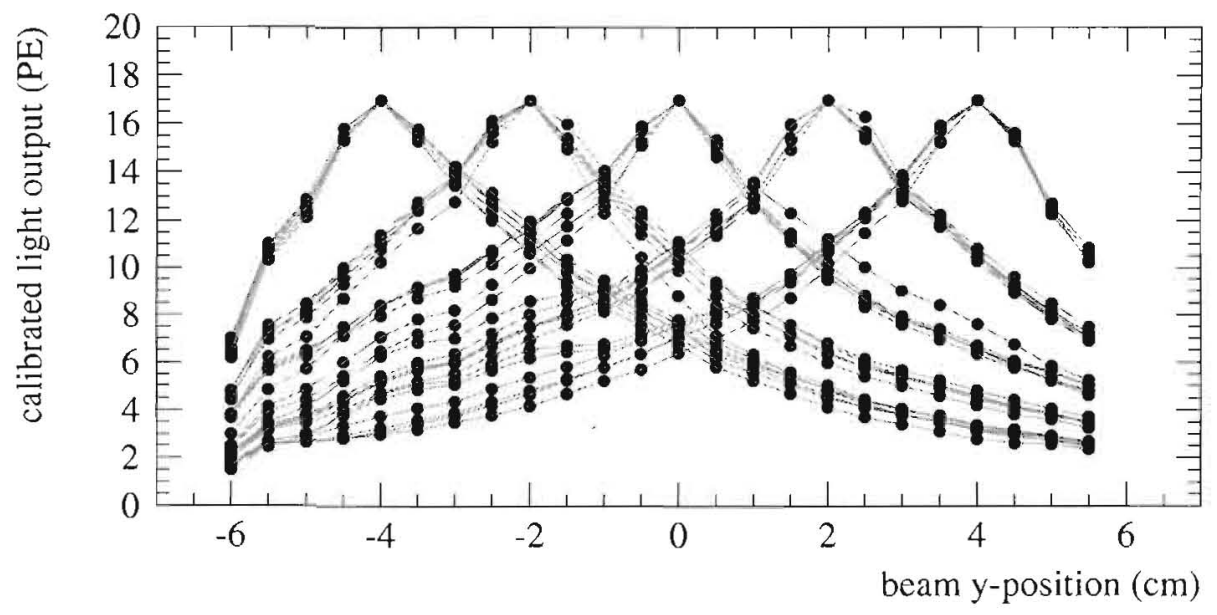

Figure 4.5: Light output from each of the 30 BG fibers taken during the vertical scan in test session I. The gain of one channel was absolutely normalized with a calibrated light source. The 5 peaks at the same beam y-position correspond to the 5 rows of 6 fibers each on a $2 \mathrm{~cm}$ spacing.

\section{Light Output}

The measured light amplitude from each of the 5 rows of 6 fibers, as a function of beam distance from a fiber, is shown in Figures 4.5 and 4.6 for test sessions I and II respectively. The maximum value of the light output of each fiber (when the beam was centered on a fiber row), is a measure of the light output for "near" proton tracks. This yielded $17 \pm 2$ photoelectrons for test session I with BG fibers. For test session II, the average of each fiber maximum yielded $17 \pm 2$ photoelectrons for UB fibers and $7 \pm 1$ photoelectrons for UG fibers.

These light output values can be compared to an prediction using the known scintillator yields together with the simulation program. The simulation accounts for the geometric acceptance of the fiber to photons from a "near" proton track. The 5\% fiber capture efficiency and 20\% PMT quantum efficiency are taken into account by reducing the generated scintillation yield from 2500 photons/MeV to 25 photons/MeV. In order to match the test session I data using BG fibers, the predicted light output from simulation had to be scaled by a factor of 0.8 . This implies an effective scintillation strength of 20 photons/MeV. If the known fiber capture efficiency (5\%) and PMT quantum efficiency at $494 \mathrm{~nm}(12 \%)$ is taken into account, the scintillation yield is 3300 photons/MeV. The quoted yield from Saint-Gobain BC517H 


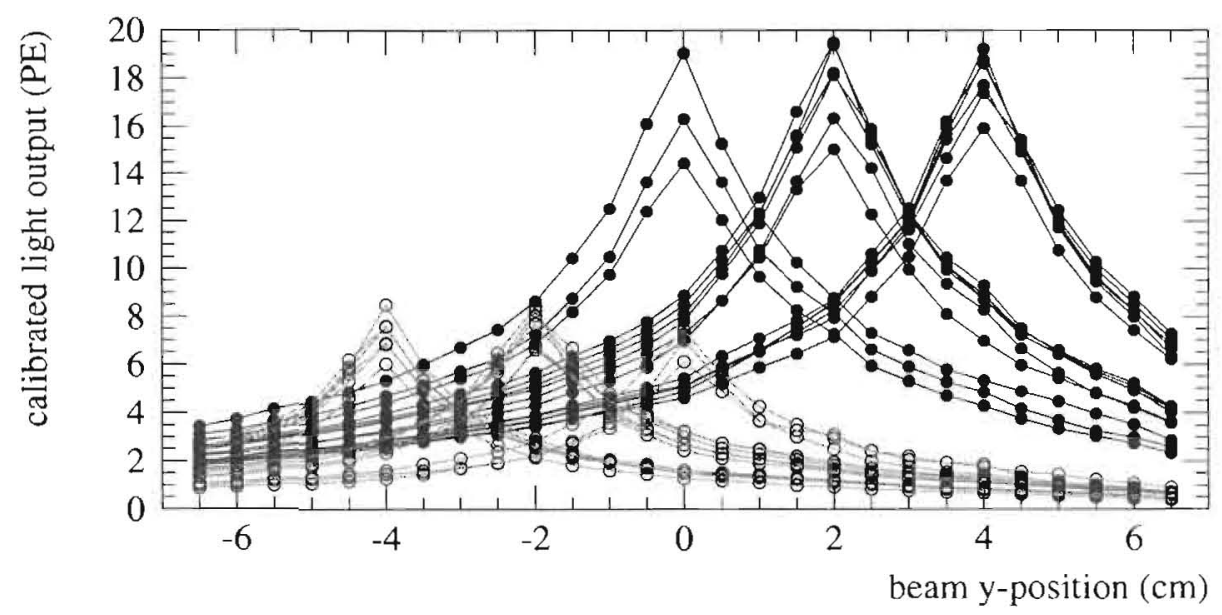

Figure 4.6: Results from vertical beam scan with 15 UB fibers (closed points) and 15 UG fibers (open points). The gain of each channel was absolutely normalized with a calibrated light source. The 5 peaks at the same beam y-position correspond to the 5 rows of 6 fibers each on a $2 \mathrm{~cm}$ spacing.

is $52 \%$ that of anthracene [54] or 8840 photons/MeV. This difference is likely due to the lower average quantum efficiency of the PMT over the fiber emission spectrum. In addition, other effects may contribute to the difference such as an inefficiency in the wavelength-shifting process, an imperfect collection of the light at the PMT interface, and optical effects at the scintillator fiber interface. The scintillation yield was also extracted for the test session II scintillator-fiber combinations. The results are summarized in Table 4.2 .

The light output from the UG fibers is substantially lower than the other two fiber types predominantly because of the lower PMT quantum efficiency at the peak fiber emission wavelength. In addition, the UG fiber absorption spectrum extends to lower wavelengths where the light attenuation length is small.

As is evident from Table 4.2, the measured scintillation yield is lower than expected by a factor of 3-4 for each of the three fiber-scintillator combinations. This is likely due to additional inefficiencies in the light transport processes. However, the measured light output is quite adequate for a a detector of this type, especially with the $B G$ and UB fibers. 


\begin{tabular}{|c|c|c|c|c|}
\hline $\begin{array}{c}\text { test } \\
\text { session }\end{array}$ & $\begin{array}{c}\text { fiber } \\
\text { type }\end{array}$ & $\begin{array}{c}\text { measured light } \\
\text { output (PE) }\end{array}$ & $\begin{array}{c}\text { meas. scint. } \\
\text { yield (PE/MeV) }\end{array}$ & $\begin{array}{c}\text { predicted scint. } \\
\text { yield (PE/MeV) }\end{array}$ \\
\hline I & BCF-91A (BG) & $17 \pm 2$ & 3300 & 8840 \\
II & BCF-99 (UB) & $17 \pm 2$ & 2000 & 6630 \\
II & BCF-99 (UG) & $7 \pm 1$ & 1500 & 6630 \\
\hline
\end{tabular}

Table 4.2: Summary of absolute light output for the scintillator/fiber combinations tested.

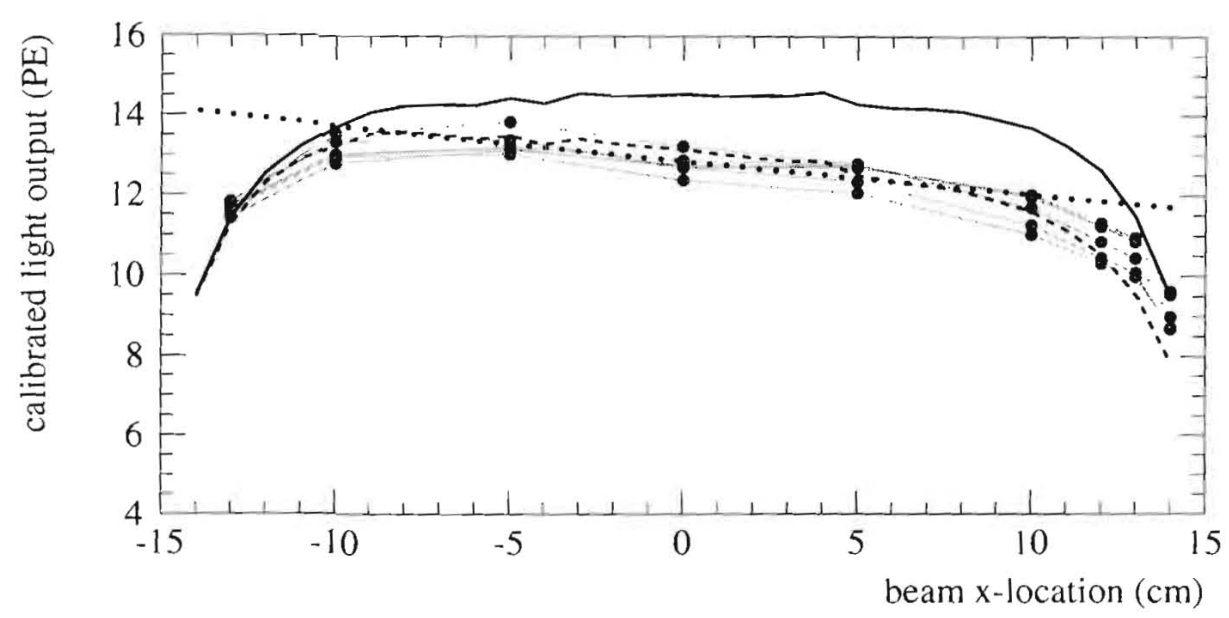

Figure 4.7: Data from the five fibers in the central row (closed data points) in a horizontal scan of the detector conducted in test session I. The solid line shows the simulation results with no attenuation, the dashed line shows these results with the addition of a $150 \mathrm{~cm}$ attenuation in the fiber. The dotted line shows the effect of a $150 \mathrm{~cm}$ attenuation length alone, arbitrarily normalized.

\section{Horizontal Scan}

To check the behavior of the detector as the track position changed along the length of the fiber, the beam was scanned along the device in the $\mathrm{x}$-direction at a $\mathrm{y}$-location of $1 \mathrm{~cm}$ ( $1 \mathrm{~cm}$ above the central row of fibers). The data, shown in Figure 4.7, behaves as expected from the simulation. They show a slow decrease at larger $\mathrm{x}$ positions (farther from PMTs) consistent with the attenuation length of the fiber $(\approx 1.5 \mathrm{~m})$. They also show a decrease at both ends of the detector due absorption of some scintillation light by the detector end-plates. As can be seen in Fig. 4.7, the simulation reproduces this effect adequately. 


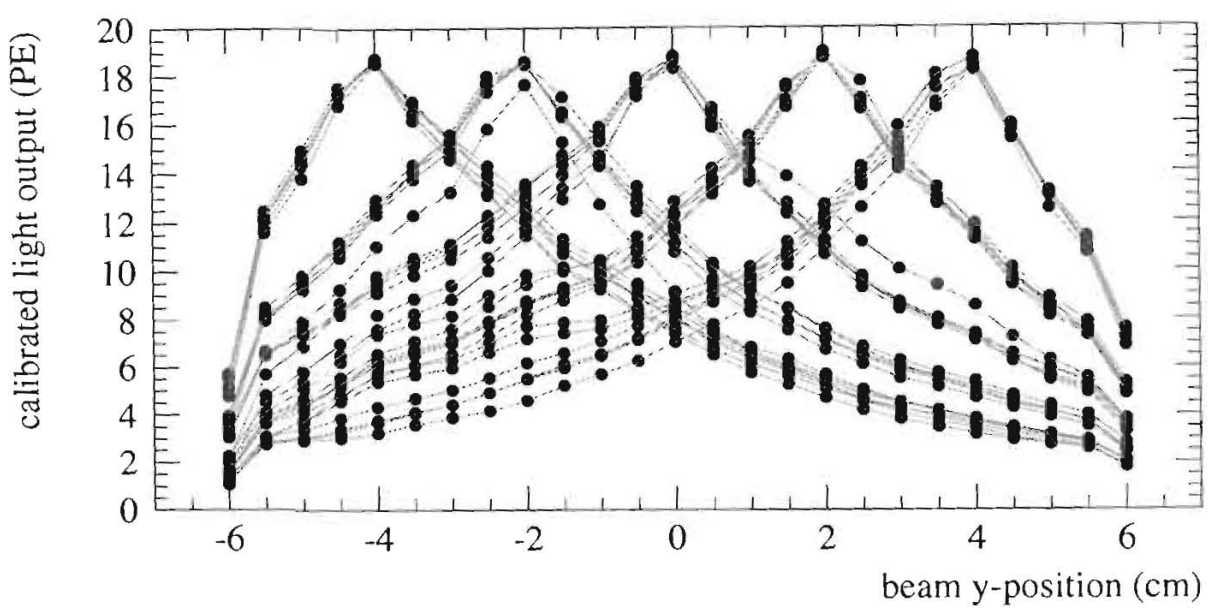

Figure 4.8: Results from a vertical scan across $30 \mathrm{BG}$ fibers with the prototype detector rotated around the vertical axis by $27^{\circ}$ (yaw). The 5 peaks at the same beam y-position correspond to the 5 rows of 6 fibers each on a $2 \mathrm{~cm}$ spacing. The increase in maximum gain from each row as compared to Figure 4.5 corresponds to the increased proton track-length.

\section{Yaw}

The response of the detector to tracks at oblique angles to the fibers was tested by rotating the detector (yaw) around the vertical y-axis by $27^{\circ}$ (see Fig. 4.4). A vertical scan was performed with the detector in this orientation. The data for all fibers is shown in Figure 4.8, and should be compared to the data in Figure 4.5. The yaw data shows an overall increase in light as would be expected due a longer track length near the fiber. The average of the maxima for all fibers is 18.6 photoelectrons. This is a factor of 1.09 higher than the data shown in Fig. 4.5 where the data yielded 17.0 photoelectrons for perpendicular tracks. This is consistent with the expected value since the light output should increase with the track-length as the geometrical factor, $1 / \cos \left(27^{\circ}\right)=1.12$.

\section{Tracking}

Ultimately, this detector technique is designed to provide 3-dimensional tracks formed from 2-dimensional projections in the $X Z, Y Z$, and $X Y$ planes. In this prototype, the fibers were oriented along the $x$-axis only and, therefore, measured the proton 
tracks in the $Y Z$ plane only (see Figure 4.4). It is possible to form tracks because the light amplitude as a function of distance is known. If this function is inverted, a measurement of the light amplitude from each fiber determines the distance to the track. The coordinates from each fiber are combined with this distance to form a particle track.

The dependence of the light amplitude was parameterized as,

$$
L=\frac{L_{0}}{1+r / s}
$$

where $L$ is the measured light output, $r$ is the fiber to track distance of closest approach, $L_{0}$ is a amplitude parameter and $s$ parameterizes the light fall off. For test session I, it was determined that $L_{0}=17.0$ photoelectrons and $s=2.86 \mathrm{~cm}$ provided an adequate description of the data. The fiber-track distance can then be determined from the light amplitude,

$$
r=s\left(\frac{L_{0}}{L}-1\right) \text {. }
$$

Tracks are formed by minimizing, for each event, the least squares sum,

$$
X^{2}=\sum_{i=1}^{n} \frac{\left(r_{i}-r_{\mathrm{fit}}\right)^{2}}{\sigma_{i}^{2}},
$$

over $n$ fibers. The error on the distance obtained from each fiber, $\sigma_{i}$, is calculated by standard propagation of the error on the light measurement from Equation 4.2. The fit track was parameterized with the form $y=a x+b$, where $a$ is the $\mathrm{y}$-slope and $b$ is the $y$-intercept.

The data from test session I was subjected to this procedure. The resulting distributions of $y$-intercept and $y$-slope values obtained from a run with the beam centered on the detector are plotted in Figure 4.9. As can be seen in the figure, the distributions are centered around zero, indicating no bias in the y-direction. The resolutions were extracted from a Gaussian fit to each distribution after subtracting the contribution due to the $6 \mathrm{~mm}$ square trigger scintillators. This contribution is $0.6 \mathrm{~cm} / \sqrt{(12)}=0.17 \mathrm{~cm}$ and was subtracted in quadrature from the total width of the distribution. This procedure yielded a position resolution of $0.44 \mathrm{~cm}$ and angular resolution of $5.6^{\circ}$.

The track fitting procedure was repeated for the vertical scan data and results in a reconstructed $y$-intercept and $y$-slope for a range of true $y$-intercept positions. These results are plotted in Figure 4.10. Note that the correct value for $y$-intercept 

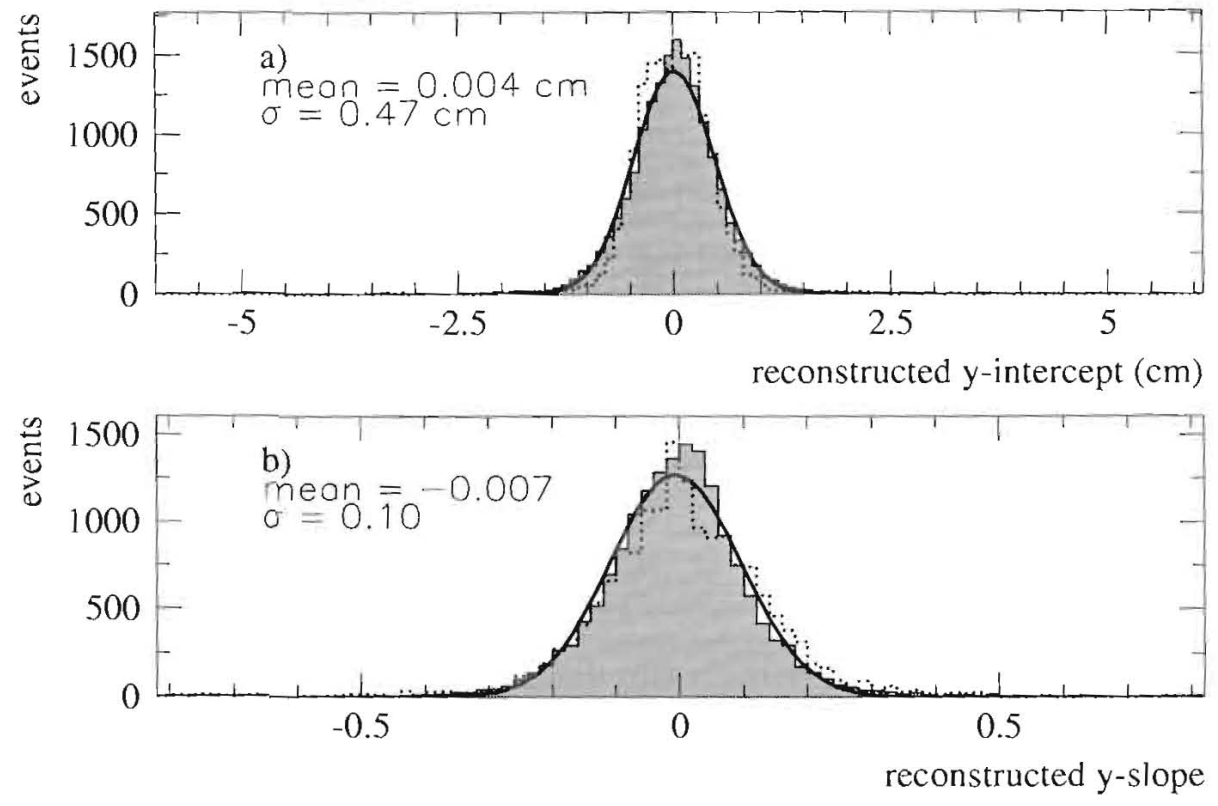

Figure 4.9: Track fitting results for (a) y-intercept and (b) y-slope from test session I data with the beam centered on the detector. The mean and sigma values from a Gaussian fit to each distribution are indicated. The resulting position and angular resolutions are $0.44 \mathrm{~cm}$ and $5.6^{\circ}$ respectively. The dotted lines show results from fits to the simulation data of this test configuration. 

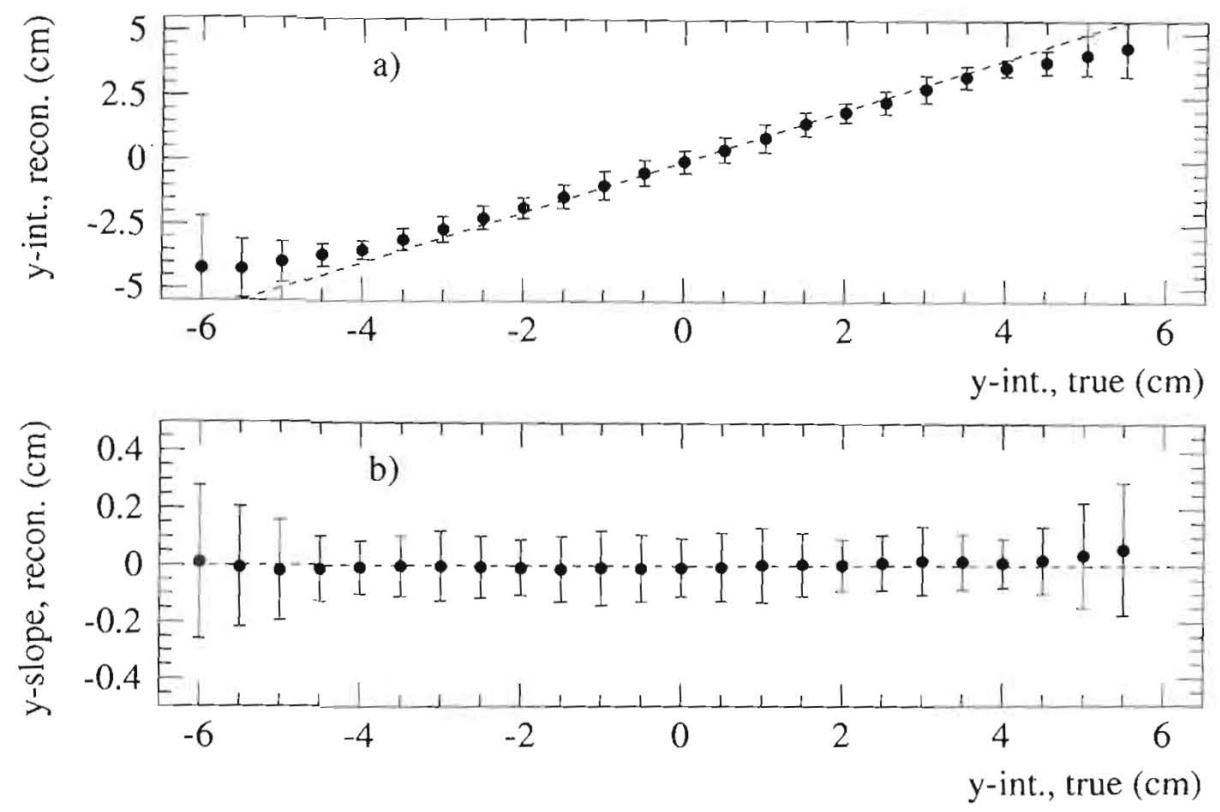

Figure 4.10: Track fitting results for (a) y-intercept and (b) y-slope from test session I vertical beam scan data. The error bars indicate the resolution, corrected for the size of the trigger scintillators. The dotted lines indicate the true values for the reconstructed quantities

and $y$-slope are reconstructed for a large range in $y$-position of the proton beam. The reconstructed $y$-intercept values deviate from the true values beginning at approximately $\pm 4 \mathrm{~cm}$ because of light loss near the detector edges (the fibers are located at $y=-4,-2,0,+2,+4 \mathrm{~cm})$.

Data was collected in test session I with the detector rotated about the horizontal $\mathrm{x}$-axis by $\pm 10^{\circ}$ (see Fig. 4.4). This tests if the track fitting procedure is robust enough to correctly reconstruct tracks for events where the track is at different distances from all the fibers in a particular row. The results from this exercise, shown in Figure 4.11, yield values for $y$-intercept and y-slope that are symmetric between $\pm 10^{\circ}$ and the widths are about the same as for $0^{\circ}$ tracks shown in Fig. 4.9. The means are consistent with the expected geometry except for a slight bias toward smaller absolute slopes. The expected value for the $y$-slope is $\pm 10^{\circ}$. The peaks of the distributions are reasonably consistent with that, however, the fit Gaussian is shifted slightly to lower absolute values.

In test session II, two different fiber types were tested in the same apparatus, 

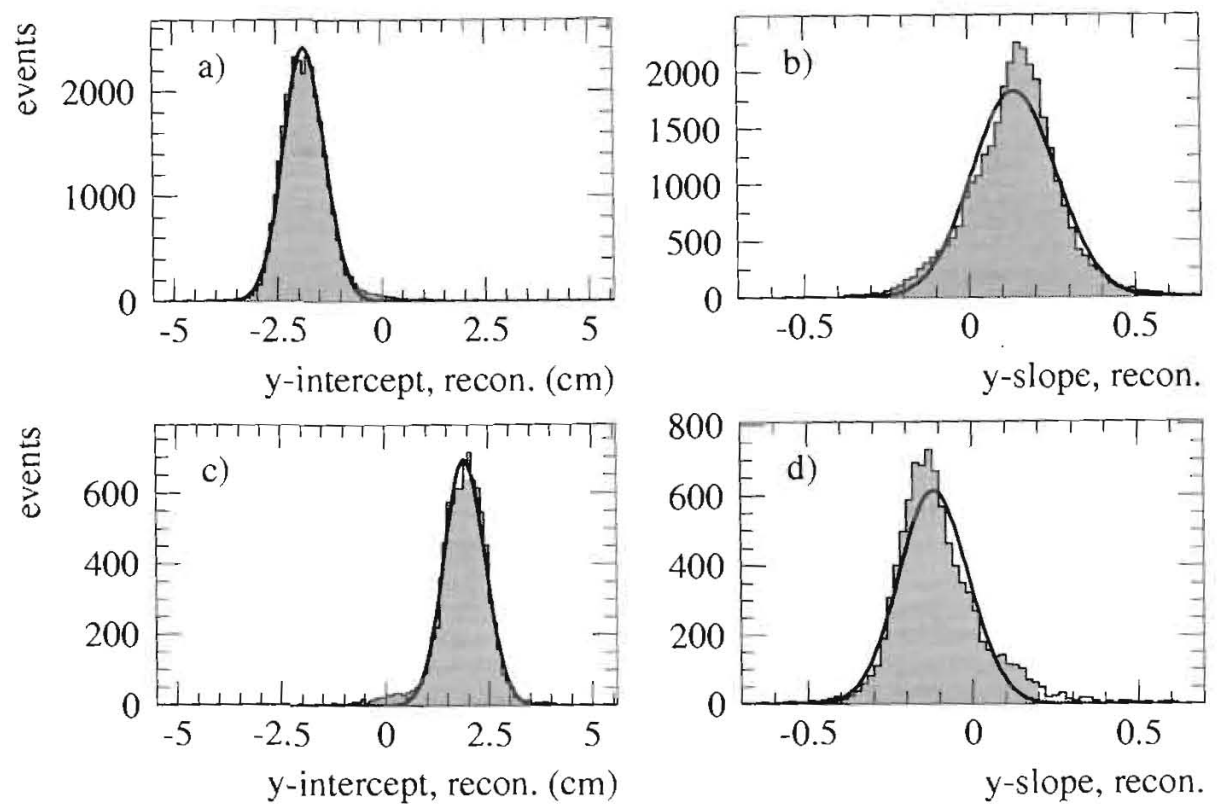

Figure 4.11: Track fitting results to test session I runs with beam at $\pm 10^{\circ}$. Distributions are shown for (a) y-intercept and (b) y-slope with a beam angle of $+10^{\circ}$ and (c) $y$-intercept and (d) y-slope with a beam angle of $-10^{\circ}$.

so tracking was not possible with this configuration. However, to determine if the better localization observed in test session II would lead to better tracking resolution, the simulated data were subjected to the track fitting procedure. The simulation reproduces the track fitting results well for test session I data as can be seen in Figure 4.9 where the results from the simulation are plotted together with the test data. For test session II simulated data, the same form of the light amplitude relation (Eq. 4.2) was used but with parameters, $L_{0}=18.1$ photoelectrons and $s=1.81 \mathrm{~cm}$. This agrees with the measured function.

The track fitting results from simulated test session I and II data are shown in Figure 4.12. The test session I simulation results (Figs. 4.12a,b) can be compared to the distributions from the data shown in Fig. 4.9. The agreement is good. As can be seen by comparing the simulation results of test session I and II, the more rapid decrease of the light amplitude with distance is predicted to result in significantly better reconstruction. A comparison of the widths for both intercept and slope between the two simulations shows that the resolution is improved by a factor of approximately two. From these results, the combination of short attenuation length scintillator and 

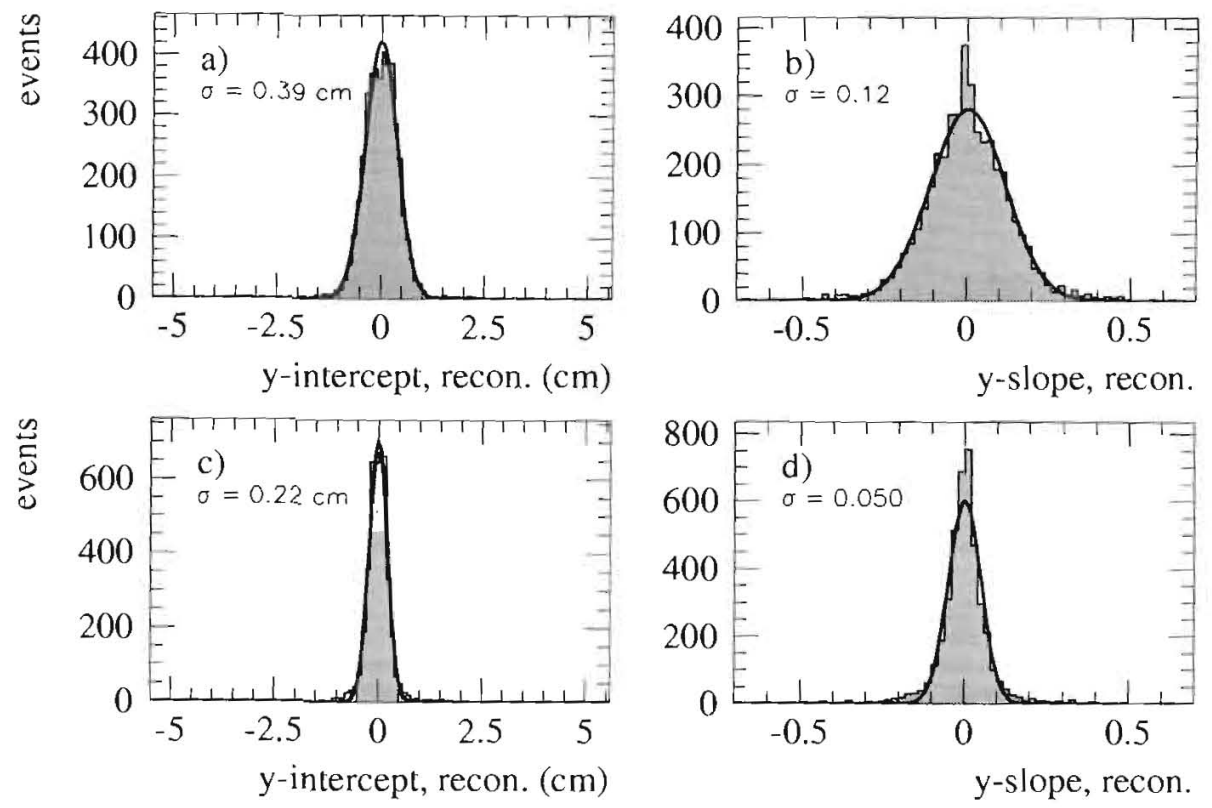

Figure 4.12: Track fitting results to simulated data. Distributions are shown for (a) $\mathrm{y}$-intercept and (b) y-slope from test session I simulated data and for (c) y-intercept and (d) y-slope from test session II simulated data. The curves show Gaussian fits to the data.

UB fibers (BCF-99(UB) from Table 4.2) maximize tracking capabilities crucial for FINeSSE, and therefore will be employed in the FINeSSE detector.

\subsubsection{Neutron Tagging}

In addition to the excellent tracking "scibath" achieves, the light output and collection, even at low energies, in the Vertex Detector, allow for neutron tagging, necessary in reducing error on the FINeSSE $\Delta s$ measurement.

Neutrons produced in neutrino interactions are important to tag for the $\Delta s$ analysis in order to differentiate $\nu p \rightarrow \nu p$ events from $\nu n \rightarrow \nu n$ events. A delayed ( 200 $\mu \mathrm{s})$ neutron capture signal from the nuclear-capture reaction $n+p \rightarrow d+\gamma(2.2 \mathrm{MeV})$ correlated with a beam trigger, indicates a $\nu n \rightarrow \nu n$ event. Chapter 5 discusses efficiencies for detecting these interactions. Here, the detector response to the capture signal is described.

The $2.2 \mathrm{MeV} \gamma$ tag from neutron capture produces approximately 50 photons 


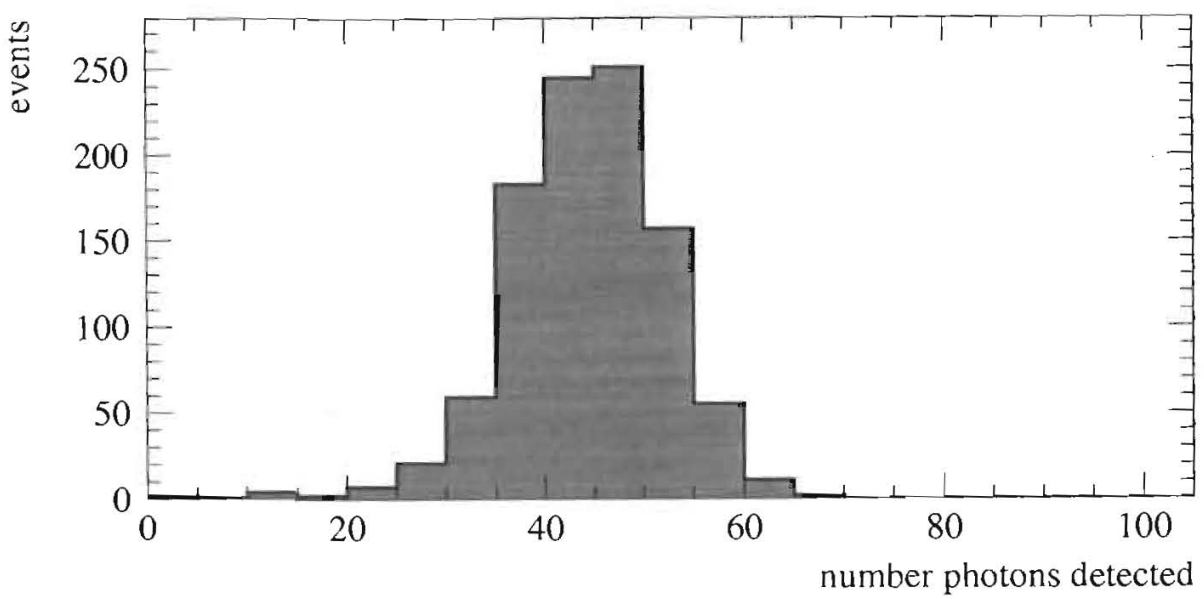

Figure 4.13: GEANT3 simulation of number of detected photons for $2.2 \mathrm{MeV} \gamma \mathrm{s}$ in the FINeSSE detector. This clear signal allows for neutron tagging, crucial for the $\Delta s$ analysis.

read off by 50 hit fibers (about one photon read off per fiber). Figure 4.13 shows a Monte Carlo simulation of detector response to these low energy $\gamma \mathrm{s}$. The light sharing over a number of localized fibers make this a clear signal for neutron capture.

\subsection{The Muon Rangestack}

The Muon Rangestack is located just downstream of the Vertex Detector. This subdetector, in conjunction with the Vertex Detector, is designed to range out muons with energies up to $1.5 \mathrm{GeV}$, allowing for a measurement of the muon energy. These characteristics are needed to enable the reconstruction of $\mathrm{CC} \nu_{\mu}$ events. Because the muons from high energy $\mathrm{CC} \nu_{\mu}$ events tend to be produced at forward angles, the Rangestack need only provide adequate acceptance for coverage in the forward direction.

The iron absorber planes and tracking granularity in the Rangestack are designed to meet these requirements. The stack is comprised of $4 \times 4 \mathrm{~m}^{2}$ alternating planes of scintillator strips and iron absorber with an overall depth of $0.85 \mathrm{~m}$ (0.98 m including support structure) in the beam direction, and a weight of 100 tons. Of the $0.85 \mathrm{~m}$ thickness, $0.24 \mathrm{~m}$ is scintillator and $0.61 \mathrm{~m}$ is iron. This design meets the physics requirements while minimizing cost and space demands. 


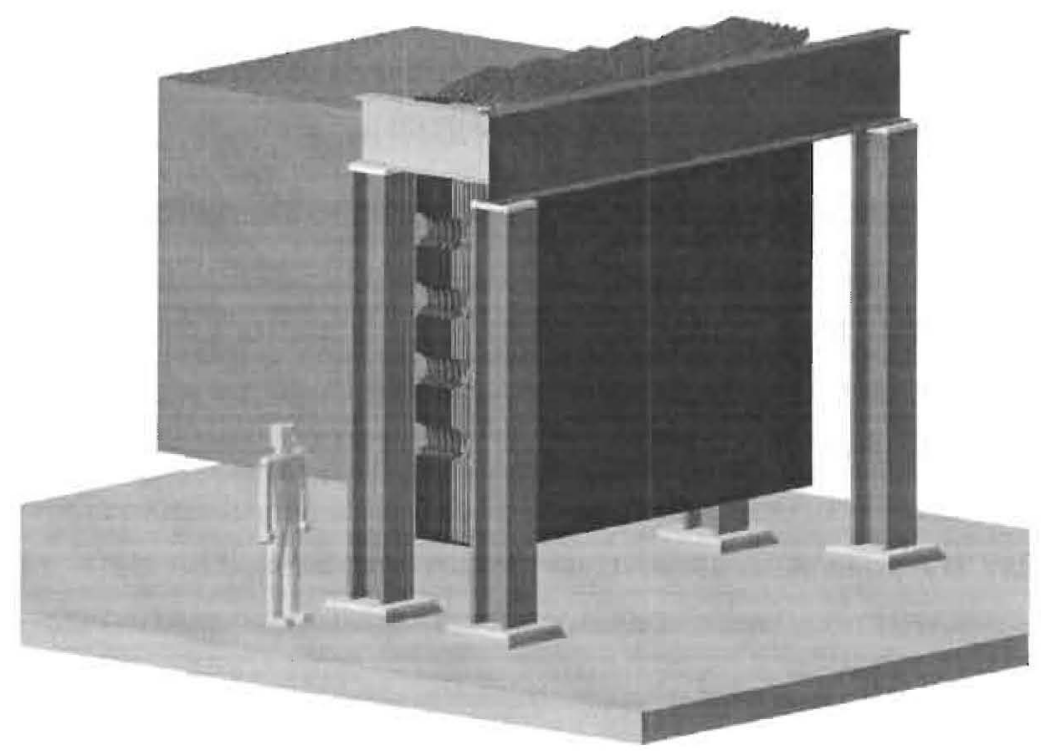

Figure 4.14: An schematic drawing of the detector from the backside showing the multiple layers of steel and scintillator that comprise the Muon Rangestack.

The Rangestack may be seen in Figure 4.14 situated behind the Vertex Detector. The structural steel shown supporting the stack is a conceptual design. The PMT enclosures or clear fiber bundles surrounding the Rangestack are not shown here. Details on the design of the Rangestack can be found in Reference [56]

\subsection{Signal Readout: Phototubes and Electronics}

A common design for signal readout will be used for the Vertex Detector and the Muon Rangestack. Requirements for the readout system include independent amplitude and timing measurements for each channel. The amplitude information is used in the track reconstruction and for $d E / d x$ measurement for the particle tracks; timing information is used to assemble the hits (rejecting spurious noise hits and background tracks), to correlate with the beam spills, and in the analysis of secondary events such as muon decay and nuclear decay in the active volume following the capture of a neutron produced in the primary event. In the Vertex Detector, the full scale signal range will extend to $>50 \mathrm{PE}$, with an amplitude resolution of $<0.1 \mathrm{PE}$. The timing resolution 
is not crucial, but needs to be $\sim 10$ ns to reduce spurious hits and background tracks. We have made preliminary tests, both with the scibath prototype mentioned above and with a test pulser, and simulations, demonstrating this level of performance using using a low-cost 10-bit A/D converter at a $10-20 \mathrm{MHz}$ sample rate.

The readout must be sensitive both to the primary interaction events (which are in time with the beam spill) and to secondary events as described above. It is also useful that it be sensitive to cosmic ray muon tracks, for calibration purposes. For these reasons, the front end electronics must be self-triggering and have a relatively low dead-time. Dead-time of the proposed system is about $1 \mu \mathrm{s}$, and it applies independently to each channel - it is a matter of recovery of the front end electronics only, there is zero dead-time associated with the data readout. A external global trigger will also be implemented, which can be used to force an event readout and/or a charge pulser event, for diagnostic purposes.

The readout system being designed to meet these requirements combines a Hamamatsu R7600-00-M64 64-channel multi-anode photomultiplier tube (MAPMT) and custom readout electronics in a 64-channel "readout module." It integrates all the necessary front end electronics, HV bias circuits, charge-injection test pulsers, and data acquisition and control communications in a compact and rugged module. This design is quite similar to that recently developed for the STAR Endcap Electromagnetic Calorimeter shower-maximum detector [57]. The module is a sealed, conductively cooled unit suitable for submerged operation inside the oil tank (for the Vertex Detector application). In the Muon Rangestack the readout modules will be mounted on the sides of the detector. We are also exploring an option to mount the Vertex Detector readout outside the oil tank, in which case it would consist of air-cooled $3 \mathrm{U}$ form-factor plug-in modules. The Vertex Detector requires 300 modules for readout of the active volume and 12 modules for the veto shield. The Muon Rangestack requires an additional 34 modules.

\subsection{A Possible Future Upgrade: Liquid Argon Time Projection Chamber}

A Liquid Argon TPC is not necessary to achieve the physics goals presented in this Letter, but, if installed at some time in the future, has the potential to substantially 
enhance the experiment. Liquid Argon TPC's combine bubble chamber quality resolution with active readout giving them great promise for precision neutrino scattering measurements. However, the technology has taken some thirty years to reach maturity. While the ICARUS collaboration in Europe has shown results from prototype Liquid Argon TPCs as large as 600 tons [58], the technology has not yet been widely used outside of this collaboration.

The feasibility of this technology for the set of measurements set forth here is currently under active study. If demonstrated to be feasible and worthwhile through an on-going R\&D program and Monte Carlo studies, a small ( $\sim 20$ ton) Liquid Argon TPC could be installed upstream of the existing Vertex Detector to enhance these precision neutrino scattering measurements. The rest of this section provides a brief description of how these detectors work and some of the issues regarding construction and operation of a $\sim 20$ ton Liquid Argon TPC detector.

\subsubsection{Detection Technique and Detector Construction}

Charged particles are detected in Liquid Argon TPCs via ionization electrons produced along the path of the passing particle. These ionization electrons drift over several meters to the edge of the chamber where they are readout via wire chamber planes. In order for the drifting electrons to travel on the order of a meter in Argon, without being captured, the impurity levels must be less than $0.1 \mathrm{ppb}$ in the Argon. This purity level is achieved by passing the Argon through oxisorb/hydrosorb standard filters. The entire detector must be housed within a large cryostat and have an active Argon purification system.

Ionization electrons are read out on three wire chamber planes with wires at 3 $\mathrm{mm}$ pitch, rotated by $60^{\circ}$ with respect to each other. The first two planes read the charge induced by the passing electrons and the third collects the charge.

Scintillation light is also produced in copious amounts in Liquid Argon. ICARUS has detected scintillation light levels in its 600 ton prototype via Hamamatsu 8 inch Photomultiplier tubes [58]. Scintillation light may provide an additional handle for particle identification.

In a neutrino beam, the time of an event can typically be determined to $\sim 1 \mu \mathrm{s}$ from beam timing and scintillation light signature. This corresponds to a negligible $1 \mathrm{~mm}$ drift in the Liquid Argon. This information combined with the drift time, and 
the wire chamber readout, allows for a 3D track reconstruction. Figure 4.15 shows a stopped cosmic ray muon track and the decay electron as read out from the 600 ton ICARUS Liquid Argon TPC [58].

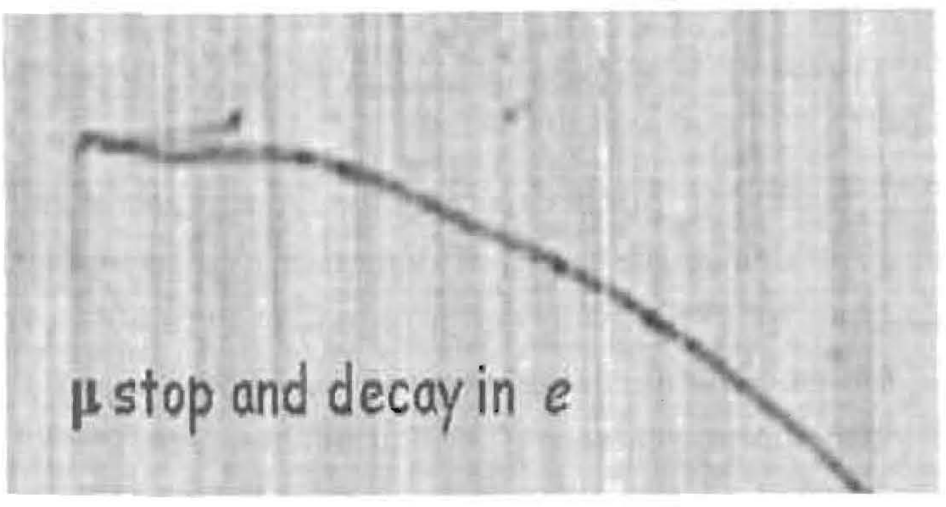

Figure 4.15: A stopped cosmic ray muon and its decay electron, in the ICARUS T600 prototype [58].

\subsubsection{R\&D program}

In order to independently understand and verify the feasibility of this technology, we are building a small, $\sim 50$ liter Liquid Argon TPC prototype. The goal of this work is to demonstrate the technology within our group and to allow us to identify any unforeseen problems. A rendering of this prototype is shown in Figure 4.16. In conjunction with building a prototype, we are studying the specifics of neutrino interactions in this kind of detector using detailed Monte Carlo simulations. Throughout this process, we have been in contact with members of the ICARUS collaboration who have been extremely helpful and supportive of our initiative [59]. 


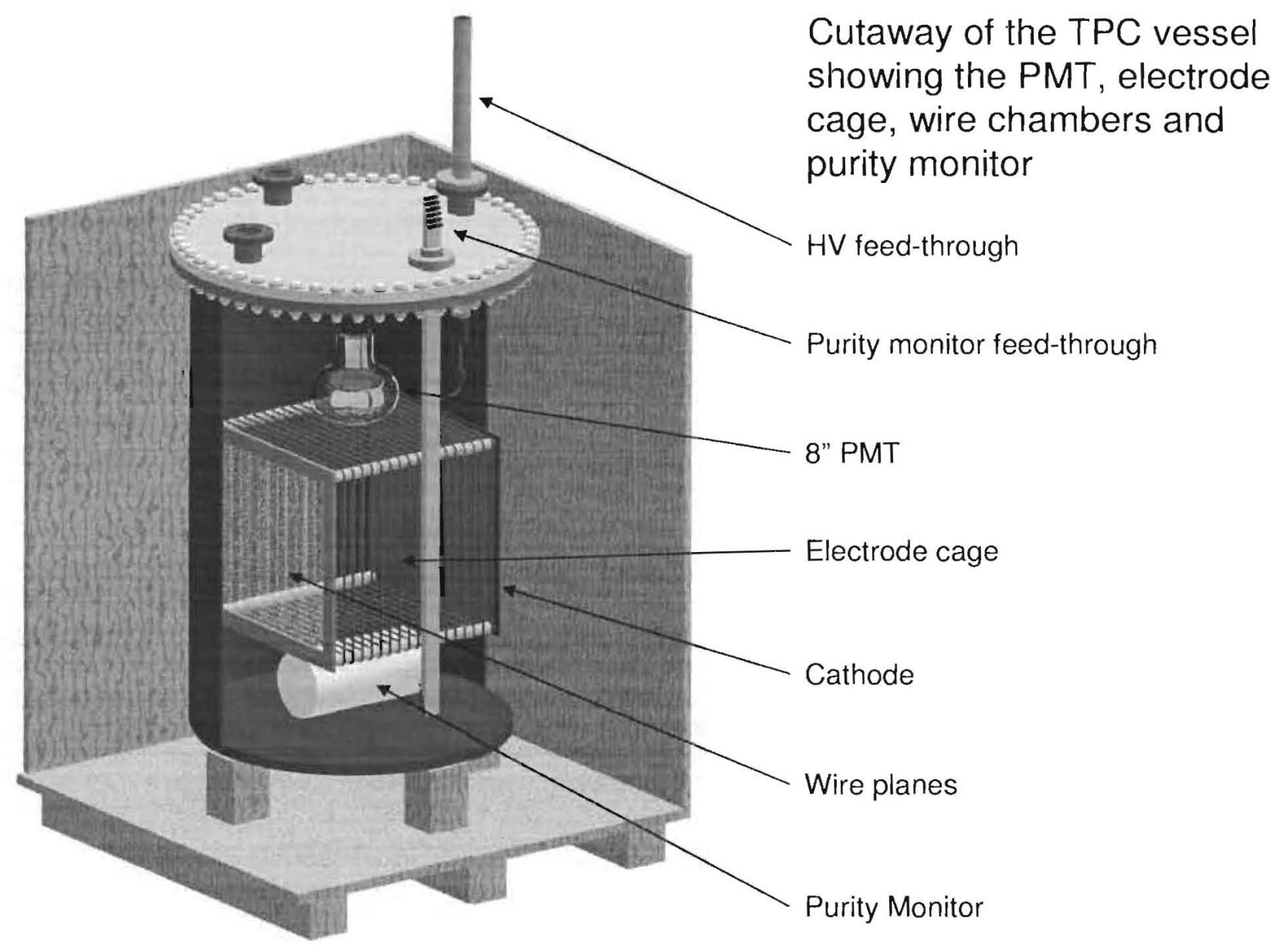

Figure 4.16: Rendering of a small Liquid Argon TPC prototype designed to demonstrate the technology as well as study low energy charge and light production in Argon. Prototype work is underway at Yale University. Design work done by Bartoszek Engineering. 


\section{Chapter 5}

\section{Physics Sensitivity}

Physics events are simulated using a full GEANT3 Monte Carlo. Reconstruction of these events demonstrates that the physics goals of this experiment can be realized.

\subsection{Introduction to Interactions in the Detector}

The event rates expected in the Vertex Detector are listed in Tables 3.1 and 3.2 for neutrinos and anti-neutrinos respectively. The most important and most prevalent events occurring in the detector are listed in Table 5.1, along with a description of how they are observed in the detector.

\begin{tabular}{llcl}
\hline event name & reaction & \# tracks & description \\
\hline $\mathrm{CCQE}$ & $\nu n \rightarrow \mu^{-} p$ & 2 & $\mu, \mathrm{p}$ : two-body kinematics \\
$\mathrm{NCp}$ & $\nu p \rightarrow \nu p$ & 1 & $\mathrm{p}$ : two-body kinematics \\
$\mathrm{NCn}$ & $\nu n \rightarrow \nu n$ & 0 & $\mathrm{n}$ : extraneous visible tracks from $\nu p$ \\
& & & scattering, delayed n-capture pho- \\
& & & ton \\
$\mathrm{CC} \pi$ & $\nu n \rightarrow \mu^{-} X \pi^{ \pm, 0}$ & $>\approx 2$ & Not two-body kinematics \\
$\mathrm{NC} \pi$ & $\nu p, n \rightarrow p, n X \pi^{ \pm, 0}$ & $>\approx 1$ & Not two-body kinematics \\
\hline
\end{tabular}

Table 5.1: Summary and description of event types in the detector. "\# tracks" means typical number of charged particle tracks of significant energy. 


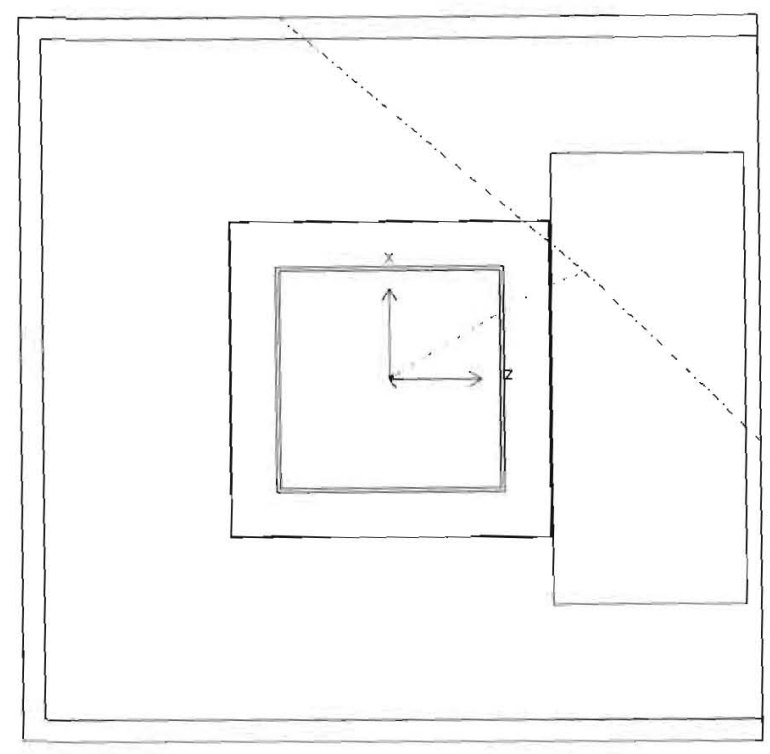

Figure 5.1: A typical $\nu n \rightarrow \mu^{-} p$ reaction in the detector. The event vertex is at the origin of the indicated coordinate system. In this example, the $\mu^{-}$leaves the Vertex Detector, stops in the range stack, and decays. The two neutrinos (dot-dashed lines) from the muon decay are seen exiting the apparatus. The short recoil proton track is visible as a short stub at the origin.

Examples of typical simulated events are shown in Figures 5.1-5.3. In these figures, charged hadrons are shown as solid lines, muons as wide dashed lines, neutrinos and neutrons as dot-dashed lines, and photons as dotted lines. The detector is shown from the side.

The individual particles within a particular event will be identified via their track length, energy loss density, " $d E / d x$ ", and their decay pattern:

- $\mu^{ \pm}$: Long tracks with low $d E / d x$. High-energy $\mu^{ \pm}$will exit the Vertex Detector, enter the veto, and perhaps the range stack. For $\mu^{ \pm}$that stop in an active area, the decay (Michel) electron will be observed.

- $p$ : Short tracks with high $d E / d x$. A $100 \mathrm{MeV}$ proton travels approximately $10 \mathrm{~cm}$ in liquid scintillator.

- $n$ : Extraneous tracks from $\nu p$ scattering. Occasionally transfer enough energy in one collision so as to be misidentified as a $p$ track. Will thermalize and capture in the detector yielding a delayed $2.2 \mathrm{MeV} \gamma$. 


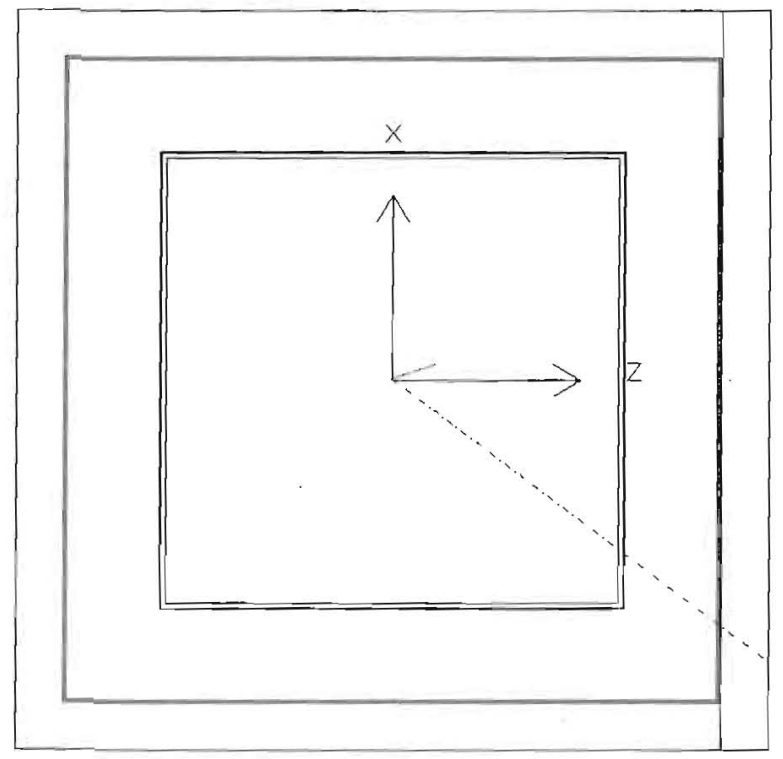

Figure 5.2: A typical $\nu p \rightarrow \nu p$ reaction expected in the detector, generated at the origin. The short proton track (solid line) can be seen, as can the final state neutrino (dot-dashed line) which exits the apparatus. In this view, only the Vertex Detector is shown.

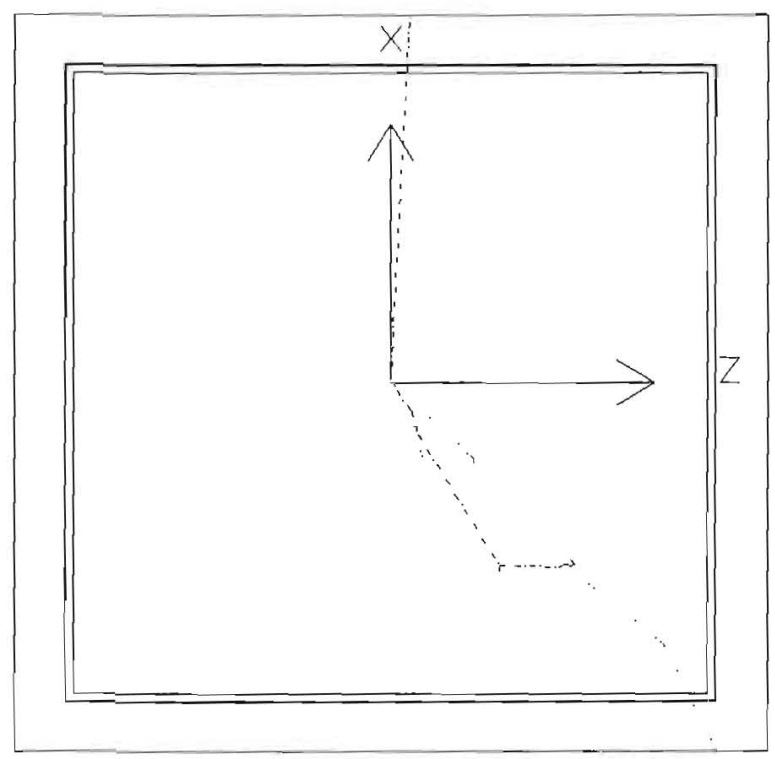

Figure 5.3: A typical $\nu n \rightarrow \nu n$ reaction expected in the detector. Several interactions of the final state neutron can be seen. The final state neutrino (dot-dashed line) exits the apparatus. In this view, only the Vertex Detector is shown. 


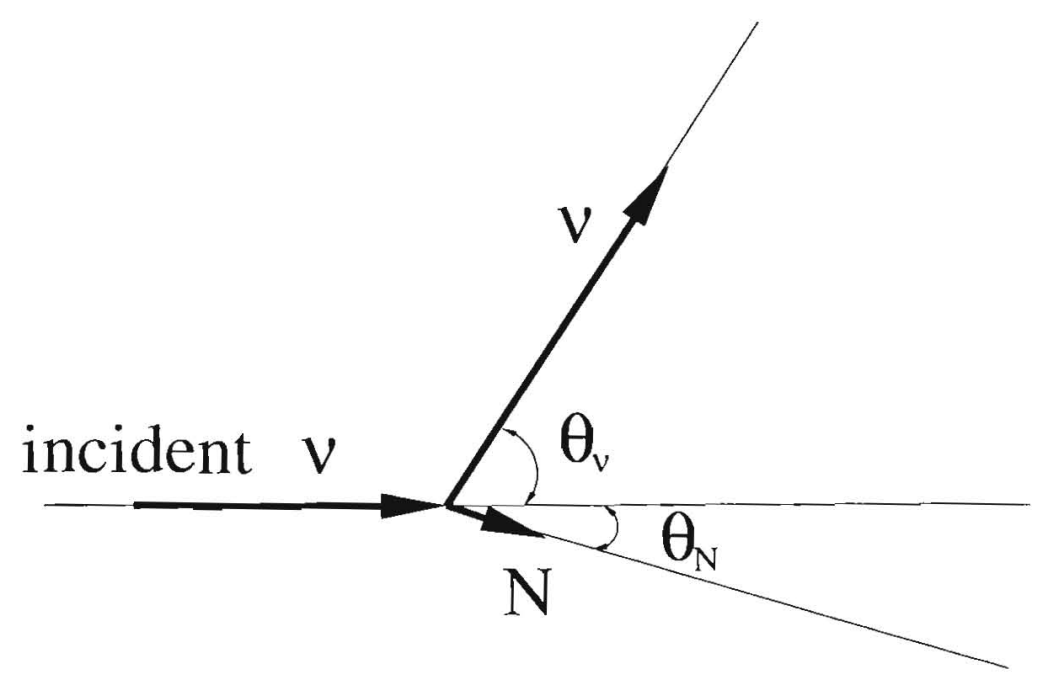

Figure 5.4: Illustration of the scattering angles for the NC elastic $(\nu p \rightarrow \nu N)$ reaction. The CCQE $\left(\nu n \rightarrow \mu^{-} p\right)$ reaction is similar, with the $\mu^{-}$in place of the $\nu$.

- $\pi^{ \pm}$: Longish tracks that look like $\mu^{ \pm}$. For $\pi^{ \pm}$that decay in the active area of the detector, the subsequent $\mu^{ \pm}$and $e^{ \pm}$can be observed.

- $\pi^{0}: 2$ hit clusters from the $\pi^{0}$-decay $\gamma$ showers.

- $e^{ \pm}: 1$ "fat" track from the $e^{ \pm}$shower.

\section{Event Kinematics}

The two-body kinematics of NC elastic $(\nu N \rightarrow \nu N)$ and CCQE $\left(\nu n \rightarrow \mu^{-} p\right)$ interactions may be better understood by examining the scattering angle definitions in Figure 5.4 and the kinematic ellipses in Figure 5.5.

Due to the energy of the incident neutrino and the low mass of the muon compared to the hadron mass, the kinematics of the NCp and CCQE events are almost identical above $E_{\nu} \approx 300 \mathrm{MeV}$. This simplifies the analysis and interpretation of the $\mathrm{NC}$ and CCQE event reconstruction. As can be seen in Figure 5.5, the final-state lepton may have any angle; the outgoing proton has a maximum lab angle of $90^{\circ}$. The correlations can also be seen in this figure. An event with a lower-energy high-angle lepton is paired with a low-angle high-energy proton (as in Fig. 5.4). And, a low-angle high-energy lepton is accompanied by a high-angle low-energy proton. 


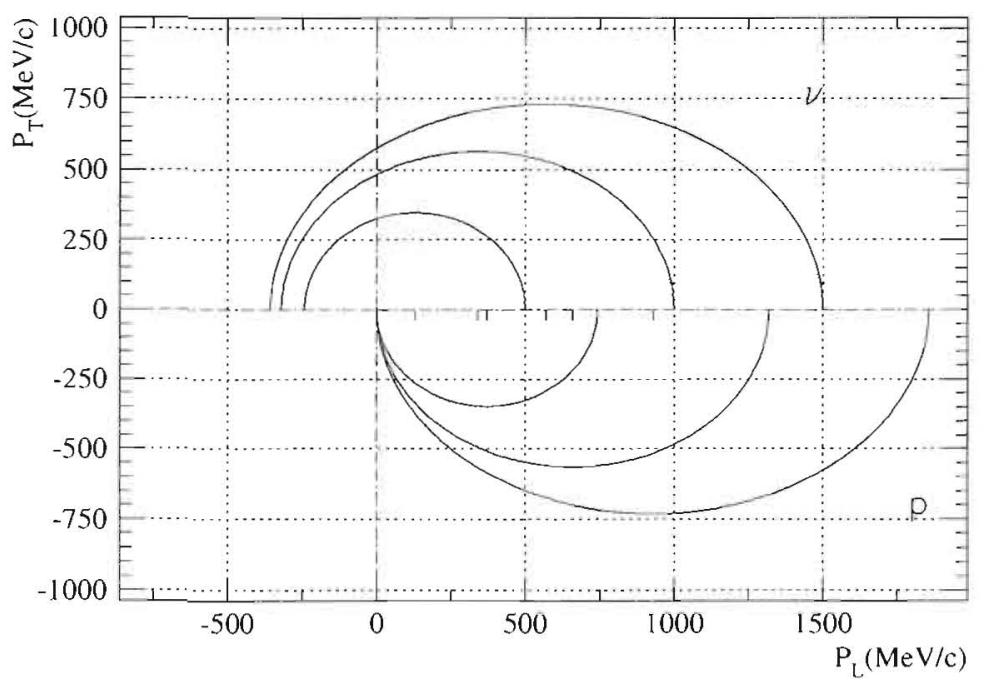

Figure 5.5: Kinematic ellipses for the NC elastic $(\nu p \rightarrow \nu p)$ reaction. These are also valid for the CCQE $\left(\nu n \rightarrow \mu^{-} p\right)$ reaction. The ellipses of increasing size indicate the lab momentum for the event at $E_{\nu}=500,1000,1500 \mathrm{MeV}$. The longitudinal component (parallel to the beam) of the particle momentum is plotted on the $\mathrm{x}$ axis and the transverse component on the y-axis. In a particular event, the particle momentum vector is constrained to lie on the appropriate ellipse and in such a way to conserve momentum. 
Plotting energy vs. angle (Fig. 5.6) reveals strong correlations between the two variables in such two-body reactions. The events of most interest for the $\Delta s$ analysis are low $Q^{2}$ events, where the proton has a low energy and a high angle. The lepton in these events will be in the forward direction at high energy. Many of the lower energy muons range out in the Vertex Detector and veto; the most energetic ones, at small angles, enter and range out in the Muon Rangestack.

The effects of the Fermi momentum of nucleons can be seen in Fig. 5.6, which compares the final-state protons produced in CCQE scattering from nucleons bound in carbon (Fig. 5.6b) to those from free nucleon scattering (Fig. 5.6c). Fermi momentum widens the angular distribution of the outgoing proton and suppresses the number of nucleons at low momentum ("Pauli blocking"). The effect of this additional Fermi energy $(\approx 25 \mathrm{MeV}$ ) on the energy of the outgoing proton is small and will have minimal impact on measuring the $Q^{2}$ of the reaction (via $Q^{2}=2 m_{p} T_{p}$ where $m_{p}$ and $T_{p}$ are the mass and kinetic energy of the proton respectively).

The correlations shown in Fig. 5.6 will be used to reduce backgrounds from NC and $\mathrm{CC}$ single pion reactions by requiring that the reconstructed tracks obey the illustrated kinematic constraints.

\subsection{Physics Simulations}

The baseline detector, including the Vertex Detector and Muon Rangestack, has been simulated using the the GEANT3 [53] simulation package. Physics events were transported through this code, and the resulting simulated data reconstructed.

\subsubsection{Simulation of the Detector}

A diagram of the apparatus as modeled by the GEANT-based Monte Carlo program is shown in Fig. 5.7. The Vertex Detector is simulated as wavelength-shifting (WLS) fibers immersed in liquid scintillator with the same geometry as described in Section 4.2. The fiber support structure and tank walls are also included in the simulation. The area outside of the fiber area in the scintillator tank (the "veto") is active in the simulation. The Muon Rangestack, downstrean of the Vertex Detector, is implemented with the geometry described in Section 4.3, which consists of alternating planes of plastic scintillator and iron. 

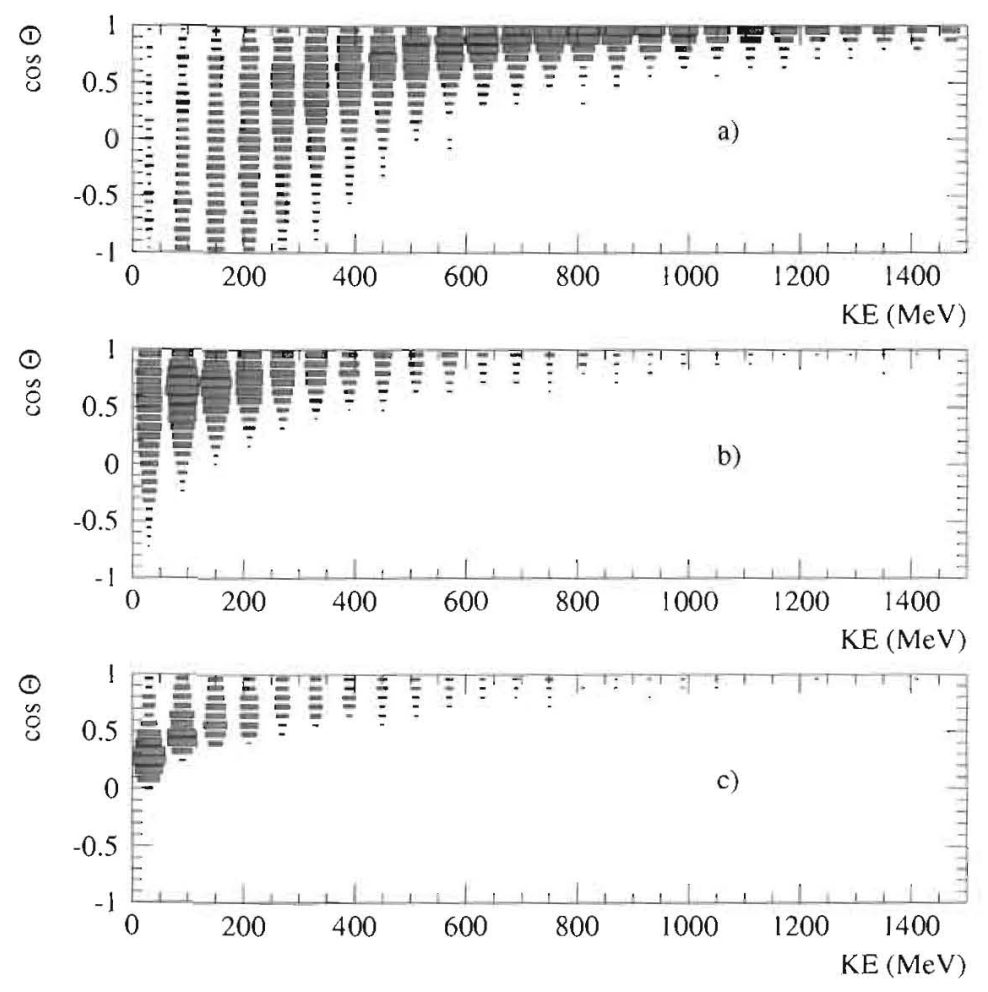

Figure 5.6: $\cos \theta$ vs. kinetic energy for the a) $\mu$, b) proton in CCQE scattering from bound nucleons, and c) proton in CCQE scattering from free nucleons. Protons in $\mathrm{NCp}$ reactions will show the same distribution as those in b).

In the inner region of the Vertex Detector, scintillation light and Čerenkov radiation from passing charged particles are simulated. Photons thus produced are tracked until they impinge upon a WLS fiber or the edge of the detector volume, or are absorbed. In the outer $50 \mathrm{~cm}$ (veto) region of the liquid scintillator and in the Muon Rangestack, individual photons are not tracked - only energy loss is recorded. This is not important in these regions as photostatistics are not crucial.

In the active region of liquid scintillator, it is estimated that 5000 scintillation photons are produced in the liquid scintillator for every $1 \mathrm{MeV}$ of energy deposited by a charged particle [54]. The absorption and capture efficiency of photons intersecting a WLS fiber is estimated to be 5\% [54]. These fibers have typical attenuation lengths of $2.5 \mathrm{~m}$, and the quantum efficiency of the PMT is approximately $20 \%$. As a result, approximately $10 \%$ of the photons emitted in the capture cone of the fiber will make it to the PMT and produce a photoelectron. Combining these two factors yields 


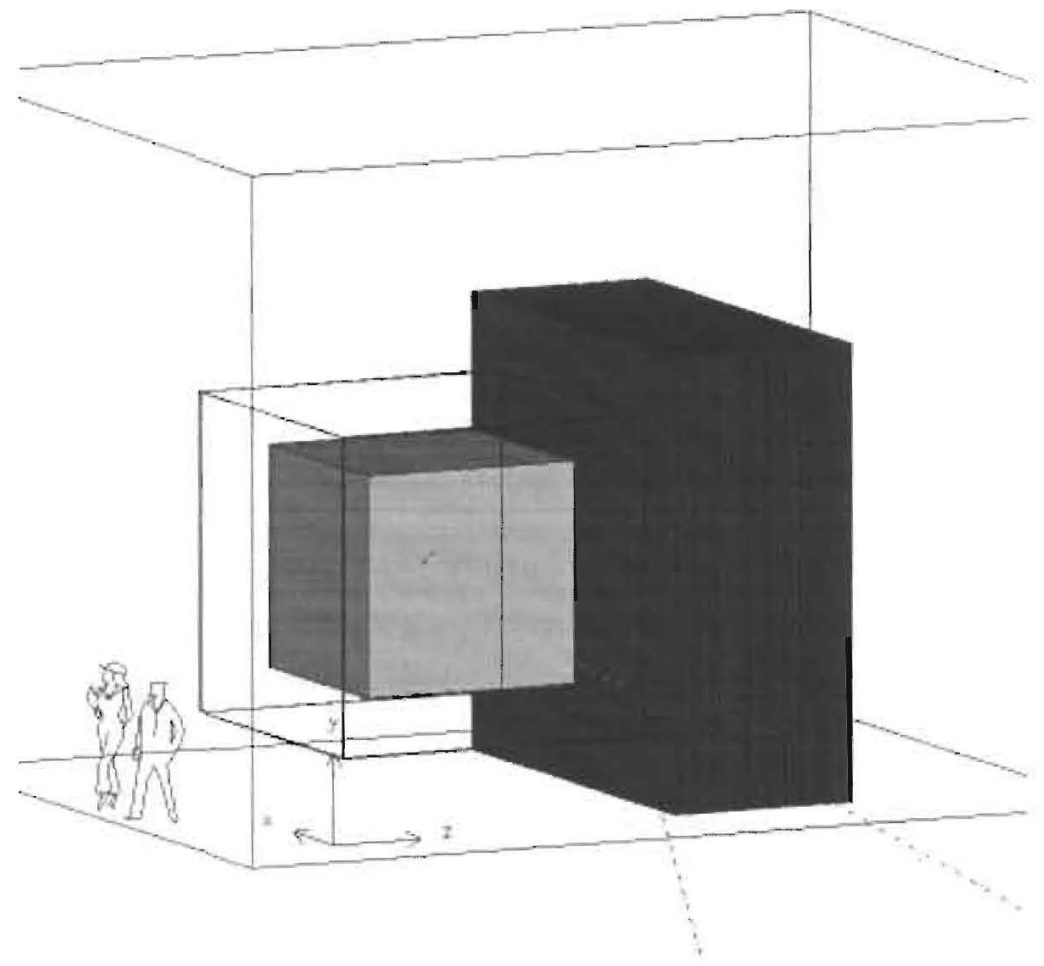

Figure 5.7: Diagram of the detector geometry as simulated by GEANT with a superimposed CCQE scattering event. 
an overall efficiency of $0.5 \%$ that an optical scintillation photon that strikes a WLS fiber will be detected at the PMT. To aid the speed of the simulation, the photon detection efficiency and production was combined so that $25(5000 \times 0.005)$ photons are produced per $\mathrm{MeV}$. An effective efficiency of $100 \%$ for the photons that hit a $1.5 \mathrm{~mm}$ WLS fiber was then assumed. In this way, the effects of photostatistics were properly simulated and the efficiency of the simulation was kept high. The attenuation length $(5 \mathrm{~m})$ [54] of the scintillator is fairly large compared to the size of the detector and is not a significant effect. The saturation due to large localized energy deposits ("Birks' Law") is also modeled. The production of Cerenkov photons is simulated but is negligible as the number of Cerenkov photons is only about $1 \%$ of that for scintillation.

Using these factors, the simulation predicts that a proton track passing near a fiber will create approximately 10 photoelectrons in the PMT. This is consistent with the earlier prototype test results reported in Chapter 4 (factoring in the difference in fiber length). Recent tests indicate that this number may be made higher with an optimal fiber/scintillator combination. Employing this method of tracking individual optical photons in the Vertex Detector assures that the photo statistics (with fluctuations) are properly simulated.

The simulation program can track single-particle events to study the detector response for each particle type. Alternatively, it can accept event descriptions as generated by the NUANCE MC program as described in Chapter 3 . The latter class are events that are used to predict physics sensitivities. The output of the detector simulation includes: a list of all the "hit" fibers in the Vertex Detector, a list of all the bars in the Muon Rangestack that recorded energy loss, and the total amount of energy and time of deposit in each area of the detector. These data are subsequently passed through the event reconstruction program.

\subsubsection{Event Reconstruction}

The Monte Carlo simulation sample with the Vertex Detector is analyzed with a reconstruction program employing the Hough Transformation Technique (HTT) [60]. The HTT transformation is a global track finding method that uses the hit fiber information from the $X Z$ and $Y Z$ orientations (The information from the $X Y$ orientation has not yet been used - - this information will improve the reconstruction from what 
is reported here). The coordinates of each fiber that record an amount of light over an adjustable threshold value are used to calculate $R$, the perpendicular distance from the track to the origin:

$$
R=X(Y) \sin \alpha+Z \cos \alpha,
$$

where $\alpha$ is a track angle. The track angle $\alpha$ is varied in a loop from $-90^{\circ}$ to $+90^{\circ}$, and the $R$ and $\alpha$ values for each hit are used to make an entry (weighted by the amount of light in the hit) in a histogram.

The task of track finding then reduces to locating "peaks" in this histogram. Locating single tracks is quite easy with this method. For events with multiple tracks, alternate methods have been developed and tuned to subtract the light from the first track before the algorithm is employed to find subsequent tracks. At present, the reconstruction program is limited to finding a maximum of two tracks in each of the two 2D-orientations $(X Z, Y Z)$.

The 2D-tracks are then combined to form 3D-tracks. The total energy and length of each track are also calculated, from which the $d E / d x$ of the track can be determined.

The simulated detector energy, angle, and position resolutions for $50-500 \mathrm{MeV}$ kinetic energy (KE) protons and muons are shown in Figure 5.8. This energy range is typical for the tracks that will be contained in the Vertex Detector for physics events. This proton kinetic energy range corresponds to $Q^{2}$ values ranging from $0.1-1.0 \mathrm{GeV}^{2}$ in NCp and CCQE reactions. A Gaussian fit to the energy and angle resolution yields $\Delta E=13(16) \mathrm{MeV}$ and $\Delta \theta=100(80) \mathrm{mrad}$ for protons (muons). The quantity $\Delta v$ plotted in Figure 5.8 is the distance from the calculated track origin from the true origin. The simulations of single particles predict a mean $\Delta v=9(10) \mathrm{cm}$ for protons (muons). The distribution is slightly wider for muons as they produce longer tracks. These results indicate detector performance that will meet the physics goals of the experiment. The effect of the detector resolution on the physics distributions will be shown in the following sections.

Several examples of the tracks obtained with the HTT reconstruction method from simulated data are shown in Figures 5.9 and 5.10. In these figures, the particle directions are indicated by the red(light) arrows. The reconstructed tracks and endpoints are indicated by blue(dark) lines and dots. As indicated, this method results in accurate reconstructed tracks for muons and protons down to kinetic energies of $100 \mathrm{MeV}$. 

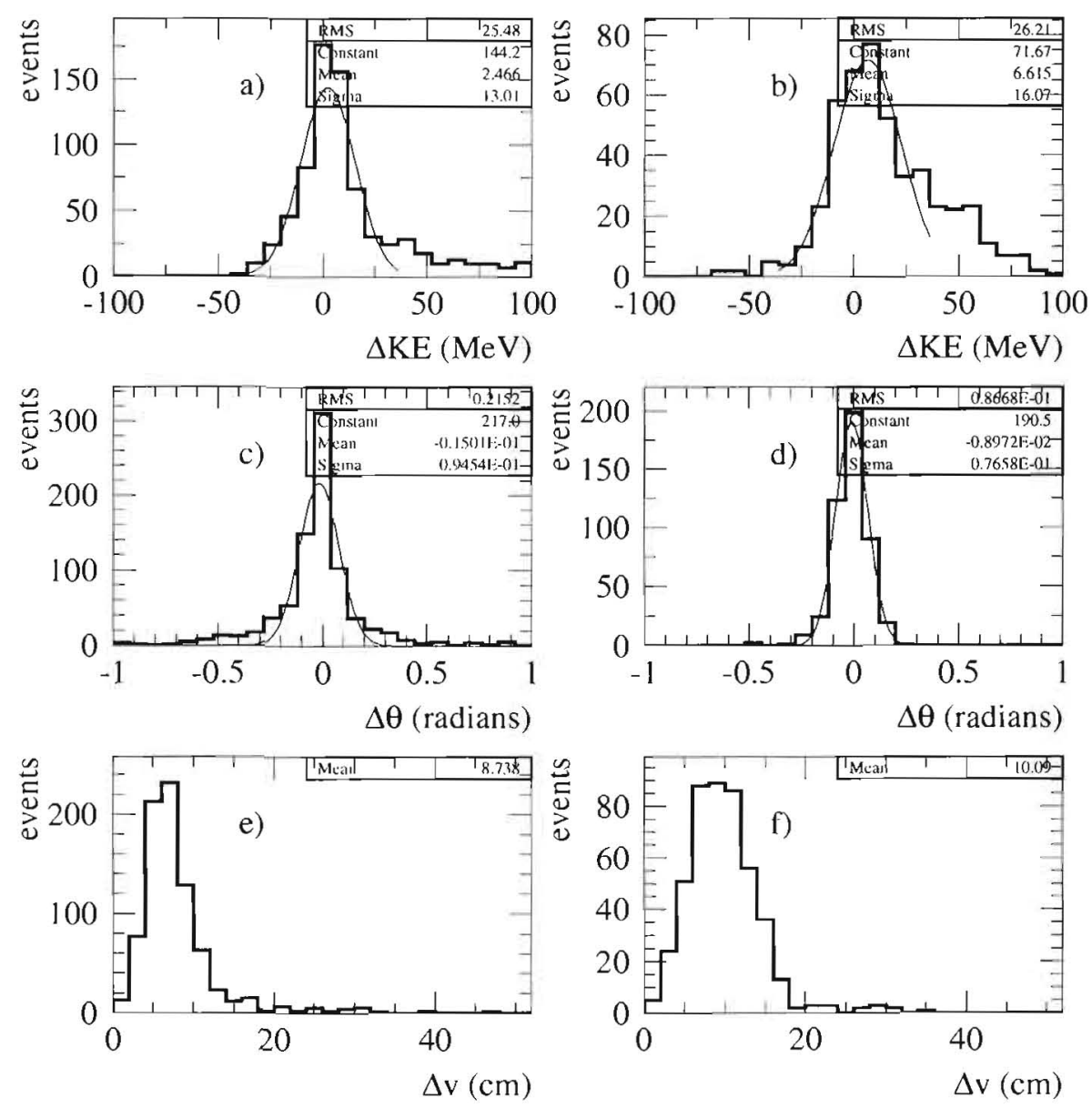

Figure 5.8: The energy $\mathrm{a}, \mathrm{b})$, angle $\mathrm{c}, \mathrm{d}$ ), and position e,f) resolution of the Vertex Detector as simulated and reconstructed for a sample of 1000 single particle events. The plots on the left a,c,e) are for $50-500 \mathrm{MeV}$ KE protons, those on the right b,d,f) for $50-500 \mathrm{MeV} \mathrm{KE}$ muons. Only tracks that were fully contained in the Vertex Detector were selected. This effectively limits the upper muon $\mathrm{KE}$ to $\approx 300 \mathrm{MeV}$. 


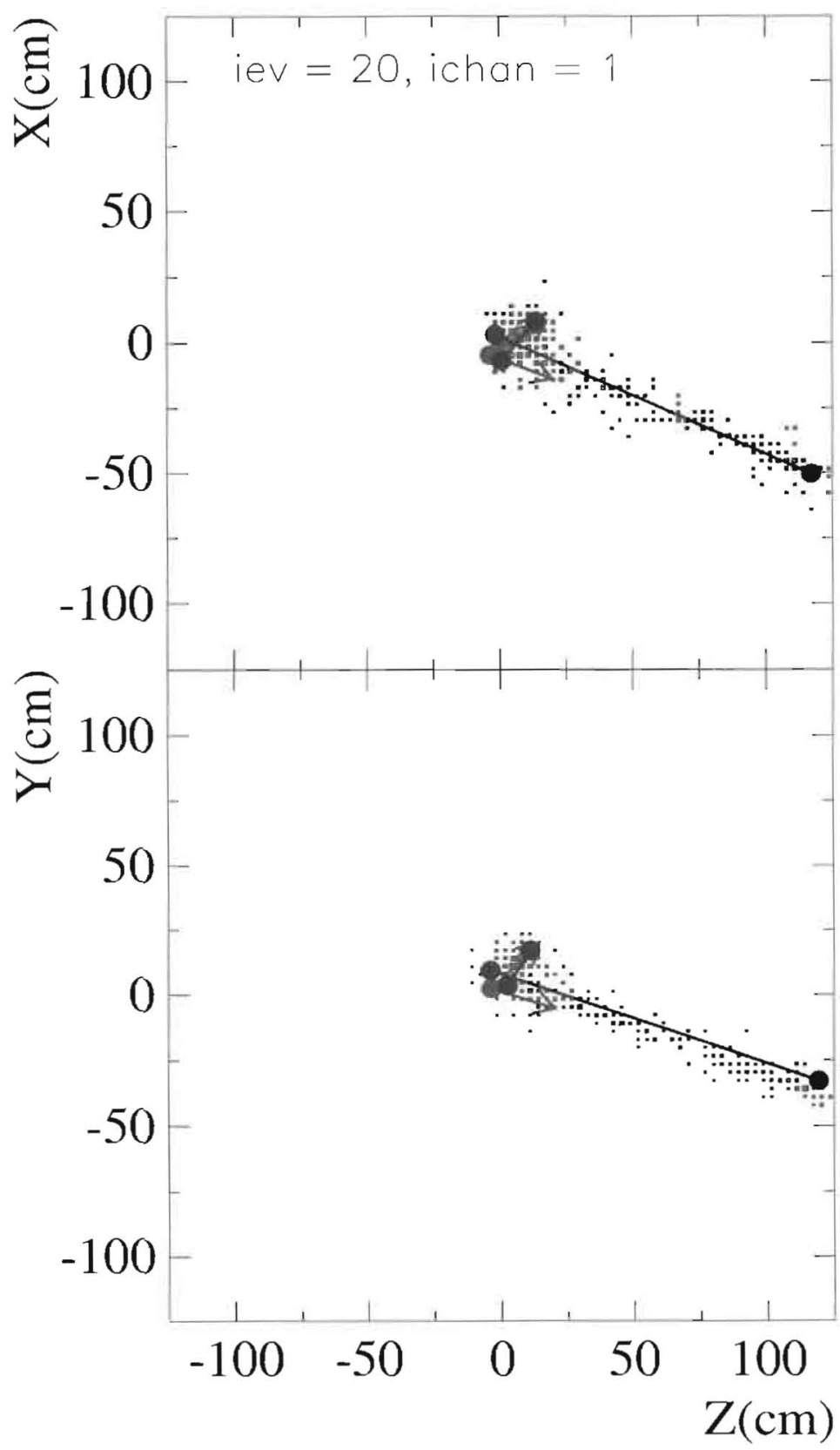

Figure 5.9: A $X Z$ (top) and $Y Z$ (bottom) projection view of a CCQE event in the simulated Vertex Detector with the reconstructed muon (long line) and proton (short line) tracks superimposed. In this event, $T_{\mu}=820 \mathrm{MeV}$ and $T_{p}=150 \mathrm{MeV}$. 


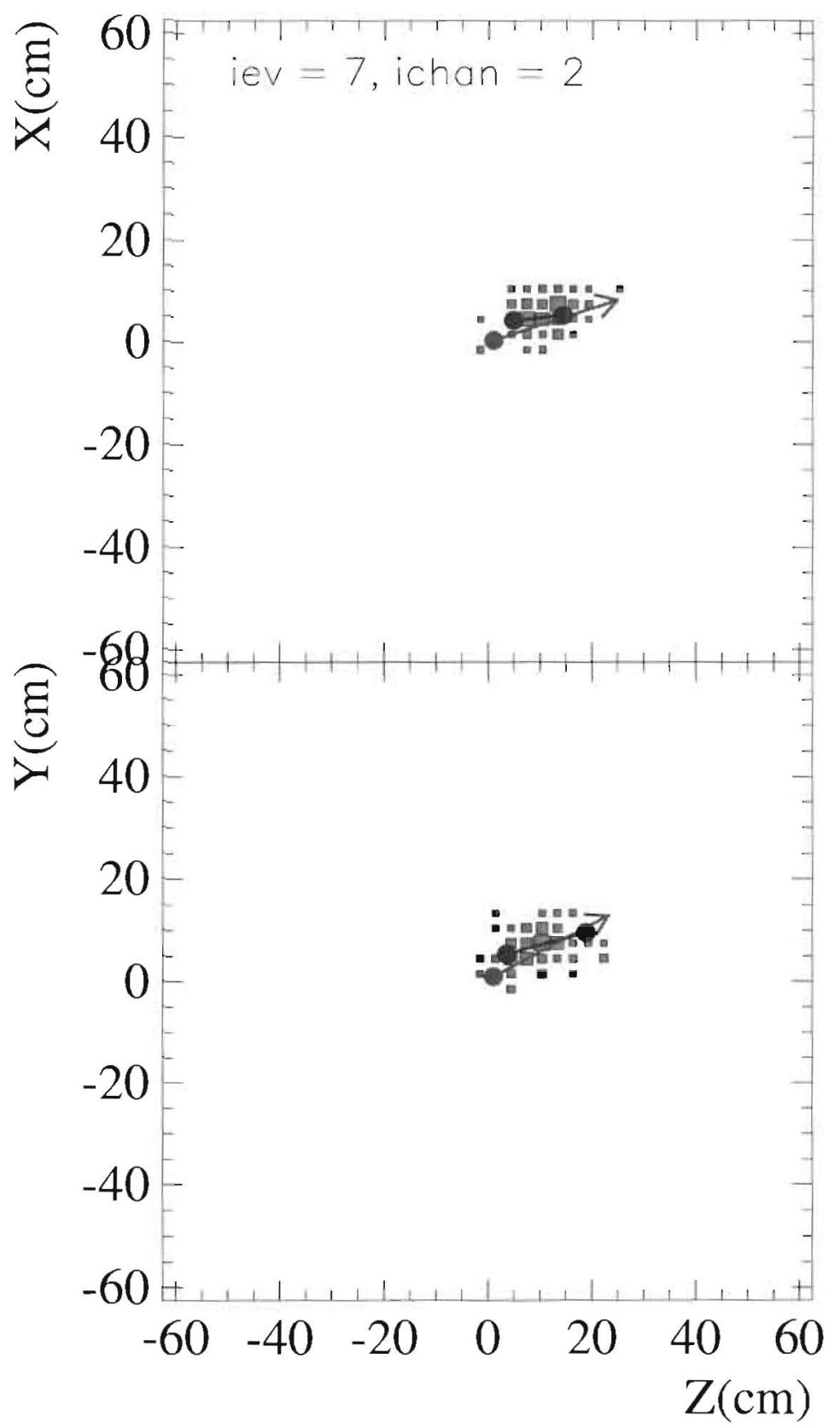

Figure 5.10: A $X Z$ (top) and $Y Z$ (bottom) projection view of a NCp event in the simulated Vertex Detector with the reconstructed proton track superimposed. In this event, $T_{p}=100 \mathrm{MeV}$. 


\subsection{Physics Sensitivities}

\subsection{1 $\Delta s$ Measurement}

As discussed in Chapter 2 , the quantity $\Delta s$ may be extracted from neutrino and anti-neutrino scattering data via a measurement of the ratio of neutral-current to charged-current scattering. A method has been developed to do this with FINeSSE with both neutrinos and anti-neutrinos. The results and estimated errors from this experiment are reported in the following sections.

\section{$\Delta s$ Measurement from Neutrino Scattering}

To extract $\Delta s$ from the neutrino data, the ratio,

$$
R_{N C / C C}=\frac{\sigma(\nu p \rightarrow \nu p)}{\sigma\left(\nu n \rightarrow \mu^{-} p\right)}
$$

will be employed. To form this ratio from the experimental data, neutral-current elastic scattering events $(\nu p \rightarrow \nu p)$ and charged-current quasi-elastic scattering events $\left(\nu n \rightarrow \mu^{-} p\right)$ will be identified, counted, and corrected for experimental efficiencies.

Both neutral-current elastic and charged-current quasi-elastic events have unique final state signatures. Neutral-current elastic scattering events ( $\mathrm{NCp}$ ) will be identified in the detector by looking for single proton tracks consistent with elastic scattering kinematics. A track is identified as a proton by a large $d E / d x$. Charged-current quasi-elastic scattering events (CCQE) are identified by looking for events with two tracks each consistent with the expected $d E / d x$. In addition, other cuts are employed to reject backgrounds. The strategy will be to maintain a compromise between large efficiency for low- $Q^{2}$ events while keeping backgrounds as low as possible. The squared four-momentum transfer, $Q^{2}$, will be determined event by event, by measuring the energy of the proton in both NC and CC events. $Q^{2}$ is determined from the energy via $Q^{2}=2 m_{p} T_{p}$

An important background for this measurement is the misidentification of $\nu n \rightarrow$ $\nu n$ events as $\nu p \rightarrow-\nu p$. This background is particularly troublesome since the sensitivity of the $\nu n \rightarrow \nu n$ to $\Delta s$ has the opposite sign as compared to $\nu p \rightarrow \nu p$. Any misidentified $\nu n \rightarrow \nu n$ events dilute the sensitivity of $R_{N C / C C}$ to $\Delta s$. Much work has been done to keep this background low. It has been recently shown that the FINeSSE 
detector has good sensitivity to the $2.2 \mathrm{MeV}$ n-capture photon that is a signature of the $\nu n \rightarrow \nu n$. This has been exploited in the analysis with much success. The contamination from $\nu n \rightarrow \nu n$ is now quite low which has enabled a better measurement of $\Delta s$ than has been reported previously.

This strategy has been developed and tested using the GEANT3 Monte Carlo simulation of the detector and event reconstruction program as described above. A large sample of physics events with vertices evenly distributed within the nominal volume of the Vertex Detector $(2.5 \mathrm{~m})^{3}$ were tracked through the detector simulation. This event sample included both signal and background events as modeled by the NUANCE Monte Carlo [36]. The event types and relative frequencies in neutrino mode are shown in Table 3.1.

The event reconstruction cuts were optimized for high efficiency for the signal $\nu p \rightarrow \nu p$ and $\nu n \rightarrow \mu^{-} p$ events and large discrimination against background, especially $\nu n \rightarrow \nu n$. The identification of the n-capture $2.2 \mathrm{MeV}$ photon was particularly important in this. The results from this exercise are summarized in Table 5.2. Note the high purity and good efficiency for the signal $\nu p \rightarrow \nu p$ and $\nu n \rightarrow \mu^{-} p$ events.

\begin{tabular}{|r|rrrrr|}
\hline & \multicolumn{5}{|c|}{ reaction channel } \\
\hline NCp cuts & NCp & NCn & NC $\pi$ & CCQE & CC $\pi$ \\
\hline raw events & 39098 & 37544 & 35500 & 184032 & 100630 \\
passed events & 5668 & 483 & 131 & 203 & 24 \\
efficiency (\%) & 14.5 & 1.3 & 0.4 & 0.1 & 0.0 \\
fid. eff. (\%) & 21.3 & 1.9 & 0.5 & 0.2 & 0.0 \\
purity (\%) & 87.1 & 7.4 & 2.0 & 3.1 & 0.4 \\
\hline \hline CCQE cuts & NCp & NCn & NC $\pi$ & CCQE & $\mathrm{CC} \pi$ \\
\hline raw events & 39098 & 37544 & 35500 & 184032 & 100630 \\
passed events & 84 & 7 & 285 & 10090 & 1789 \\
efficiency (\%) & 0.2 & 0.0 & 0.8 & 5.5 & 1.8 \\
fid. eff. (\%) & 0.3 & 0.0 & 1.2 & 8.0 & 2.6 \\
purity (\%) & 0.7 & 0.1 & 2.3 & 82.0 & 14.5 \\
\hline
\end{tabular}

Table 5.2: Summary of events that passed the NCp and CCQE cuts along with efficiencies and purities: "efficiency" is the reconstruction efficiency throughout the $(2.5 \mathrm{~m})^{3}$ volume. "fid. eff." is the reconstruction efficiency within the $(2.2 \mathrm{~m})^{3}$ fiducial volume. The simulation data set contained $400 \mathrm{k}$ events. 
Using these reconstructed events, $G_{A}^{s}\left(Q^{2}=0\right)(=\Delta s)$, was calculated for the simulated neutrino data set and its sensitivity to various experimental errors was determined. The errors considered in this procedure include:

1. statistics of event samples;

2. neutrino backgrounds;

3. scattering from free protons in $\mathrm{CH}_{2}$;

4. experimental systematics in calculated efficiencies and $Q^{2}$ reconstruction;

5. and systematics due to uncertainties in backgrounds.

The resulting distributions from this procedure are summarized in Figure 5.11. As can be seen in this figure, the reconstructed event samples are sizable and the efficiency high in the $Q^{2}=0.2-0.6 \mathrm{GeV}^{2}$ range. The resulting measurement of $R_{N C / C C}$ is precise in this $Q^{2}$ range. In the $Q^{2}=0.25 \mathrm{GeV}^{2}$ bin, the simulated measurement of the ratio yields $R_{N C / C C}=0.148 \pm 0.004 \pm 0.006$ where the first error is statistical and the second is systematic.

This simulated measurement of $R_{N C / C C}$ over this range of $Q^{2}$ with the assumption of a dipole behavior of the strange axial form factor, $G_{A}^{s}$ (as discussed in Chapter 2), allows for an estimate of the error on $\Delta s$. It was determined to be 0.025 . This is a substantial reduction in error from previous work by our collaboration.

The sensitivity of this measurement to uncertainties in form factors has been estimated and is summarized in Figure 5.12. The dominant contributions to the form factor uncertainties come from the uncertainty in the axial vector mass, $M_{A}$, and from the isoscalar part of the vector form factor, $F_{2}^{s}$. The current world average of $M_{A}$ as measured in neutrino scattering is $1.00 \pm 0.02 \mathrm{GeV}[17,18]$. The sensitivity of $\Delta s$ on $F_{2}^{s}$ is weaker, but this form factor is not well known directly. The sensitivity to $F_{1}^{s}$ is negligible. The G0 experiment [31] will measure $F_{1}^{s}$ and $F_{2}^{s}$ to \pm 0.03 and \pm 0.09 respectively at $Q^{2}=0.3 \mathrm{GeV}^{2}$. These error estimates are used to determine the contribution from these form factors. Combining all these contributions in quadrature yields a contribution to the error on $\Delta s$ of \pm 0.02 from form factor uncertainties.

In summary, a neutrino measurement of $R_{N C / C C}$ will allow for an extraction of $\Delta s$ with an experimental error of \pm 0.025 and a systematic due to form factor uncertainties of \pm 0.02 . This is a precise measurement of $\Delta s$. 


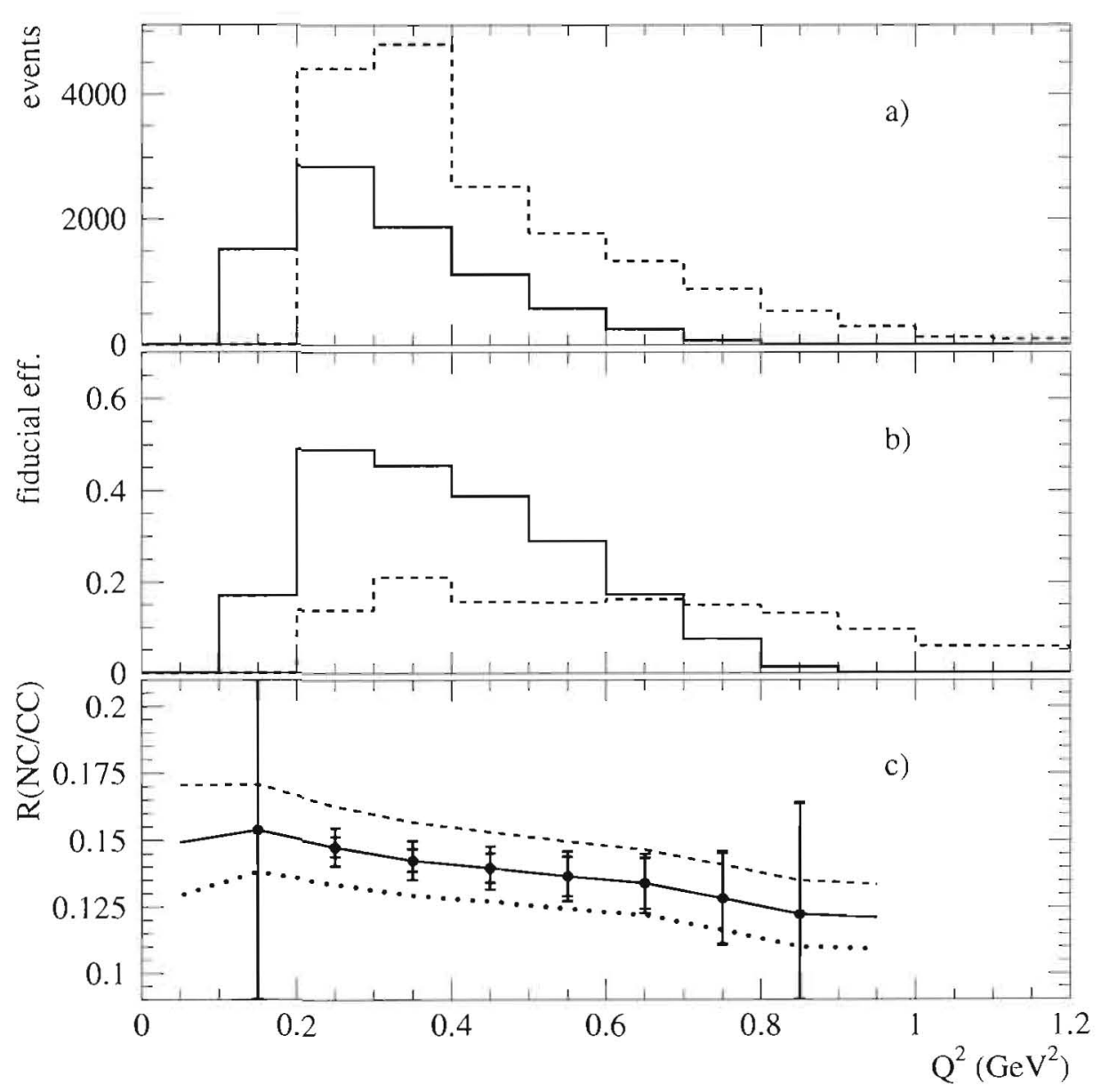

Figure 5.11: Results from the simulated neutrino measurement of $R_{N C / C C} \cdot Q^{2}$ distributions of (a) accepted $\nu p \rightarrow \nu p$ (solid) and $\nu n \rightarrow \mu^{-} p$ (dashed) events, (b) corresponding efficiencies within the fiducial volume, and (c) the reconstructed ratio, $R_{N C / C C}$. In (c) the simulated measurement is shown as data points with error bars (statistical and total error) along with the predicted values of $R_{N C / C C}$ at $\Delta s=0$ (solid line), -0.1 (dashed), and +0.1 (dotted). 

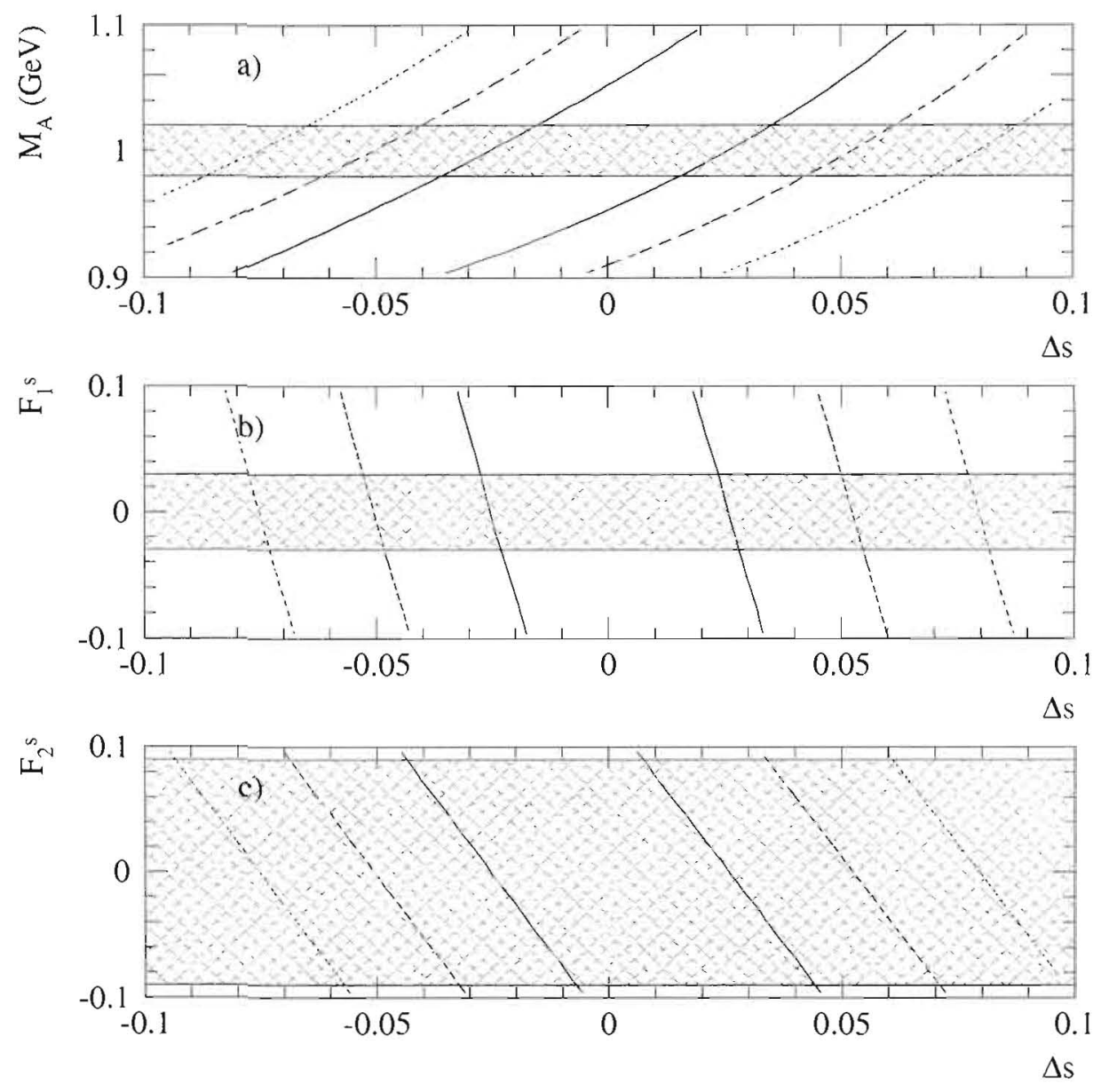

Figure 5.12: $\chi^{2}$ contours corresponding to $1 \sigma$ (solid), $2 \sigma$ (dashed), and $3 \sigma$ (dotted) resulting from the a fit procedure to extract $\Delta s$ from the simulated neutrino data set. The dependence of $\Delta s$ with (a) $M_{A}$, the axial vector mass, (b) $F_{1}^{s}$, and (c) $F_{2}^{s}$ is shown. The bands show the best current or future estimates of these parameters. 


\section{$\Delta s$ Measurement from Anti-neutrino Scattering}

To extract $\Delta s$ from the anti-neutrino data, the ratio,

$$
\bar{R}_{N C / C C}=\frac{\sigma\left(\overline{\nu_{\mu}} p \rightarrow \overline{\nu_{\mu}} p\right)}{\sigma\left(\overline{\nu_{\mu}} p \rightarrow \mu^{+} n\right)},
$$

will be used. This anti-neutrino ratio is actually more sensitive to $\Delta s$ than the analogous neutrino ratio, however, the measurement is more difficult for the following reasons.

- The flux and event rates are lower for anti-neutrinos. An anti-neutrino run time twice as long as the neutrino run will provide only half the total number of events.

- The anti-neutrino beam contains a substantial flux of neutrinos, so the actual ratio measured in anti-neutrino mode will be a combination of of $\bar{R}_{N C / C C}$ and $R_{N C / C C}$.

- The CC quasi-elastic channel for anti-neutrino scattering, $\overline{\nu_{\mu}} p \rightarrow \mu^{+} n$, does not have a proton in the final state, unlike in the neutrino case. This will cause a slightly higher systematic error.

Even with these slight disadvantages, a measurement of $\bar{R}_{N C / C C}$ is still possible, and, as seen below results is a respectable additional measurement of $\Delta s$.

A full simulation and reconstruction of anti-neutrino running has yet to be performed. That work will be done in the near future. However, based on the neutrino event reconstruction, an estimate of the error on $\Delta s$ resulting from an anti-neutrino measurement may be made. This exercise has been done. The major differences from the neutrino measurement are larger statistical errors and a larger systematic error due to the more difficult-to-reconstruct CC quasi-elastic anti-neutrino channel.

The resulting simulated measurement of $\bar{R}_{N C / C C}$ is shown in Figure 5.13. As noted, the errors (both statistical and systematic) are substantially larger than for the neutrino measurement. In the $Q^{2}=0.25 \mathrm{GeV}^{2}$ bin, the simulated measurement of the ratio yields $\bar{R}_{N C / C C}=0.181 \pm 0.006 \pm 0.008$ where the first error is statistical and the second is systematic. However, the extracted value for $\Delta s$ will be quite precise, due to the high sensitivity of $\bar{R}_{N C / C C}$ to $\Delta s$. The error on $\Delta s$, estimated from this procedure, is \pm 0.04 . The form factor uncertainties were estimated as they were for 


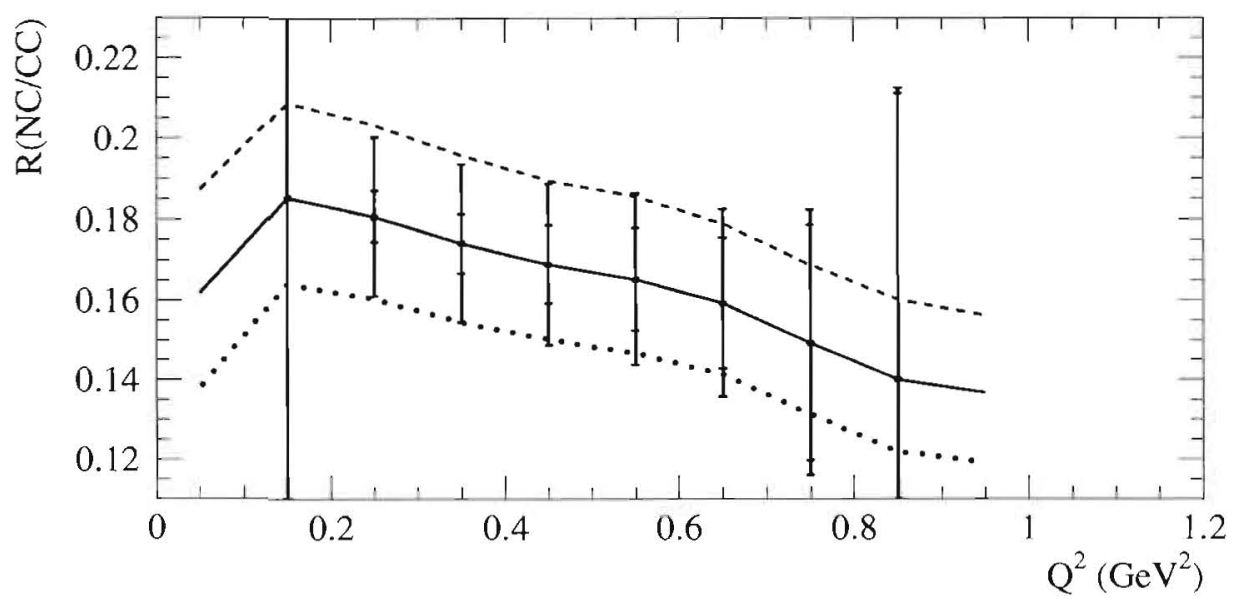

Figure 5.13: $Q^{2}$ distribution of the reconstructed ratio, $\bar{R}_{N C / C C}$, from the simulated anti-neutrino measurement. The simulated measurement is shown as data points with error bars (statistical and total error) along with the predicted values of $\bar{R}_{N C / C C}$ at $\Delta s=0$ (solid line), -0.1 (dashed), and +0.1 (dotted).

the neutrino measurement described above and are plotted in Figure 5.14. Note that the sensitivity of $\bar{R}_{N C / C C}$ to the $F_{2}^{s}$ is substantially smaller than for the neutrino measurement. This is a nice feature of the anti-neutrino measurement. Combining all contributions yields an error on $\Delta s$ of \pm 0.02 from form factor uncertainties.

In summary, an anti-neutrino measurement of $\bar{R}_{N C / C C}$ will allow for an extraction of $\Delta s$ with an experimental error of \pm 0.04 and a systematic due to form factor uncertainties of \pm 0.02 . This is a sufficiently precise measurement of $\Delta s$ and will be an important addition to the neutrino measurement. 

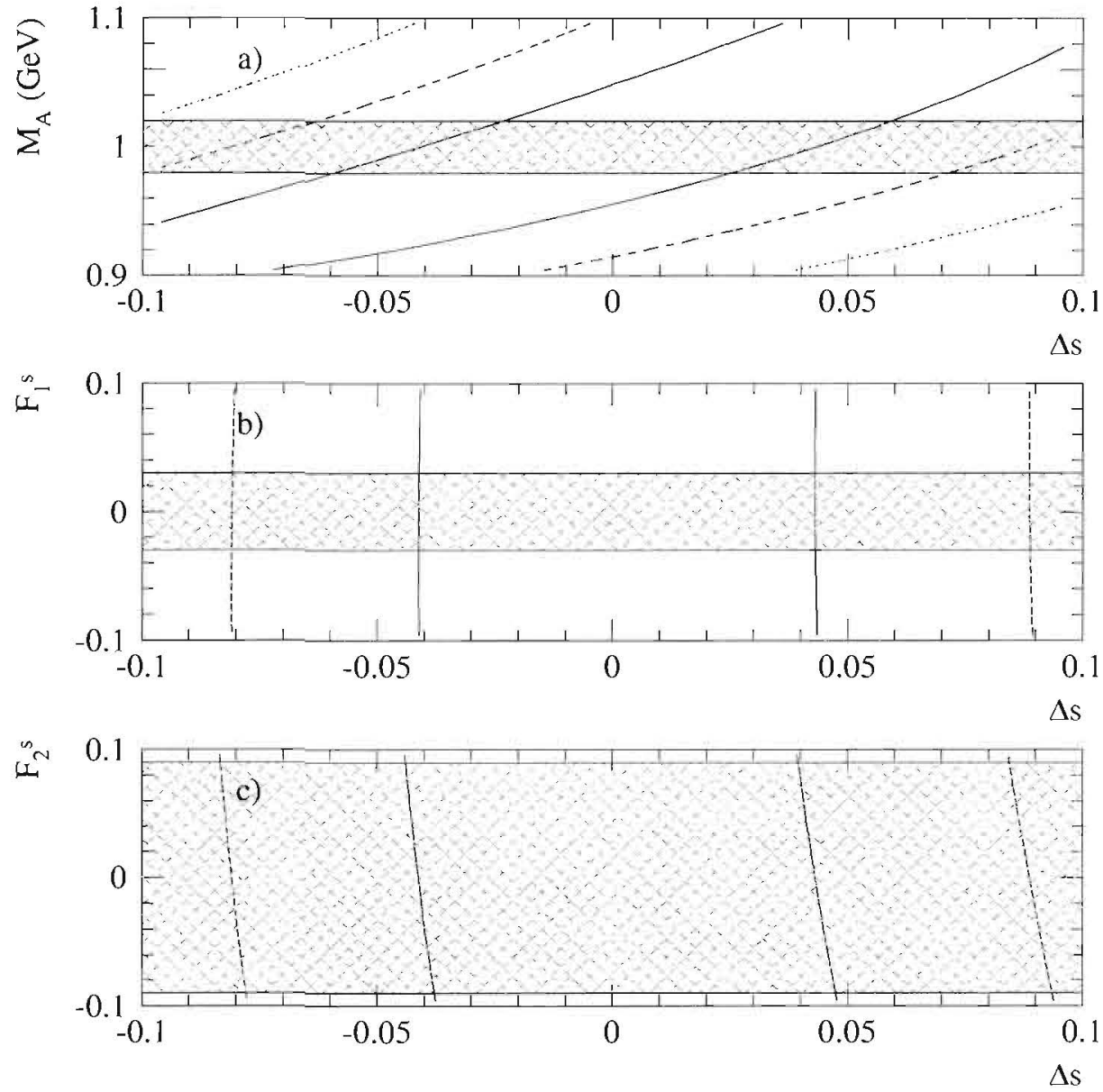

Figure 5.14: $\chi^{2}$ contours corresponding to $1 \sigma$ (solid), $2 \sigma$ (dashed), and $3 \sigma$ (dotted) resulting from the a fit procedure to extract $\Delta s$ from the simulated anti-neutrino data set. The dependence of $\Delta s$ with (a) $M_{A}$, the axial vector mass, (b) $F_{1}^{s}$, and (c) $F_{2}^{s}$ is shown. The bands show the best current or future estimates of these parameters. 


\subsubsection{Neutrino Cross Section Measurements}

As demonstrated in the preceding discussion, the CC QE and NC elastic channels can be easily reconstructed and isolated because of their simple event topologies. Making further use of the superior detector resolution and final state particle identification made available by this fine-grained detector technology, more complicated multi-track events can also be readily identified. This includes both resonant and coherent neutrino interactions producing a $\pi^{0}$ in the final state, as well as corresponding reactions in anti-neutrino mode running.

\section{Single Neutral Pion Measurements}

The superior event reconstruction capabilities of the proposed Vertex Detector will greatly enhance the ability to select $\pi^{0}$ interactions beyond that which is achievable in large open volume Cerenkov based detectors in present use. Neutral pions decay to two $\gamma \mathrm{s}$, which, in turn, pair produce leaving two clusters of charge. A $\nu_{\mu} p \rightarrow \nu_{\mu} p \pi^{0}$ interaction (Figure 5.15) can be distinguished by the presence of these two isolated energy deposition clusters corresponding to the converted $\gamma \mathrm{s}$ along with a short track from the final state proton. A $\nu_{\mu} n \rightarrow \nu_{\mu} n \pi^{0}$ interaction leaves just the energy deposition clusters from the converted $\pi^{0}$. NC coherent $\pi^{0}$ events (Figure 5.17) can be distinguished from the resonant events in this signal sample from the energies and angles of the two final state photons which can be used to determine if the $\pi^{0}$ angular distribution is more forward peaked as one expects for coherent scattering.

Over the course of the run proposed here, the experiment would accumulate a total sample of $\sim 23,000 \mathrm{NC} \pi^{0}$ resonant events and $\sim 6,500 \mathrm{NC}$ coherent $\pi^{0}$ interactions in neutrino mode. Reconstruction of the different cross section channels beyond what is used for the $\Delta s$ analysis is not yet complete. However a few very simple, pre-reconstruction cuts, show how powerful the "scibath" detection technique will be in identifying the different interaction channels. For example, with a few simple (pre-reconstruction) cuts described below, resonant and coherent neutral current single pion channels can be extracted with an efficiency of $34 \%$ and a purity of $54 \%$. As suggested from other fine-grained tracking detectors, reconstruction cuts will dramatically improve the purity level for this sample, already at the level of run-

ning experiments. The efficiency, already a factor of two better than open volume Čerenkov detectors, should also improve. 
The pre-reconstruction cuts applied to get a first-pass neutral pion sample include identifying clusters of charge in the $\mathrm{x}$ and $\mathrm{y}$ plane, cutting on visible energy in the sub-detectors, and identifying late light produced from muon decays at rest. It is anticipated that a full clustering algorithm in $\mathrm{x}, \mathrm{y}$, and $\mathrm{z}$, vertex position and direction reconstruction, and neutron tagging will greatly improve the purity of these samples.

Finally, charged current $\pi^{0}$ events such as $\nu_{\mu} n \rightarrow \mu^{-} p \pi^{0}$, (Figure 5.16) which are additionally accompanied by a final state muon track, can be tagged with some of the more sophisticated clustering algorithms under study. Similar efficiencies and purities are expected for anti-neutrino running.
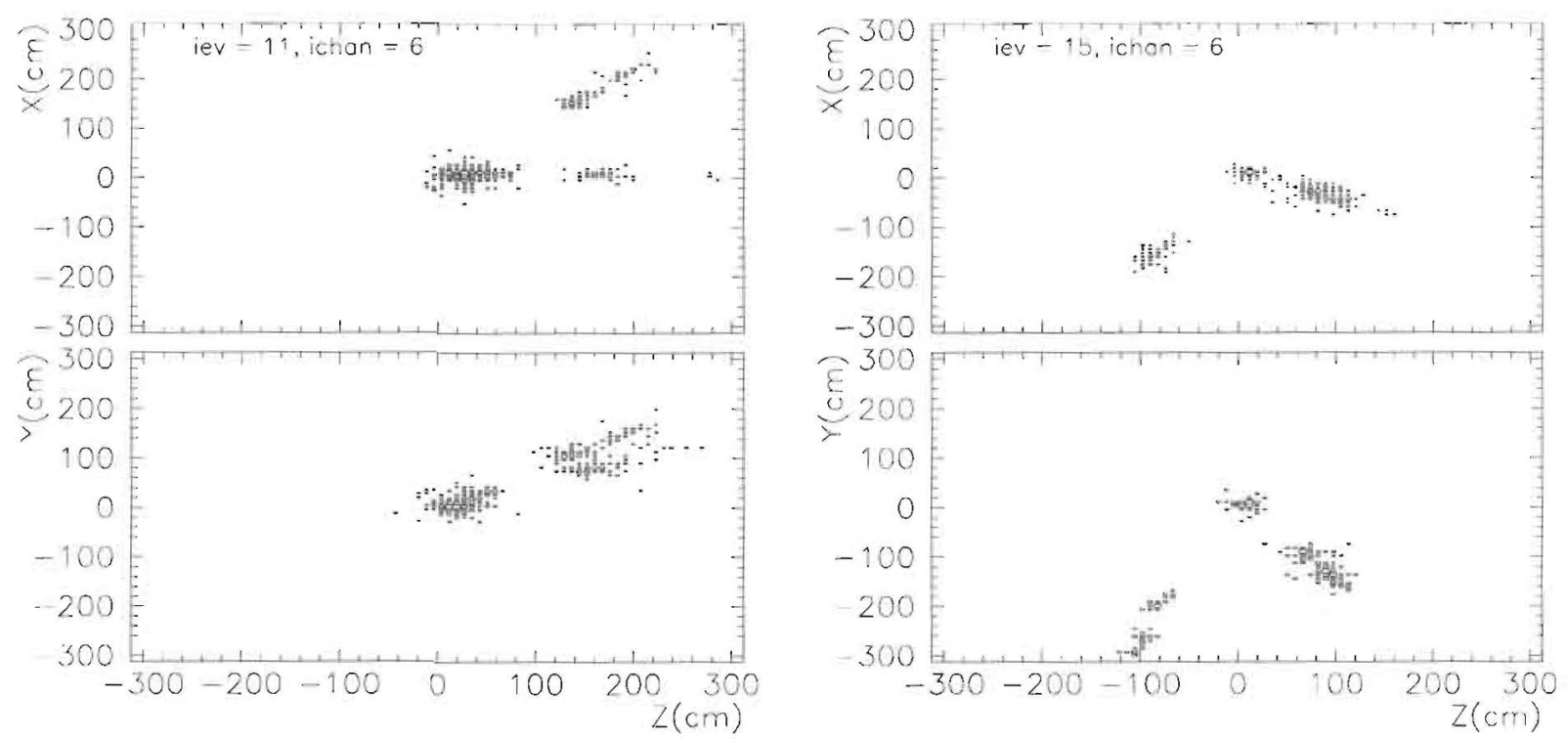

Figure 5.15: Hit-level simulations of $\mathrm{NC} \pi^{0}$ interactions in the Vertex Detector, $\nu_{\mu} p \rightarrow$ $\nu_{\mu} p \pi^{0}$. The three hit clusters correspond to the final state proton and two photons from $\pi^{0} \rightarrow \gamma \gamma$.

\section{Anti-neutrino Measurements}

Not only are low energy anti-neutrino cross section measurements desperately needed, but acquiring large samples of anti-neutrino data can provide additional information beyond what we've learned from neutrino scattering experiments. Here, we briefly describe two such examples: measurement of $\mathrm{NC} \pi^{0}$ and $\mathrm{CC} \pi^{-}$events in the antineutrino data. 

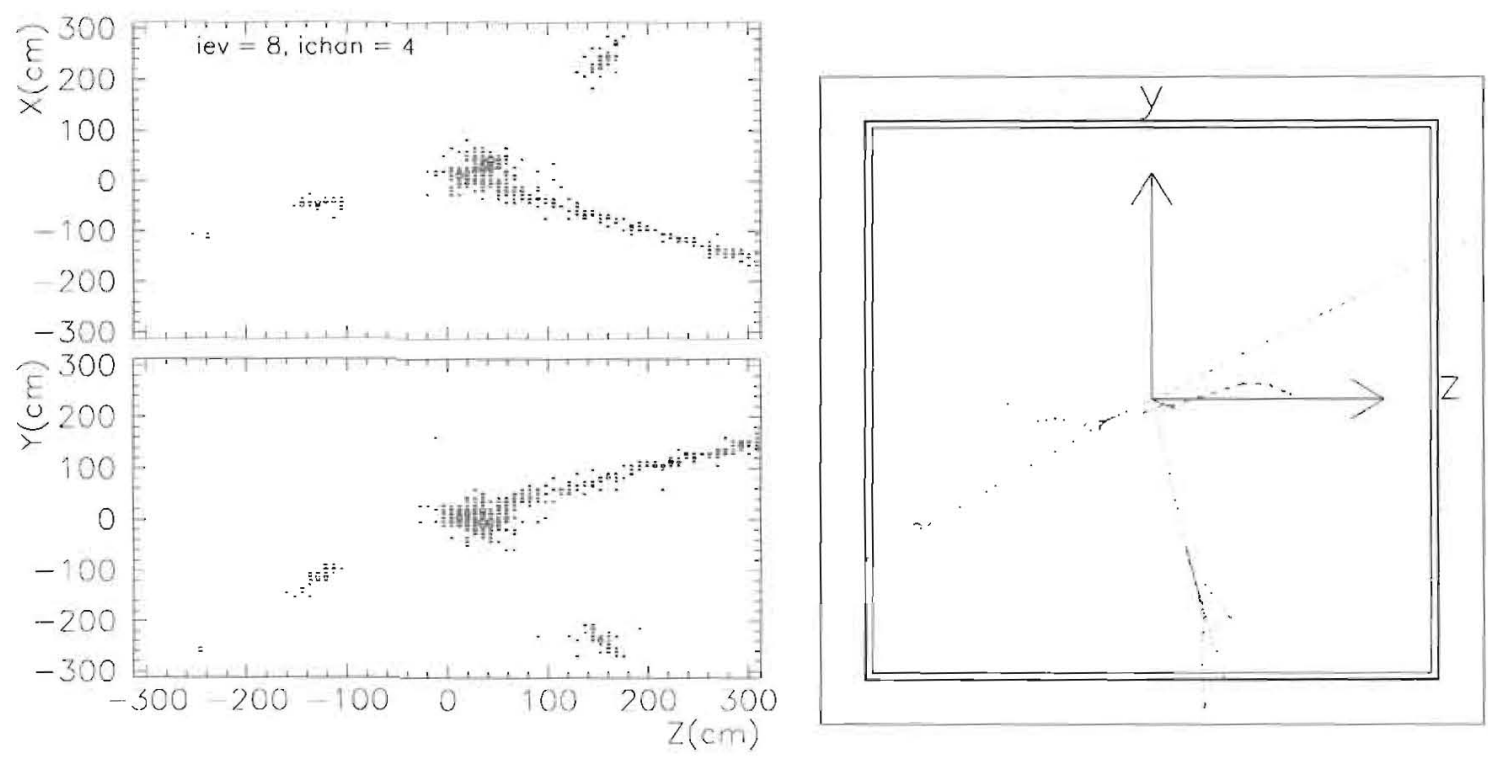

Figure 5.16: A CC $\pi^{0}$ interaction in the Vertex Detector, $\nu_{\mu} n \rightarrow \mu^{-} p \pi^{0}$ The right hand figure shows the true GEANT particle trajectories in the $Y Z$ plane. In this case, a muon is produced in addition to the three hit clusters from the proton and two photons.

Knowing the rate of anti-neutrino $\mathrm{NC} \pi^{0}$ backgrounds will be particularly important for future $\overline{\nu_{\mu}} \rightarrow \overline{\nu_{e}}$ oscillation searches. To date, there is only one published measurement of an anti-neutrino $\mathrm{NC} \pi^{0}$ production cross section which was reported with $25 \%$ accuracy by an early spark chamber experiment [38]. As we have already seen from Chapter 2 , the least well-known attribute of $\mathrm{NC} \pi^{0}$ production at low energy is the rate of coherent production. Anti-neutrino scattering can provide a unique and improved constraint on this particular mode of production. Because anti-neutrino resonant rates are expected to decrease (relative to neutrino mode rates), while the coherent rates remain the same [39], the coherent contribution is effectively amplified in the anti-neutrino data. Figure 5.18 contrasts the expected $\pi^{0}$ angular distributions for neutrino and anti-neutrino interactions. In the anti-neutrino case, the fraction of coherent $\pi^{0}$ events is double that in neutrino mode. The more dramatic excess of forward peaked events in the anti-neutrino data thereby allows a cleaner and more precise measurement of the coherent cross section.

In addition to NC $\pi^{0}$ cross section measurements that can be made in antineutrino running at FINeSSE, further exploration of CC resonant pion production rates is equally valuable. Because of the differing isospin content of the final states, the 

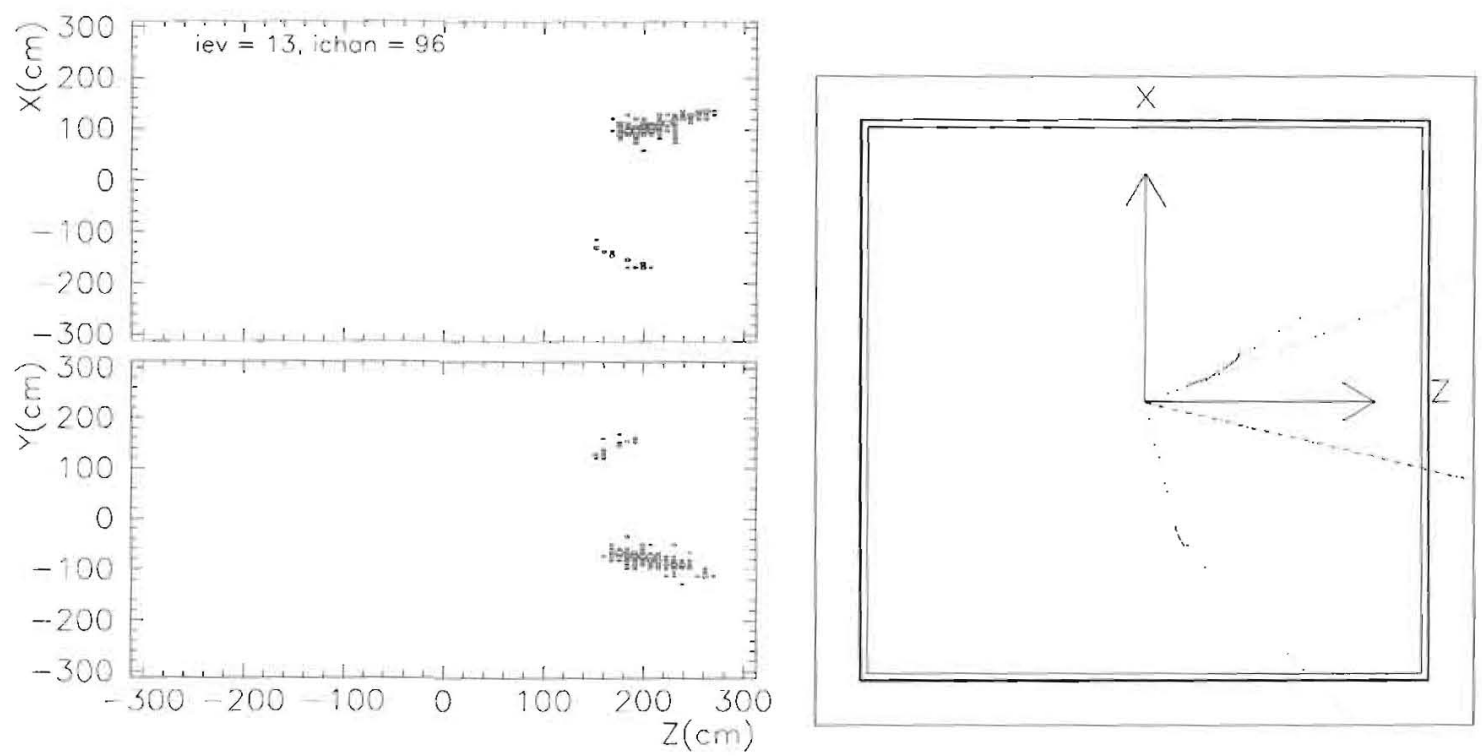

Figure 5.17: A NC coherent $\pi^{0}$ interaction in the Vertex Detector, $\nu_{\mu}{ }^{12} C \rightarrow \nu_{\mu}{ }^{12} C \pi^{0}$. The figure on the right shows the true GEANT particle trajectories in the $X Z$ plane for the same event. As can be seen, the hit clusters correspond to the two photons from the $\pi^{0}$ decay.

resonances produced in neutrino and anti-neutrino scattering are distinct. Measuring resonance production in anti-neutrino mode, and in particular reconstructing invariant mass distributions, allows one to probe these various resonant components. The ability to measure the reaction $\bar{\nu}_{\mu} p \rightarrow \mu^{+} p \pi^{-}$is particularly important as it is more sensitive to the effects of resonances beyond the $\Delta(1232)$, non-resonant backgrounds, and interferences between resonance and background amplitudes (Figure 5.19). Such effects have been only weakly constrained [62] by limited anti-neutrino statistics in this channel from decades old bubble chamber data [63]. Despite the fact that this data forms the basis of the models $[62,64]$ we employ in today's low energy neutrino Monte Carlo simulations, it is important to further test these fits with improved, higher statistics data. The fine-grained capabilities and anti-neutrino mode opportunities possible at FINeSSE could uniquely provide such a test. 

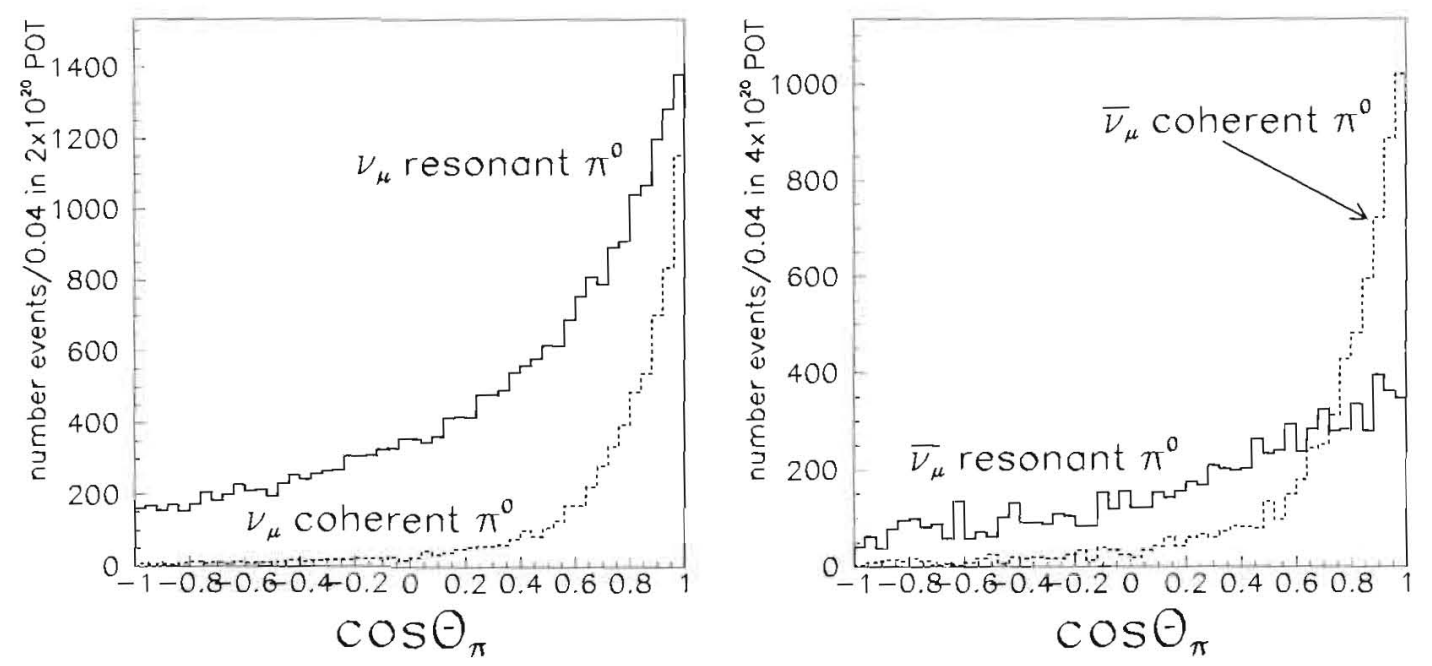

Figure 5.18: Generated $\pi^{0}$ angular distributions for resonant (solid) and coherent (dashed) NC $\pi^{0}$ interactions in neutrino (left) and anti-neutrino (right) running at FINeSSE. The coherently produced pions are more forward peaked in both cases.
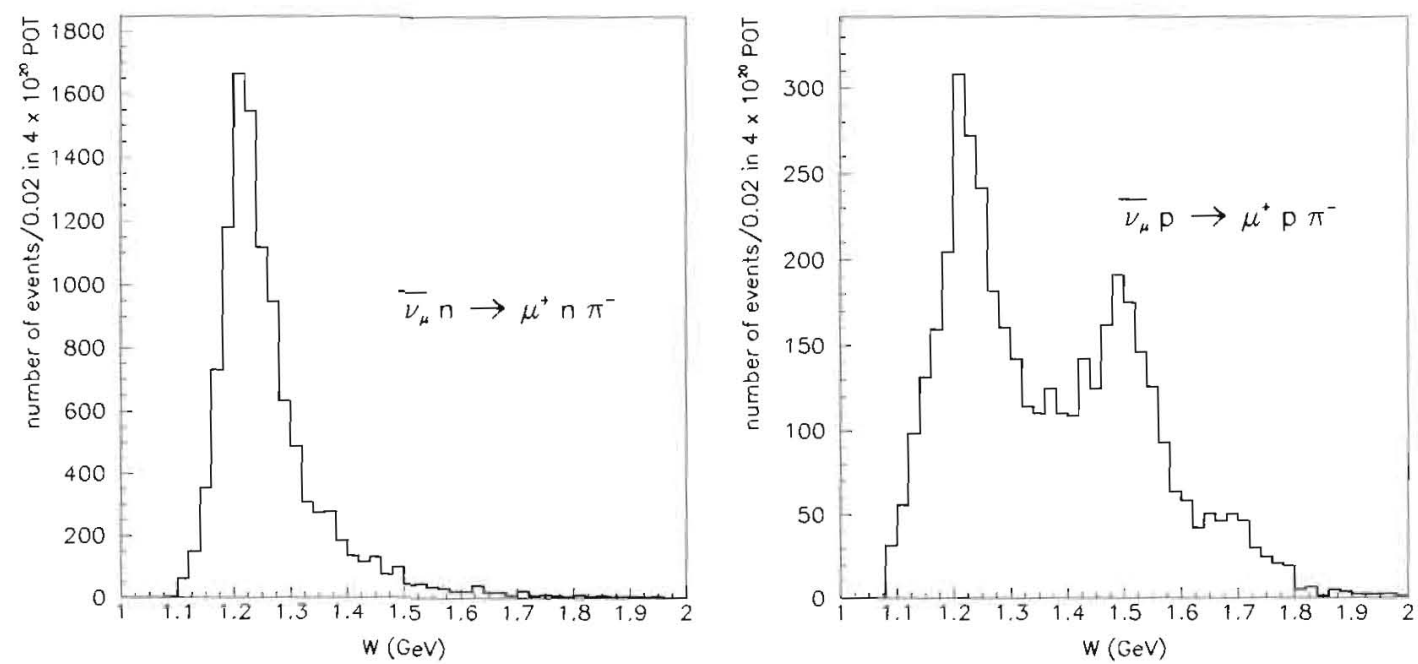

Figure 5.19: Generated invariant mass distributions for $\overline{\nu_{\mu}} \mathrm{CC}$ resonant $1 \pi^{-}$interactions at FINeSSE. The $\overline{\nu_{\mu}} n \rightarrow \mu^{+} n \pi^{-}$reaction (left) is dominated by the $\Delta(1232)$ resonance, whereas the $\overline{\nu_{\mu}} p \rightarrow \mu^{+} p \pi^{-}$reaction (right) clearly shows additional high mass contributions from resonances beyond the $\Delta(1232)$. 


\section{Chapter 6}

\section{Implementation at Fermilab}

This section gives the considerations for determining the location of the FINeSSE detector along the Booster Neutrino Beam Line.

The primary physics consideration for locating the detector is to maximize the Booster Neutrino Beam flux into the detector. The detector is to be centered on the beamline, and thus arl underground detector hall must be constructed. Also, the detector is to be located as close as possible to the target. There are two considerations which determine how close to the target the detector can be located.

The first consideration is the need to avoid disturbing the MiniBooNE Little Muon Counter (LMC) counting house during construction of the FINeSSE detector hall. Preliminary discussions with FESS indicate one should stay about 20 feet away from the existing counting house unless one wishes to employ more expensive excavation and construction techniques. An estimate of the location due to this consideration is no closer than 75.3 meters from the target.

The second consideration is the desire to keep the FINeSSE detector hall classified as "limited occupancy" according to the Fermilab Radiation Control Manual. This classification is to be in place during construction as well as during operation so that schedule coordination with beam operation is not required. The defining constraint is given in the MiniBooNE Shielding Assessment which conservatively requires 69.5 feet of soil to stop all muons created in the Booster Neutrino Beamline decay channel. A conservative estimate of the required location due to this consideration is 50 meters plus the 10 foot thickness of the 50 meter absorber plus the required soil, or no closer than 74.2 meters from the target. This estimate assumes the 25 meter absorber is 
not in place, since this is the configuration for FINeSSE. (If the 25 meter absorber were in place, the muons would stop short of the 74.2 meter line.)

The "80 meters from the target" location is an estimate for the upstream face of the detector. The $75.3 \mathrm{~m}$ and $74.2 \mathrm{~m}$ limitations given above relate to the upstream wall of the detector hall. The accurate location of the detector within the hall will be determined in the 2005 FINeSSE proposal and will include a proper update of the detector hall design from the November 2003 FINeSSE proposal, in which the upstream face of the detector was located at 100 meters from the target. Preliminary discussions with FESS indicate the cost of the detector hall would not change by moving it from 100 meters to 80 meters. The discussions included the need to change the orientation of the cover over the hall so that it moves transverse to the beam line rather than parallel to it. A detailed costing of the original proposed site $100 \mathrm{~m}$ from the target can be found in the FESS project definition report for the FINeSSE Detector [65].

The underground portion of the detector hall would very likely be classified as a confined space since it will only have one stairway for entrance or exit, again to save construction costs. The construction of the hall would have to be coordinated so that it does not disturb operations dependent on various infrastructure such as communication and utility ducts, some of which might serve MiniBooNE and NuMI, and eventually FINeSSE as well. 


\section{Chapter 7}

\section{Conclusions}

In this letter, we have put forth the case to build a small neutrino experiment at Fermilab to measure the strange-quark contribution to the nucleon spin as well as important neutrino and anti-neutrino interaction cross sections.

Determination of the strange spin of the proton, $\Delta s$, as described here, uses a theoretically robust method; intense, low-energy neutrino and anti-neutrino beams; and a novel detection technique.

Measurements of neutrino and anti-neutrino cross sections in this energy range are crucial for future oscillation measurements as well as for our understanding of these interactions. To further advance this knowledge, this experiment combines for the first time a high-statistics data sample with fine-grained detection capabilities.

This experiment can be mounted quickly and relatively inexpensively.

We look forward to comments and encouragement from the Fermilab PAC and Fermilab director regarding this Letter of Intent. Thank you for your consideration. 


\section{Bibliography}

[1] L. A. Ahrens et al., Phys. Rev. D35, 785 (1987).

[2] B. W. Filippone and X. D. Ji, Adv. Nucl. Phys. 26, 1 (2001), hep-ph/0101224.

[3] S. J. Brodsky et al., Phys. Lett. B206, 309 (1988).

[4] S. D. Bass, hep-ph/0311174; S. D. Bass and A. W. Thomas, hep-ph/9310306.

[5] S. L. Adler, Phys. Rev. 177, 2426 (1969); J. S. Bell and R. Jackiw, Nuovo Cimento 60A, 47 (1969).

[6] S.-L. Zhu et al., Phys. Rev. D66 034021 (2002).

[7] R. Buras, M. Rampp, H.-Th Janka, K. Kifonidis, PRL 90 (2003) 241101.

[8] C. J. Horowitz, PRD 65 (2002) 043001.

[9] J.R. Ellis, A. Ferstl and K.A. Olive, "Theoretical aspects of dark matter detection,", hep-ph/0106148.

[10] K. A. Olive, hep-ph/0308035.

[11] V. Barger et al., Phys. Rev. D65, 075022 (2002).

[12] B. Adeva et al., Phys. Lett. B412, 414 (1997).

[13] See, for example: V. A. Bednyakov, hep-ph/0310041.

[14] L. A. Ahrens et al., Phys. Rev. D34, 75 (1986).

[15] N. Baker et al., Phys. Rev. D23, 2499 (1982); K. Miller et al., ibid. 26, 537 (1982); T. Kitagaki et al., ibid. 28, 436 (1983). 
[16] G. Garvey et al., Phys. Rev. C48, 761 (1993).

[17] H. Budd, A. Bodek, and J. Arrington, hep-ex/0308005.

[18] E. Beise, nucl-ex/0501019.

[19] W. M. Alberico et al., Nucl. Phys. A651, 277 (1999).

[20] K. Ackerstaff et al. Nucl. Instrum. Meth. A 417, 230 (1998), hep-ex/9806008.

[21] A. Airapetian et al., Phys. Rev. D71, 012003 (2005).

[22] J. Pumplin et al., JHEP 0207, 012 (2002), hep-ph/0201195.

[23] M. J. Musolf at al., Phys. Rep. 239, 1 (1994).

[24] M. J. Musolf and B. R. Holstein, Phys. Lett. B242, 461 (1990).

[25] M. J. Musolf and T. W. Donnelly, Nucl. Phys. A546, 509 (1992).

[26] S.-L. Zhu et al., Phys. Rev. D66, 034021 (2002).

[27] D. T. Spayde et al., Phys. Lett. B583, 79 (2004); T. M. Ito et al., Phys. Rev. Lett. 92, 102003 (2004).

[28] The COMPASS Experiment, http://wwwcompass.cern.ch/, Alain Magnon and Gerhard Mallot, spokespersons.

[29] S. F. Pate, Phys. Rev. Lett. 92, 082002 (2004).

[30] K. A. Aniol et al., Phys. Lett. B509, 211 (2001).

[31] $G^{0}$ Experiment, http://www.npl.uiuc.edu/exp/Go/, Doug Beck, spokesperson.

[32] P. Lipari, Nucl. Phys. Proc. Suppl. 112, 274 (2002).

[33] A. Suzuki et al., Nucl. Instr. Meth. A453, 165 (2000); T. Ishii et al., Nucl. Instr. Meth. A482, 244 (2002).

[34] T. Ishida, Nucl. Proc. Suppl. 112, 132 (2002); C. Mauger, Nucl. Phys. Proc. Suppl. 112, 146 (2002); C. W. Walter, Nucl. Phys. Proc. Suppl. 112, 140 (2002).

[35] D. Rein and L. M. Sehgal, Annals Phys 133, 79 (1981). 
[36] D. Casper, Nucl. Phys. Proc. Suppl. 112, 161 (2002).

[37] E. Hawker, "Single Pion Production in Low Energy Neutrino-Carbon Interactions", NuInt02 workshop proceedings, to appear in Nucl. Proc. Suppl. (2004).

[38] H. Faissner et al., Phys. Lett. 125B, 230 (1983).

[39] D. Rein and L. M. Sehgal, Nucl. Phys. B223, 29 (1983).

[40] E. A. Paschos and A. V. Kartavtsev, hep-ph/0309148; J. Marteau et al.,hepph/9906449.

[41] J. Marteauat al., to be published in the proceedings of the 34th Recontres De Moriond: Electroweak Interactions and United Theories, hepph/9906449;E. A. Paschos at al., hep-ph/0309148; B. Z. Kopeliovich, Nucl. Proc. Suppl.139, 219 (2005), hep-ph/0409079.

[42] http://www.aps.org/

[43] A. A. Aguilar-Arevalo et al., "The MiniBooNE Run Plan", October 2003.

[44] http://wwwasd.web.cern.ch/wwwasd/geant4/geant4.html

[45] J. R. Sanford and C. L. Wang, BNL AGS internal report \# BNL11299 and \# BNL11479 (1967), and J. Monroe, "A Sanford-Wang Global Fit to Pion Production Data in the Momentum and Production Angle Range of Interest for the MiniBooNE Experiment", MiniBooNE Memorandum, September 2003.

[46] A. A. Aguilar-Arevalo et al.,"Addendum to the MiniBooNE Run Plan: MiniBooNE Physics in 2006"

[47] I. Chemakin et al., Phys. Rev. C65, 024904 (2002), nucl-ex/0108007.

[48] HARP collaboration, Status Report to SPSC, CERN-SPSC/2003-027, August 2003.

[49] M. Sorel, "Error on the MiniBooNE Neutrino Flux Arising from Finite HARP Statistics", MiniBooNE memo, August 2002.

[50] K. Nitta, et al., "The K2K Scibar Detector", hep-ex/0406023.

[51] http://usa.hamamatsu.com/. 
[52] IUCF Radiation Effects Research Program, http://www.iucf.indiana. edu/RERP.

[53] R. Brun, R. Hagelberg, M. Hansroul and J. C. Lassalle, "Geant: Simulation Program For Particle Physics Experiments. User Guide And Reference Manual," CERN-DD-78-2-REV.

[54] Saint-Gobain Crystals and Detectors, Newbury, OH 44065-9677, http://www.detectors.saint-gobain.com.

[55] Eljen Technology, Sweetwater, Texas 79556, http://www. eljentechnology.com.

[56] L. Bugel et al., "A Proposal for a Near Detector Experiment on the Booster Neutrino Beamline: FINeSSE: Fermilab Intense Neutrino Scattering Scintillator Experiment", Fall 2003.

[57] C.E. Allgower et al., Nucl. Instrum. Meth. A 499, 740 (2003).

[58] Arneodo et al., "Observation of long ionizing tracks with the ICARUS T600 first half-module," Nucl. Instrum. Meth. A 508, 287 (2003) [Erratum-ibid. A 516, $610(2004)]$.

[59] Flavio Cavanna, private communication.

[60] P. V. C. Hough, "Machine Analysis of Bubble Chamber Pictures", International Conference on High Energy Accelerators and Instrumentation, CERN, (1959).

[61] V. Bernard et al., J. Phys. G: Nucl. Part. Phys. 28 R1 (2002), hep-ph/107088.

[62] D. Rein, Z. Phys. C35,43 (1987).

[63] T. Bologneseet al.,P Phys. Lett.81B,393 (1979). S. J. Barish et al., Phys. Lett.91B,161 (1980). D. Allasia et al., Z. Phys.C20,95 (1983). P. Allen et al., Nucl. Phys. B264,221 (1986). H. J. Grabosch et al., Z. Phys.C41, 527 (1989).

[64] R. P. Feynmann et al., Phys. Rev.D3,2706 (1971).

[65] Project Definition Report: FINeSSE Detector, FESS/Engineering Project No.6$7-59$. 